\title{
Analytic surgery and the accumulation of eigenvalues
}

\author{
ANDREW HaSSEll ${ }^{b}$, RAFE MAZzeO ${ }^{\dagger}$ AND RichaRd B. Melrose ${ }^{\ddagger}$
}

Let $M$ be a compact manifold in which $H$ is an embedded hypersurface separating $M$ into two parts, $M_{+}$and $M_{-}$. If $h$ is a metric on $M$ and $x$ is a defining function for $H$ consider the family of metrics

$$
g_{\epsilon}=\frac{d x^{2}}{x^{2}+\epsilon^{2}}+h
$$

where $\epsilon>0$ is a parameter. The limiting metric, $g_{0}$, is an exact b-metric on the disjoint union $\bar{M}=M_{+} \cup M_{-}$, i.e. it gives $M_{ \pm}$ asymptotically cylindrical ends with cross-section $H$. In an earlier paper, [20], the behaviour of the Dirac operator associated to a Clifford module for the metric was analyzed as $\epsilon \downarrow 0$, and the limiting behaviour of the eta invariant, for $M$ odd-dimensional, was deduced, under the assumption that the induced Dirac operator $\partial_{H}$ on $H$ is invertible. It was shown that, modulo a $\mathbb{Z}$-valued term corresponding to the signature of small eigenvalues, the limit of $\eta\left(\partial_{\epsilon}\right)$ exists and is given by the regularized, 'b-eta' invariant of $\partial_{\bar{M}}$. In this second paper we study the same problem when $\partial_{H}$ has null space. The Dirac operator, or similarly the Laplacian, then has an infinite number of small eigenvalues, i.e. eigenvalues tending to zero with $\epsilon$. The central focus of this paper is the uniform analysis of the resolvent and heat kernel of generalized Laplacians as $\epsilon \downarrow 0$. As an application, we show that the limit of $\eta\left(\partial_{\epsilon}\right)$ is, again modulo a finite signature of small eigenvalues, the sum of $\eta\left(\partial_{\bar{M}}\right)$ and an extra term which is the eta invariant of a one-dimensional Dirac operator associated to the null space of $\partial_{H}$. This operator is determined by scattering data on $\bar{M}$ at zero energy, and controls both the leading behaviour of small eigenvalues as $\epsilon \downarrow 0$ and the long time asymptotics of the heat kernel.

This research was supported in part by NSF grant DMS-8907710 (b and $\ddagger$ ), by a Sloan Doctoral Dissertation Fellowship (b), NSF grant DMS-930326, an NYI Fellowship and a Sloan Foundation Fellowship $(\dagger)$ and and the Guggenheim Foundation $(\ddagger)$. 


\section{Introduction.}

\subsection{Analytic surgery.}

In this paper, we continue the study of analytic surgery initiated in [20]. By 'analytic surgery' we mean a singular deformation of a Riemannian metric on a closed manifold $M$ modeling 'surgery' in the sense of cutting $M$ along a hypersurface $H$ (possibly disconnected) and so forming a manifold with boundary $\bar{M}$. For simplicity we assume that $H$ separates $M$, so that $\bar{M}$ is the disjoint union of two manifolds with boundary $M_{ \pm}$. We consider a specific deformation which degenerates to a complete metric on $\bar{M}$ of the form $d x^{2} / x^{2}+h$, where $x$ is a boundary defining function for $H$ (that is, $x \geq 0, H=\{x=0\}$ and $d x \neq 0$ on $H$ ) and $h$ is a smooth metric on $M$. This form of metric on a manifold with boundary, called an 'exact b-metric' and studied in some detail in [22], gives $\bar{M}$ asymptotically cylindrical ends; $\log |x|$ is approximately the arc length parameter along each end. Specifically, we consider a metric family of the form

$$
g_{\epsilon}=\frac{d x^{2}}{x^{2}+\epsilon^{2}}+h ;
$$

this is a smooth metric on $M$ for every $\epsilon>0$, and as $\epsilon \rightarrow 0$ it develops an approximately cylindrical neck of length $2 \log 1 / \epsilon+O(1) \rightarrow \infty$. The singular limit at $\epsilon=0$ is an exact b-metric on $\bar{M}$.

Similar deformations, usually described as a family of manifolds with a long cylindrical neck across $H$ with length $l \rightarrow \infty$, have been studied by several authors. There are two main reasons for these studies. One is to understand the behaviour of geometric or topological invariants such as the index of a Dirac operator, eta invariant or analytic torsion under surgery, as in [6], [7], [8], [11], [26] and [12]. The other, more general reason, is to analyze the behaviour of the spectra of operators (such as the Laplacian) under the transition from closed manifold to complete manifold. In the first paper [20] and the present paper, both questions are investigated. Of course these two problems are closely related. In this paper a gluing formula for the eta invariant is presented. It is obtained by studying the full resolvent family of generalized Laplacians for metrics degenerating by surgery, and includes the analysis of accumulation of eigenvalues at the bottom of the continuous spectrum of $\Delta_{0}$. From an analytic perspective the main result of this paper is the precise description of the form of the 'degeneracy' of the resolvent family of the Laplacian (or Dirac operator) as the parameter $\epsilon \downarrow 0$. This is 
encoded in the description of the resolvent as an element of an algebra of pseudodifferential operators with properties tailored to the geometry of the degeneration.

Closely related work includes that of McDonald [21] and Seeley and Singer [28], who studied metric degeneration to incomplete conic metrics, and of Ji [17] and Wolpert [30], who studied degeneration of Riemann surfaces to surfaces with hyperbolic cusps. It should be remarked that the approach of McDonald inspired [20] and the present work. Gluing formulæ for the eta invariant have been discussed by several authors, in particular Bunke [6], Wojciechowski [29] and Brüning and Lesch [4]. We shall have more to say about these shortly.

There are good motivations for the choice of 'cylindrical ends' for $\bar{M}$ as the limit of the degeneration. In particular the well-known global boundary condition and index theorem for the Dirac operator on a manifold with boundary of Atiyah, Patodi and Singer, in [1], was obtained by attaching a cylindrical end to the boundary. The analytic properties of the Dirac operator and Laplacian in this case are well understood; a detailed analysis in the context of exact b-metrics can be found in [22]. There is a related construction, by Cheeger, where the boundary is 'coned off' to an incomplete conic metric. This was employed in [3], cf. also [9] and [10]. It would certainly be interesting to study metric degeneration to other types of complete metrics, such as metrics with asymptotically hyperbolic or Euclidean ends.

In a continuation of this paper, [14], the first-named author will present a surgery formula for analytic torsion with applications, including a combinatorial formula for 'b-analytic torsion' and a Hodge theoretic version of the Mayer-Vietoris sequence. In another direction, we shall use the result here to establish a signature theorem for manifolds with corners of codimension two, endowed with complete $b$-metrics [15].

\subsection{Eta invariant.}

The eta invariant was introduced by Atiyah-Patodi-Singer in [1] as the boundary term in their index formula for the Dirac operator on a manifold with boundary, with metric of product type near the boundary. (For a discussion of Dirac operators see [22], [2] or [18].) It is determined by the spectrum of the Dirac operator induced on the boundary, and is given by the analytic continuation of the eta function to $s=0$. Alternatively, and this is the formula we use below, it can be expressed in terms of the heat 
kernel:

$$
\eta(ð)=\frac{1}{\sqrt{\pi}} \int_{0}^{\infty} t^{\frac{1}{2}} \operatorname{Tr}\left(ð e^{-t \check{\varpi}^{2}}\right) \frac{d t}{t} .
$$

To obtain an index formula more general than that of [1], it is useful to extend the definition of the eta invariant to operators on complete manifolds. (There are other fruitful approaches, cf. [9], [10].) For manifolds with boundary with an exact b-metric, the Dirac operator has continuous spectrum, and the heat kernel is not trace class. Nevertheless, in [22], the b-eta invariant was defined as in (1.1), but with Tr replaced by the 'b-Trace' (see section 2.3); this is a natural regularization of the integral defining the trace of the heat kernel. The relation between the standard eta invariant and this regularized one is one of the themes of this paper. This b-eta invariant has also been studied by Müller [26] and Wojciechowski [29]; they establish a relation between it and the eta invariant for a manifold with a finite, but increasingly long, cylindrical end. Dai [11] also proves a result of this type, but only when the induced Dirac operator $\partial_{H}$ on the boundary is invertible, using the methods of [20].

The b-eta invariant and this regularization also shed light on possible extensions of the index theorem for Dirac operators on manifolds with corners endowed with complete b-metrics. One step in this direction is taken in [15].

\subsection{Statement of Results.}

Let us first recall the main results from the first paper [20], henceforth referred to as 'Part I'. There the same situation, for the eta invariant, was studied, under the assumption that the induced Dirac operator on $H$ is invertible. A 'surgery double space' and 'surgery heat space' were introduced as spaces to which the Schwartz kernels of $\left(\Delta-\lambda^{2}\right)^{-1}$ and $e^{-t \Delta_{\epsilon}}$ lift to be regular (i.e. conormal) uniformly as $\epsilon \rightarrow 0$. These spaces are blown up versions of $M^{2} \times\left[0, \epsilon_{0}\right]_{\epsilon}$ and $M^{2} \times[0, \infty]_{t} \times\left[0, \epsilon_{0}\right]_{\epsilon}$ which resolve the degeneracies of the space of vector fields $\mathcal{V}_{s}$ associated with the family $g_{\epsilon}$. The resolvent and heat kernel were shown to be polyhomogeneous conormal on these spaces, i.e. to have complete expansions in powers at all boundaries, and their leading asymptotics (model operators) at $\epsilon=0$ were explicitly identified. It was shown that the projector $\Pi_{\epsilon}$ onto eigenfunctions corresponding to eigenvalues tending to zero with $\epsilon$ is a finite rank, smoothing operator. These results led to the following result for the Dirac operator associated to an Hermitian Clifford module associated to the metric $g_{\epsilon}$, with 
unitary Clifford connection:

Theorem (Mazzeo-Melrose). If the induced Dirac operator on $H$ is invertible, then the eta invariant of $\check{\partial}_{\epsilon}$ satisfies

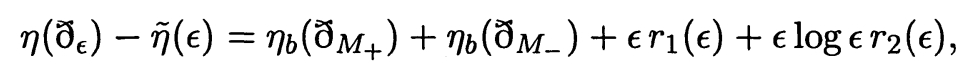

with $r_{i} \in C^{\infty}\left(\left[0, \epsilon_{0}\right]_{\epsilon}\right)$ and where $\tilde{\eta}(\epsilon)$ is the signature of $\Pi_{\epsilon}$.

Douglas and Wojciechowski [12] obtain similar results.

The assumption of invertibility of the operator induced on $H$ excludes many interesting cases. The main goal of this paper is to extend the machinery of Part I to include cases where the operator on $H$ has null space. To do so the constructions in Part I must be modified. One reason for this is that when $\Delta_{H}$ has null space the heat kernel no longer has uniform exponential decay, as it does (up to finite rank) in the circumstances of [20]. To calculate the integral (1.1) the leading behaviour of the heat kernel as $t \rightarrow \infty$ must be understood. This amounts to understanding the leading behaviour of the resolvent as $\lambda \rightarrow 0$. In contrast with Part I, the resolvent must therefore be analyzed near the bottom of the continuous spectrum of $\Delta_{0}$, and in particular, the leading behaviour of the small eigenvalues, those going to zero with $\epsilon$, must be discussed. To cope with this we perform further blowups on the spaces of Part I, to resolve singularities in the kernel which form upon approach to $\lambda=\epsilon=0$. We introduce the 'logarithmic double space' $X_{\mathrm{Ls}}^{2}$ and 'logarithmic heat space' $X_{\mathrm{LHs}}^{2}$; the names come from the 'logarithmic blowup' (see section 2.5) applied to each face of the surgery double space. On this space there are analogues of the results above. Consider the function ias $\epsilon=1 / \sinh ^{-1}(1 / \epsilon)$ ('inverse arc-sinh'), which is the reciprocal of the growth rate of the volume of $M$. It tends to zero with $\epsilon$, but only logarithmically. To understand the resolvent we rescale the spectral parameter $\lambda$ by setting $\lambda=($ ias $\epsilon) z$ to capture the behaviour of the small eigenvalues. When $\epsilon=0, \lambda=0$ for all $z$.

Theorem 1.1. The resolvent $\left(\Delta_{\epsilon}-(\text { ias } \epsilon)^{2} z^{2}\right)^{-1}$ is meromorphic as a family of half-densities on $X_{\mathrm{Ls}}^{2} \times \mathbb{C}$. The poles $z(\epsilon)$ have limits as $\epsilon \rightarrow 0$ equal to either $0, z_{j}$ or $\infty$, where the numbers $z_{j}^{2}$ are the eigenvalues of a onedimensional Laplacian $\mathrm{RN}(\Delta)$ on $[-1,1]$ with boundary conditions determined by scattering data on $\bar{M}$.

This is proved in section 6 . Indeed we extend the result in section 7 to the full resolvent which includes the resolvent away from the spectrum at $\epsilon=0$ 
as in Part I. From this we calculate the heat kernel by a contour integral. With $\Pi_{\epsilon}$ now denoting the (finite rank) projection onto eigenfunctions with 'very small' eigenvalues $\lambda^{2}(\epsilon)=o\left((\text { ias } \epsilon)^{2}\right)$, we have

Theorem 1.2. On $X_{\mathrm{LHs}}^{2}$, the projection of the heat kernel off the eigenmodes with very small eigenvalues, $e^{-t \Delta_{\epsilon}}\left(\mathrm{Id}-\Pi_{\epsilon}\right)$ is the product of $t^{-n / 2}$ with a smooth density for $t$ near zero and is smooth up to $t=\infty ; e^{-t \Delta_{\epsilon}} \Pi_{\epsilon}$ itself is smooth except possibly up to $t=\infty$.

In both cases we give the leading terms at each boundary face. In principle it is possible to calculate the Taylor series at every face at $\epsilon=0$ to arbitrarily high order. From these results the behaviour of the eta invariant can be read off.

Theorem 1.3. Let $\eta_{\mathrm{fd}}(\epsilon)$ be the signature of $\Pi_{\epsilon}$. Then

$$
\eta\left(\mathrm{\partial}_{\epsilon}\right)-\eta_{\mathrm{fd}}(\epsilon)=\eta_{b}\left(\mathrm{\partial}_{M_{+}}\right)+\eta_{b}\left(\mathrm{\partial}_{M_{-}}\right)+\eta\left(\mathrm{RN}\left(\mathrm{\partial}_{)}\right)+(\operatorname{ias} \epsilon) r(\operatorname{ias} \epsilon),\right.
$$

where $r$ is smooth.

Thus we get, in comparison with the case when $\partial_{H}$ is invertible, an extra contribution $\eta(\mathrm{RN}(\check{\delta}))$ coming from the very small eigenvalues. An explicit formula for this extra contribution in terms of the scattering matrix is given in proposition 5.2 .

This 'gluing formula' for the eta invariant may also be derived by combining the results of Bunke [6], [7] and Müller [26], at least for metrics which develop long product cylinders around $H$, for which the Dirac operators on $M, M_{+}$and $M_{-}$have no nullspace, and without the explicit estimate of the error term above.

Let us now describe this connection more precisely. Bunke considers two odd dimensional manifolds $M_{ \pm}$, with boundaries isometric to $H$ and $-H$, respectively. He forms the manifold $M$ by taking the union of these pieces with a cylinder $[-r, r] \times H$ joining the two pieces. His formula relates the eta invariant for the Dirac operator on the compact manifold $M$ with the eta invariants for the Dirac operators on the two manifolds with boundary $M_{ \pm}$. These latter two eta invariants are for the Dirac operators $\partial_{M_{ \pm}}$with certain self-adjoint boundary conditions on $H$. The usual Atiyah-PatodiSinger boundary operator would be the projector $\Pi_{-}$onto spinors over $H$ in the eigenspaces with negative eigenvalues for $\partial_{H}$; for the other boundary, $-H$, with Dirac operator $-\partial_{H}$, this should be replaced by the projector $\Pi_{+}=I-\Pi_{-}$. When the nullspace $\operatorname{Ker}\left(\widetilde{\partial}_{H}\right)$ is nontrivial, we choose Lagrangian subspaces $\Lambda_{ \pm} \subset\left(\partial_{H}\right)$ for the natural symplectic structure on this 
space. The boundary operators are then given by $\Pi_{\mp}+\Pi_{\Lambda_{ \pm}}$, where $\Pi_{\Lambda_{ \pm}}$ are the projections off $\Lambda_{ \pm}$. These give self-adjoint boundary conditions, and hence determine corresponding eta invariants $\eta\left(\partial_{M_{ \pm}}, \Lambda_{ \pm}\right)$.

There are additional correction terms in Bunke's formula. The most important of these is the eta invariant for the Dirac operator on $H \times[-r, r]$ with its product metric and with boundary conditions given by $\Pi_{-}+\Pi_{\Lambda_{ \pm}}$ at the two ends. This term is denoted $m\left(\Lambda_{+}, \Lambda_{-}\right)$. Bunke's formula then takes the form

$$
\begin{aligned}
& \eta\left(\check{\partial}_{M}\right)=\eta\left(\check{\partial}_{M_{+}}, \Lambda_{+}\right)+\eta\left(\check{\partial}_{M_{-}}, \Lambda_{-}\right)+m\left(\Lambda_{+}, \Lambda_{-}\right)+I\left(P_{+}, P_{-}\right)+\operatorname{dim} \operatorname{ker} ð_{M} \\
& -\operatorname{dim} \operatorname{ker}\left(\Im_{M_{+}}, \Lambda_{+}\right)-\operatorname{dim} \operatorname{ker}\left(\Im_{M_{-}}, \Lambda_{-}\right)+\operatorname{dim} \Lambda_{+} \cap \Lambda_{-} \text {. }
\end{aligned}
$$

Here $I\left(P_{+}, P_{-}\right)$is the index of a Fredholm pair of subspaces which arise in his construction.

To relate this formula to ours, we first use the result from [7]. This states that if the $\Lambda_{ \pm}$happen to correspond to the Lagrangians determined by the limiting values of solutions of $\partial_{M_{ \pm}}$(which we call $\Lambda_{ \pm}^{D}$ later), and it is assumed that $\Lambda_{+}$and $\Lambda_{-}$intersect only trivially and $\partial_{M}, \partial_{M_{ \pm}}$have only trivial nullspace, then for $r$ is sufficiently large, the relative index term $I\left(P_{+}, P_{-}\right)$vanishes. Furthermore, by the result of Müller [26], for these same special choices for $\Lambda_{ \pm}$, the eta invariants $\eta\left(\partial_{M_{ \pm}}, \Lambda_{ \pm}\right)$converge to the b-eta invariants $\eta_{b}\left(\widetilde{\partial}_{M_{ \pm}}\right)$. However, with these hypotheses, the finite dimensional term $\eta_{\mathrm{fd}}(\epsilon)$ in our formula is also trivial. Finally, the final term $m\left(\Lambda_{+}, \Lambda_{-}\right)$ agrees with our term $\eta(\mathrm{RN}(\widetilde{\delta}))$. Thus the formulas correspond in this special case.

\subsection{Outline of the proof.}

We may take as starting point for the considerations in this paper the fact that the eigenfunctions with eigenvalues tending to zero in the surgery limit are not always smooth on the 'single surgery space'

$$
X_{s}=\left[M \times\left[0, \epsilon_{0}\right]_{\epsilon} ; H \times\{0\}\right]
$$

of Part I; indeed they are not even continuous. One can easily see this in the case of surgery on an interval, or circle. Then the eigenfunctions of $\Delta_{\epsilon}$ are of the form $e^{2 \pi i k r / L_{\epsilon}}$, where $r=\sinh ^{-1}(x / \epsilon)$ is the arclength and $2 L_{\epsilon}$ is the length of the interval or circle with respect to $g_{\epsilon}$. On $X_{s}$, this eigenfunction is equal to 1 on one of the boundaries at $\epsilon=0$ (the lift of $H \times\{0\})$ and $(-1)^{k}$ on another adjacent boundary. The oscillations disappear into the intersection of these faces. To resolve these oscillations 
we replace, in section 3 , the single space $X_{s}$ by a new space, $X_{\mathrm{Ls}}$, on which the scaled distance $r / L_{\epsilon}$ is a smooth function. $X_{\mathrm{Ls}}$ is a blown up version of $X_{s}$ involving the operation of logarithmic blowup described in section 2.5. We then modify, in a methodical way, all constructions in Part I to reflect this change. The Lie algebra $\mathcal{V}_{s}$ of Part I lifts to $\mathcal{V}_{\mathrm{Ls}}$ on $X_{\mathrm{Ls}}$. The properties of $\mathcal{V}_{\mathrm{LS}}$, including its normal operators, are discussed in section 3.

We microlocalize the Lie algebra $\mathcal{V}_{\mathrm{Ls}}$ according to the general principles set forth in [25]. This means we construct the following objects. First, we need a double space $X_{\mathrm{Ls}}^{2}$ to carry Schwartz kernels of 'logarithmic surgery pseudodifferential operators', or Ls- $\psi$ dos. The diagonal submanifold $\Delta_{\text {Ls }}$ of $X_{\mathrm{Ls}}^{2}$ is such that kernels of $\mathcal{V}_{\mathrm{Ls}}$-differential operators are identified precisely with all distributions on $X_{\mathrm{Ls}}^{2}$ supported on $\Delta_{\mathrm{Ls}}$ and smooth along it. The Fourier transform in directions transverse to $\Delta_{\mathrm{Ls}}$ of these distributions are therefore polynomials. By replacing polynomial symbols with arbitrary classical symbols, we obtain the 'small calculus'. The double space has natural maps down to the single space $X_{\mathrm{Ls}}$ which are b-fibrations (see section 2); this means that the kernels induce operators on appropriate space of conormal distributions on $X_{\mathrm{Ls}}$. Thirdly, there is a triple space $X_{\mathrm{Ls}}^{3}$ with b-fibrations to $X_{\mathrm{Ls}}^{2}$; this is used to analyze to composition properties of Ls- $\psi$ dos. Finally, the normal operators on $\mathcal{V}_{\mathrm{Ls}}$ extend to 'model operator maps' on Ls- $\psi$ dos given by restriction of the kernel to faces at $\epsilon=0$. Operators, such as the resolvent family, are constructed by taking as parametrices elements of the operator calculus with appropriate model operators and then using iteration in the calculus to remove the errors. Geometric lemmas to help construct the spaces are given in the second half of section 2 and the surgery pseudodifferential calculus is set up in section 4 .

In view of the Pushforward theorem of [24], discussed here in section 2.3, the fact that there are b-fibrations $X_{\mathrm{Ls}}^{3} \rightarrow X_{\mathrm{Ls}}^{2} \rightarrow X_{\mathrm{Ls}}$ means that we can work within the class of polyhomogeneous conormal functions throughout. This has the virtue of almost eliminating estimates from the analysis, as it is easy to read off the decay rate of a function from the index set specifying its (poly)homogeneities. This comes at the cost of fairly complicated spaces and geometric machinery but we are able to obtain detailed information about the resolvent, heat kernel and small eigenvalues with this method. Because of the logarithmic blowups we are able to work with 'integral' index sets (see section 2.3) and show that our final objects, the resolvent, heat kernel and eta invariant, are smooth in the blown-up coordinates.

In section 5 we analyze the model problem coming from the reduced normal operator introduced in section 3. This is a new model operator, not appearing in Part I, and Proposition 3.8 indicates that the eigenvalues 
of this model control the leading behaviour of small eigenvalues of $\Delta_{\epsilon}$. We show that the resolvent of this model problem is well-behaved on the double space; this indicates that $X_{\mathrm{Ls}}^{2}$ is the 'correct' space to use; the resolvent of the model is used heavily in section 6 in the construction of the actual resolvent.

In sections 6 and 7 the problem of analyzing $\left(\Delta_{\epsilon}-\lambda^{2}\right)^{-1}$ as $\lambda$ approaches the spectrum is attacked. We construct the resolvent near the bottom of the continuous spectrum, 0 . To do so submanifolds at $\lambda=0$ are blown up, introducing the rescaled parameter $z=\lambda \sinh ^{-1}(1 / \epsilon)$ which captures the scaling of the small eigenvalues. To construct a parametrix we need to solve not only for the symbol at the diagonal singularity but also solve a number of model problems at each boundary face. Compatibility conditions at the intersections of faces give boundary conditions for these model operators, which enable them to be solved uniquely; the interaction between the models on different faces and of different orders is fairly complicated.

Once the resolvent is shown to be an element of the appropriate calculus the Schwartz kernel of a function of the Laplacian can be analyzed using the functional calculus. We construct the heat kernel in this way in section 8 . More precisely, we obtain it by performing the contour integral

$$
e^{-t \Delta_{\epsilon}}=\frac{i}{2 \pi} \int_{\Gamma} e^{-t \lambda^{2}}\left(\Delta_{\epsilon}-\lambda^{2}\right)^{-1} 2 \lambda d \lambda
$$

Then we obtain the eta invariant by performing the integral (1.1). This integral is more correctly to be thought of as a pushforward, since the integrand is defined on a blown-up version of its space of parameters. We construct spaces so that the integral becomes a pushforward under a b-fibration. This allows us to conclude that the result is polyhomogeneous, and a simple extra argument shows that it is actually smooth. We compute the leading terms of the heat kernel at $t=\infty$. Finally, in section 9 we apply this machinery to the eta invariant, obtaining Theorem 1.3.

\section{Manifolds with corners, blowups and b-fibrations.}

In this section we present material on the geometry of manifolds with corners that is needed in this paper. We will assume some familiarity with [24], but also recall some of this material in the first three subsections, sometimes with slightly different presentation. We then go on to describe two 'new' blowup operations, of logarithmic blowup and total boundary blowup, which are used heavily in the sequel. 


\subsection{Manifolds with corners.}

We refer to [24] for a discussion of manifolds with corners, the b-tangent space, b-maps, and b-fibrations. The set of boundary hypersurfaces of a manifold with corners $X$ is denoted $M_{1}(X)$, the set of all boundary faces is denoted $M(X)$ and the set of proper faces, that is excluding only $X$ itself, is denoted $M^{\prime}(X)$. A boundary defining function $\rho$ for a boundary hypersurface $H$ of a manifold with corners $Y$ is a smooth nonnegative function on $Y$ such that $H=\{\rho=0\}$ and $d \rho \neq 0$ on $H$. A smooth map between manifolds with corners $f: X \rightarrow Y$ is an (interior) b-map if for every boundary defining function $\rho_{H}$ for an element $M \in M_{1}(Y)$,

$$
f^{*} \rho_{H}=a \cdot \prod_{G \in M_{1}(X)} \rho_{G}^{e_{f}(G, H)}, 0<a \in C^{\infty}(X)
$$

where the (uniquely determined) collection of natural numbers $e_{f}(G, H)$, are the 'boundary exponents' of $f$. A total boundary defining function for $X$ is the product of boundary defining functions for all the boundary hypersurfaces

$$
\rho_{Y}=\prod_{H \in M_{1}(Y)} \rho_{H} .
$$

We will be particuarly interested in the special b-maps called bfibrations. The map $f$ above is a b-fibration if $f_{*}$, acting on the b-tangent bundle, is surjective on each fibre, and the image of each boundary hypersurface in $X$ is either $Y$ or one boundary hypersurface $H \subset Y$. (This definition is different from, but equivalent to, the definition given in [24].) Such bfibrations have good mapping properties on $M(X)$; the image of any face $F \in M(X)$ is a face in $M(Y)$, and $f\lceil F$ is a b-fibration onto its image.

Most of the b-fibrations, $f: X \longrightarrow Y$, we consider below have an additional property, namely

$$
f^{*} \rho_{Y}=a \cdot \rho_{X}, \quad 0<a \in C^{\infty}(X) .
$$

In terms of the boundary exponents this amounts to requiring that $e_{f}(G, H)$ $=0$ or 1 for each $G \in M_{1}(X)$ and $H \in M_{1}(Y)$ and that for each $G \in M_{1}(X)$ there exists precisely one $H \in M_{1}(Y)$ with $e_{f}(G, H)=1$. The assumption that $f$ is a b-fibration means that there can be at most one such $H$ for each G.

Definition 1. We say that a b-fibration is simple if it satisfies (2.2). 
p-submanifolds There are various possible definitions of a submanifold in a manifold with corners; we will use a very strong definition.

A subset $S \subset Y$ is a p-submanifold if locally, in some coordinate system $x_{1}^{\prime}, \ldots, x_{k^{\prime}}^{\prime}, y_{1}^{\prime}, \ldots, y_{n^{\prime}-k^{\prime}}^{\prime}$, with $x_{i}^{\prime} \geq 0$, and $y_{j}^{\prime} \in(-\delta, \delta), S$ is given by the vanishing of some subset of them:

$$
S=\left\{x_{i_{1}}^{\prime}=\ldots x_{i_{l^{\prime}}}^{\prime}=y_{j_{1}}^{\prime}=\cdots=y_{j_{m^{\prime}}}^{\prime}=0\right\} .
$$

Then if $S$ is connected it has a tubular neighbourhood which is a bundle over $S$, the fibre being a neighbourhood of $0 \in \mathbb{R}_{+}^{k^{\prime}-l^{\prime}} \times \mathbb{R}^{n^{\prime}-k^{\prime}-m^{\prime}}$. We say $S$ is an interior p-submanifold if $l^{\prime}=0$ in this definition. If $f$ is a b-fibration and $S$ is an interior p-submanifold then $f^{-1} S$ is a p-submanifold. If $S$ is not interior, then in general $f^{-1} S$ is a union of p-submanifolds of $X$. This can be seen in local coordinates as follows. If coordinates in $Y$ are chosen so that $S$ has the form above it is then possible to choose coordinates $x_{1}, \ldots x_{k}, y_{1}, \ldots y_{n-k}$, in $X$ with $x_{i} \geq 0, y_{j} \in(-\delta, \delta)$ so that locally $f$ has the form

$$
f\left(x_{1}, \ldots x_{k}, y_{1}, \ldots, y_{n-k}\right)=\left(\prod_{r \in I_{1}} x_{r}, \cdots \prod_{r \in I_{k^{\prime}}} x_{r}, y_{1}, \ldots y_{n^{\prime}-k^{\prime}}\right) .
$$

Then $f^{-1} S$ is locally a union of the p-submanifolds $\left\{x_{r_{1}}=\cdots=x_{r_{k^{\prime}}}=\right.$ $\left.y_{j_{1}}=\cdots=y_{j_{m^{\prime}}}=0\right\}$, with $r_{i} \in I_{i}$.

Degrees and density bundles It is convenient to introduce the notion of the 'degree' of a boundary hypersurface $H$ of a manifold with corners $X$. This is simply an assignment of an integer, $d(H)$, to $H$. Such an assignment to each boundary hyersurface of a manifold with corners, $d: M_{1}(X) \longrightarrow \mathbb{Z}$, is intended to indicate the 'basic' order of growth of densities allowed at the boundary hypersurfaces $H$. The degree density bundle is defined by

$$
\Omega_{D}(X)=\prod_{H \in M_{1}(X)} \rho_{H}^{-d(H)} \Omega_{b}(X)=\prod_{H \in M_{1}(X)} \rho_{H}^{-d(H)-1} \Omega(X) .
$$

In general $d$ is fixed once and for all for a given manifold with corners. Observe that $b$-densities, corresponding to all degrees being zero, have the pleasant property that $d \rho_{H} / \rho_{H}$ is a canonical factor at $H$, so dividing by $\left|d \rho_{H} / \rho_{H}\right|$ gives a canonical identification of the restriction of $\Omega_{b}(X)$ to $H$ and $\Omega_{b}(H)$. With general $D$-densities, restriction defined by division by $\left|d \rho_{H} / \rho_{H}^{d(H)+1}\right|$ depends on the choice of boundary defining function. However, in this paper we will have a canonical total boundary defining function $R$ for many of our spaces. When this is so, if the degrees of boundary hypersurfaces $H \cap K\left(K \in M_{1}(X)\right)$ of $H$ are defined by $d(H \cap K)=d(K)-d(H)$, 
then division by $\left|R^{-d(H)} d \rho_{H} / \rho_{H}\right|$ gives a canonical restriction

$$
C^{\infty}\left(X ; \Omega_{D}(X)\right) \rightarrow C^{\infty}\left(H ; \Omega_{D}(H)\right)
$$

\subsection{Blowups.}

If $S \subset Y$ is a p-submanifold, the blowup of $Y$ at $S$, denoted $[Y ; S]$, is a manifold with corners, given as a point set by $(Y \backslash S) \cup\left(\mathrm{SN}^{+} S\right)$, the union of the complement of $S$ in $Y$ with the (inward-pointing) unit sphere bundle over $S$, and with the unique minimal $C^{\infty}$ structure such that the lifts of $C^{\infty}$ functions on $Y$ and polar coordinates at $S$ are smooth. There is a unique smooth map $[Y ; S] \rightarrow Y$ extending the identity on $Y \backslash S$, called the blowdown map. The lift of a p-submanifold $T \subset Y$ to $[Y ; S]$ is defined if (i) $T \subset S$, in which case the lift is defined to be the inverse image of $T$ under the blowdown map or (ii) $T \backslash S$ is dense in $T$, in which case the lift is defined as the closure of $T \backslash S$ in $[Y ; S]$. In either of these cases we can define an iterated blow-up $[Y ; S ; T]=[[Y ; S] ; T]$ where, by an abuse of notation, the $T$ on the right is actually the lift of $T$ to $[Y ; S]$, and is by implication a p-submanifold.

We will often perform sequences of blowups to create new spaces in this paper, and it is important to know when one can exchange the order of blowup. We use the following elementary result.

Lemma 2.1. If $S, T$ are p-submanifolds of $Y$ and either (i) $S$ and $T$ are transverse or (ii) $T \subset S$ then $[Y ; S ; T]=[Y ; T ; S]$.

Proof. The proof of (i) is immediate because then $N S$ and $N T$ are independent and so the blowups occur in two disjoint sets of variables.

To prove (ii), it is sufficient to consider the case $T=\{0\}, S=\mathbb{R}_{+}^{l} \times \mathbb{R}^{m}$, $Y=\mathbb{R}_{+}^{k} \times \mathbb{R}^{n}, k \geq l, n \geq m$. Define $R_{T}^{2}=\sum_{i=1}^{k} x_{i}^{2}+\sum_{j=1}^{n} y_{j}^{2}$ and $R_{S}^{2}=$ $\sum_{i=l+1}^{k} x_{i}^{2}+\sum_{j=m+1}^{n} y_{j}^{2}$. In both $[Y ; S ; T]$ and $[Y ; T ; S]$ the lift of $T$ and $S$ have boundary defining functions (the lifts of) $R_{T}$ and $R_{S}$ respectively, and a superset of coordinates on the lift of $T$ is given by $x_{i} /\left(x_{i}+R_{T}\right)$, $y_{j} /\left(y_{j}+R_{T}\right)$ for $i, j \geq 1$ and on the lift of $S$ by $x_{i} /\left(x_{i}+R_{S}\right), y_{j} /\left(y_{j}+R_{S}\right)$ for $i \geq l+1, j \geq m+1$ on both spaces. This means that the identity map on $Y \backslash S$ extends to a smooth map $[Y ; S ; T] \leftrightarrow[Y ; T ; S]$ in both directions. 
Each extension is therefore a canonical diffeomorphism.

\subsection{Operations on conormal functions.}

The principal function spaces used in this paper are spaces of polyhomogeneous conormal functions, conormal either at a boundary hypersurface or an interior p-submanifold (e.g., the diagonal). We refer the reader to [24] for the definitions of and notation for multiweights, index sets and families and of various spaces of conormal distributions.

In this paper we will mostly work with special types of polyhomogeneous distributions which are close to being smooth:

Definition 2. An index set $E$ is natural (respectively integral) if all its powers are natural numbers (resp. integers), that is, $(z, k) \in E \Rightarrow z \in \mathbb{N}$ (resp $\mathbb{Z})$. An index family is natural (resp.integral) if all its index sets are natural (resp. integral).

We will use the abbreviation ' $l$ ' for the integral index set $\{(n, 0) ; n \geq l\}$.

The Pullback and Pushforward Theorems, proved in [24] guarantee that polyhomogeneity is preserved under pullbacks and pushforwards by b-fibrations. We now recast these results in the language of $D$-densities and also discuss the interior p-submanifold case. Let $f: X \rightarrow Y$ be a b-fibration between manifolds with corners with degrees and define the 'excess' of any boundary hypersurface (with respect to $f$ ) by $\operatorname{ex}(G)=d(G)-d(H)$ for $G \in M_{1}(X)$ if $f(G)=H \in M_{1}(Y), \operatorname{ex}(G)=d(G)$ if $f(G)=Y$. The defines an integral index family ex on $X$.

Theorem 2.2 (Pullback theorem). Any b-fibration, $f$, induces a pullback map on functions

$$
f^{*}: \mathcal{A}_{\mathrm{phg}}^{\mathcal{J}}(Y) \rightarrow \mathcal{A}_{\mathrm{phg}}^{f^{\#} \mathcal{J}}(X)
$$

where $f^{\#}(\mathcal{J})(G)=0$ if $f(G)=Y$, or $f^{\#}(\mathcal{J})(G)=\{(e(G, H) z+q, k) ;(z, k) \in$ $\mathcal{J}(H), q \in \mathbb{N}\}$ if $f(G)=H \in M_{1}(Y)$.

Theorem 2.3 (Pushforward theorem). If $\operatorname{Re} \mathcal{K}(G)>d(G)$ for all $G$ such that $f(G)=Y$, then the pushforward by a b-fibration $f$, that is, integration over the fibres of $f$, of smooth compactly supported densities extends to a map

$$
f_{*}: \mathcal{A}_{\mathrm{phg}}^{\mathcal{K}}\left(X ; \Omega_{D}(X)\right) \rightarrow \mathcal{A}_{\mathrm{phg}}^{f_{\#}(\mathcal{K}-\text { ex })}\left(Y ; \Omega_{D}(Y)\right)
$$


where, for any index family $\mathcal{E}, f_{\#}(\mathcal{E})(H)=\left\{(z, p) ; \exists G_{1} \ldots G_{k}\right.$ mapping to $H$ and $p_{1} \ldots p_{k}$ such that $\left(z / e\left(G_{i}, H\right), p_{i}\right) \in \mathcal{E}\left(G_{i}\right)$ and $\left.p=p_{1}+\cdots+p_{k}+(k-1)\right\}$.

Note that if $f$ is a simple b-fibration, then the operators $f^{\#}$ and $f_{\#}$ preserve naturality and integrality.

The assumption on the index sets, that $\operatorname{Re} \mathcal{K}(G)>d(G)$ for boundary hypersurfaces mapped onto $Y$, ensures the integrability of $u \in$ $\mathcal{A}_{\text {phg }}^{\mathcal{K}}\left(X ; \Omega_{D}(X)\right)$ over fibres $f^{-1}(p)$ for $p$ in the interior of $Y$, and hence that the pushforward is defined. In fact this condition can be dropped at the expense of considering a Hadamard-regularized pushforward. To define this regularization, we first define a regularized integral, which we call the 'bintegral'. Consider a manifold with corners with hypersurfaces $H_{1}, \ldots, H_{k}$ and boundary defining functions $\rho_{1}, \ldots, \rho_{k}$; then the integral

$$
I\left(\epsilon_{1}, \ldots, \epsilon_{k}\right)=\int_{\rho_{i}>\epsilon_{i}} u, u \in \mathcal{A}_{\mathrm{phg}}^{\mathcal{K}}\left(X ; \Omega_{b}(X)\right)
$$

is polyhomogeneous in $\epsilon_{1}, \ldots, \epsilon_{k}$. The b-integral of $u$, denoted $\int^{b} u$, is by definition the coefficient of the constant term $\epsilon_{1}^{0} \ldots \epsilon_{k}^{0}$ in the asymptotic expansion of $I\left(\epsilon_{1}, \ldots, \epsilon_{k}\right)$. In general it depends on the choice of boundary defining functions but in case $u$ is integrable it reduces to the ordinary integral. The b-pushforward (under any $C^{\infty}$ map) can then be defined by duality:

$$
{ }^{b} f_{*} u(\phi)={ }^{b}\left(u f^{*} \phi\right) \forall \phi \in C^{\infty}(Y) .
$$

The proof of the pushfoward theorem given in [24] extends easily to show that this b-pushforward still satisfies (2.3).

It is useful to have an explicit expression for $\int^{b} u$, when $u$ is not absolutely integrable. We do so only for $u$ a polyhomogeneous distribution on $\mathbb{R}^{+}$since the result extends easily to the general case. It is also sufficient to define the $b$-integral for just a single term of the expansion of $u$ multiplied by a cut-off function $\phi(x)$, where $\phi(x)=1$ near zero and has compact support. The general term to be defined is $\int x^{z}(\log x)^{k} \phi(x) d x / x$. We shall only need the case $k=0$. Consider the integral from $\epsilon$ to infinity, and integrate by parts to get

$$
\left\{\begin{array}{l}
\int_{\epsilon}^{\infty} x^{z} \phi(x) \frac{d x}{x}=-\int_{\epsilon}^{\infty} \frac{x^{z}}{z} x \phi^{\prime}(x) \frac{d x}{x}-\frac{\epsilon^{z}}{z}, z \neq 0 \\
\int_{\epsilon}^{\infty} \phi(x) \frac{d x}{x}=-\int_{\epsilon}^{\infty}(\log x) x \phi^{\prime}(x) \frac{d x}{x}-\log \epsilon
\end{array}\right.
$$


Since $\phi^{\prime}$ is supported away from zero, the integral on the right in either case is convergent for any $z$. The other term on the right is polyhomogeneous in $\epsilon$ with no constant term, so does not contribute to the answer. The b-integral is therefore given by

$$
\left\{\begin{array}{l}
\int_{b}^{b} x^{z} \phi(x) \frac{d x}{x}=-\int_{0}^{\infty} \frac{x^{z}}{z} x \phi^{\prime}(x) \frac{d x}{x}, z \neq 0 \\
\int \phi(x) \frac{d x}{x}=-\int_{0}^{\infty}(\log x) x \phi^{\prime}(x) \frac{d x}{x} .
\end{array}\right.
$$

There are similar expressions when $k \neq 0$.

The two Theorems above give the exponents in the asymptotic expansion of a pullback or pushforward. Next we discuss the coefficients. The coefficient functions in the expansion of the pullback $f^{*} h$ at a boundary hypersurface $G$ in the first theorem are given by the pullbacks of the coefficients for the expansion of $h$ at $H=f(G)$, provided $f^{*}\left(\rho_{H}\right)=\rho_{G}$. For a pushforward, the relationship between the coefficients in the expansions for $u$ and $f_{*} u$ can be rather complicated. We shall compute the coefficients in the expansions of a pushforward $f_{*} u$, at a hypersurface $H$ under the assumption that $u$ is a polyhomogeneous $D$-density on $X$ with $C^{\infty}$ index family and $f: X \rightarrow Y$ is a b-fibration under which either one or two boundary hypersurfaces of $X$ map to $H$.

We start with the 'trivial' case. Thus suppose first that $H \in M_{1}(Y)$ and there is a unique $G \in M_{1}(X)$. with $f(G)=H$. Furthermore, suppose that $e_{f}(G, H)=1$. Let $r$ be a boundary defining function for $H$, so $x=f^{*} r$ is a boundary defining function for $G$. Take a product decomposition of $Y$ near $H$. Then there is a unique product decomposition of $X$ near $G$ in terms of which $f$ becomes a fixed b-fibration, $f_{G}$, from one factor of this decomposition on $X$ to one factor of the decomposition on $Y$. Consider $u \in \mathcal{A}_{\text {phg }}^{\mathcal{K}}\left(X ; \Omega_{b}(X)\right)$ where the index family $\mathcal{K}=l=\{(i, 0) ; i \geq l\}$, at $G$ and suppose initially that $\operatorname{ex}(G)=d(H)=0$. The expansion of $u$ at $G$ takes the form

$$
u \sim \sum_{i \geq l} x^{i} a_{i}\left|\frac{d x}{x}\right|, a_{i} \in \mathcal{A}_{\mathrm{phg}}^{\mathcal{K}(G)}\left(X ; \Omega_{b}(X)\right)
$$

where $\mathcal{K}(G)$ is the induced index family on $G$. By assumption in Theorem 2.3 the elements $a_{i}$ are integrable on the fibres of $f_{G}$. Then the expansion of $u$ at $H$, in terms of the chosen product decomposition is

$$
f_{*} u \sim \sum_{i \geq l} r^{i}\left(f_{G}\right)_{*}\left(a_{i}\right)
$$


In this case the coefficients of $f_{*} u$ are obtained by pushforward of the coefficients of $u$. In the interior of $H$ the pushforward under $f_{G}$ is just an integral over a compact manifold with corners.

Next consider the less trivial case in which $f: X \longrightarrow Y$ is a b-fibration, $H \in M_{1}(Y)$ and

$$
f^{-1} H=G_{1} \cup G_{2}, e_{f}\left(G_{1}, H\right)=e_{f}\left(G_{2}, H\right)=1, G_{1} \cap G_{2}=K \in M_{2}(X) .
$$

Let $r$ be a boundary defining function for $H$, and, for $i=1,2$, let $x_{i}$ be boundary defining functions for $G_{i}$ with $f^{*} r=x_{1} x_{2}$. There are product neighbourhoods in $X$ near $G$, of the form $[0, \delta)_{x_{1}} \times[0, \delta)_{x_{2}} \times K$ and in $Y$ of $H$, of the form $\left[0, \delta^{\prime}\right) \times H$ with $\delta, \delta^{\prime}>0$, such that $f$ is locally the product map

$$
f\left(x_{1}, x_{2}, q\right)=\left(x_{1} x_{2}, f_{K}(q)\right),
$$

where $f_{K}: K \rightarrow H$ is a fibration. Now consider an index family for $X$ with $\mathcal{K}\left(G_{j}\right)$ for $j=1,2$ the integral index sets $\left\{(k, 0) ; k \geq l_{j}\right\}$. As before, without loss of generality, we can assume that $\operatorname{ex}\left(G_{i}\right)=d\left(G_{i}\right)=0$. If $u \in$ $\mathcal{A}_{\text {phg }}^{\mathcal{K}}\left(X ; \Omega_{b}(X)\right)$ then the expansions for $u$ at $G_{1}$ and $G_{2}$ in terms of this product decomposition may be written

$$
\begin{aligned}
u & \sim \sum_{j \geq l_{1}}\left(x_{1} x_{2}\right)^{j} a_{j}\left|\frac{d x_{1}}{x_{1}}\right| \text { at } G_{1}, \text { where } a_{j} \sim \sum_{k \geq l_{2}} x_{2}^{k-j} c_{j k}\left|\frac{d x_{2}}{x_{2}}\right| \text { at } G_{2} ; \\
u & \sim \sum_{k \geq l_{2}}\left(x_{1} x_{2}\right)^{k} b_{k}\left|\frac{d x_{2}}{x_{2}}\right| \text { at } G_{2} \text {, where } b_{k} \sim \sum_{j \geq l_{1}} x_{1}^{j-k} c_{j k}\left|\frac{d x_{1}}{x_{1}}\right| \text { at } G_{1} .
\end{aligned}
$$

Here the $c_{j k} \in \mathcal{A}_{\text {phg }}^{\mathcal{K}_{K}}\left(K ; \Omega_{b}(K)\right)$ with $\mathcal{K}_{K}$ the induced index family for $K$.

Lemma 2.4. Under the assumptions of the preceding paragraph, if $u \in \mathcal{A}_{\mathrm{phg}}^{\mathcal{K}}\left(X ; \Omega_{b}(X)\right)$ where $\mathcal{K}$ satisfies the integrability conditions of Theorem 2.3 and has support in the product neighbourhood of $K=G_{1} \cap G_{2}$, then the expansion of $f_{*} u$ at $H$ is

$$
\begin{aligned}
f_{*} u \sim \sum_{j \geq l_{1}} r^{j b}\left(f_{G_{1}}\right)_{*} a_{j}\left|\frac{d r}{r}\right| & \\
& +\sum_{k \geq l_{2}} r^{k b}\left(f_{G_{2}}\right)_{*} b_{k}\left|\frac{d r}{r}\right|-\sum_{j \geq \max l_{1}, l_{2}} r^{j} \log r^{b}\left(f_{K}\right)_{*} c_{j j}\left|\frac{d r}{r}\right|
\end{aligned}
$$


where $f_{G_{1}}, f_{G_{2}}$ and $f_{K}$ are, respectively, the b-fibrations with range $H$ obtained by restricting $f$ to $G_{1}, G_{2}$ and $K$.

Proof. Theorem 2.3 shows that there is an expansion of the form (2.11). It suffices therefore to compute the coefficients up to a given order, i.e. the coefficients of $r^{p} \log r$ and $r^{p}$ for $p<N$. Multiplying $u$ by $r^{-N}=\left(x_{1} x_{2}\right)^{-N}$ we may assume $N=0$, at the expense of changing $l_{1}$ and $l_{2}$. Since $l_{1}$ and $l_{2}$ are arbitrary it is enough to compute the coefficients of $r^{p}$ and $r^{p} \log r$ for $p<0$.

Suppose first that $l_{1}$ is large, say $l_{1}>\left|l_{2}\right|$. Then all terms are integrable at $G_{1}$. It follows by continuity from (2.7) that $f_{*} u$ is given by $\sum_{k=l_{2}}^{0}\left(f_{G_{2}}\right)_{*} b_{k}+$ $o(1)$, which verifies (2.11) in this case. Thus it is enough to consider an individual term in the expansion at $G_{1}$ in (2.10)

$$
\begin{aligned}
& u=\left(x_{1} x_{2}\right)^{j} a_{j} \phi\left(x_{1}\right)\left|\frac{d x_{1}}{x_{1}}\right| \\
& \text { where } \mathcal{A}_{\mathrm{phg}}^{\mathcal{K}\left(G_{1}\right)}\left(G_{1} ; \Omega_{b}\right) \ni a_{j} \sim \sum_{k \geq l_{2}} x_{2}^{k-j} c_{j k}\left|\frac{d x_{2}}{x_{2}}\right| \text { at } G_{2}
\end{aligned}
$$

and $\phi\left(x_{1}\right)$ has compact support and is identically equal to 1 near $x_{1}=0$. The same continuity argument applies to the terms in the expansion for $a_{j}$ in (2.12) beyond the point of integrability at $G_{2}$. Thus in fact it is enough to consider a single term of the form

$$
u=x_{1}^{j} x_{2}^{k} c_{j k} \phi\left(x_{1}\right) \phi\left(x_{2}\right)\left|\frac{d x_{1} d x_{2}}{x_{1} x_{2}}\right|
$$

$$
\text { where } c_{j k} \in \mathcal{A}_{\mathrm{phg}}^{\mathcal{K}_{K}}\left(G_{1}\right)
$$

and by assumption the induced index family $\mathcal{K}_{K}$ is such that $c_{j k}$ is integrable on $K=G_{1} \cap G_{2}$.

By symmetry it can be assumed that $j \geq k$ in (2.13). Then the integral for $r>0$ is

$$
r^{k} \int \phi\left(x_{1}\right) \phi\left(\frac{r}{x_{1}}\right) x_{1}^{j-k} \frac{d x_{1}}{x_{1}} \cdot\left({ }^{b}\left(f_{K}\right)_{*} c_{j k}\right) .
$$

If $j>k$ then integration by parts changes the $x_{1}$ integral in (2.14) to

$$
-r^{k} \int x_{1} \phi^{\prime}\left(x_{1}\right) \phi\left(\frac{r}{x_{1}}\right) \frac{x_{1}^{j-k}}{j-k} \frac{d x_{1}}{x_{1}}-r^{k} \int \phi\left(x_{1}\right) x_{1} \partial_{x_{1}} \phi\left(\frac{r}{x_{1}}\right) \frac{x_{1}^{j-k}}{j-k} \frac{d x_{1}}{x_{1}} .
$$


For $r$ small, $\phi\left(r / x_{1}\right)=1$ on the support of $x_{1} \phi^{\prime}\left(x_{1}\right)$ and similarly $\phi\left(x_{1}\right)=1$ on the support of $\partial_{x_{1}} \phi\left(r / x_{1}\right)$. Changing the variable of integration in the second integral to $x_{2}$ gives

$$
-r^{k} \int x_{1} \phi^{\prime}\left(x_{1}\right) \frac{x_{1}^{j-k}}{j-k} \frac{d x_{1}}{x_{1}}-r^{j} \int x_{2} \phi^{\prime}\left(x_{2}\right) \frac{x_{2}^{k-j}}{k-j} \frac{d x_{2}}{x_{2}} .
$$

If $j=k$ then the same integration by parts can be used, starting from $1=x_{1} \partial / \partial x_{1} \log x_{1}$, can be used to reduce (2.14) to

$$
\begin{gathered}
-r^{k} \int x_{1} \phi^{\prime}\left(x_{1}\right) \phi\left(\frac{r}{x_{1}}\right) \log x_{1} \frac{d x_{1}}{x_{1}}-r^{k} \int \phi\left(x_{1}\right) x_{1}\left(\partial_{x_{1}} \phi\left(\frac{r}{x_{1}}\right)\right) \log x_{1} \frac{d x_{1}}{x_{1}} \\
=r^{k}\left(-\log r-2 \int_{0}^{\infty} x_{1} \phi^{\prime}\left(x_{1}\right) \log x_{1} \frac{d x_{1}}{x_{1}}\right) .
\end{gathered}
$$

In either case, comparing with (2.6), we get the appropriate coefficients in (2.11). This completes the proof of the lemma.

Finally let us write out the result of Lemma 2.4 when $d(H)=d$ and $\operatorname{ex}\left(G_{1}\right)=e_{1}$ and $\operatorname{ex}\left(G_{2}\right)=e_{2}$ are arbitrary integers. In this case, $u$ has an expansion of the form

$$
\begin{aligned}
& u \sim \sum_{j \geq l_{1}}\left(x_{1} x_{2}\right)^{j} a_{j}\left|\frac{d x_{1}}{x_{1}\left(x_{1} x_{2}\right)^{d+e_{1}}}\right| \text { at } G_{1}, \\
& \text { where } a_{j} \sim \sum_{k \geq l_{2}} x_{2}^{k-j} c_{j k}\left|\frac{d x_{2}}{x_{2}^{1+e_{2}-e_{1}}}\right| \text { at } G_{2} ; \\
& u \sim \sum_{k \geq l_{2}}\left(x_{1} x_{2}\right)^{k} b_{k}\left|\frac{d x_{2}}{x_{2}\left(x_{1} x_{2}\right)^{d+e^{2}}}\right| \text { at } G_{2} \\
& \text { where } b_{k} \sim \sum_{j \geq l_{1}} x_{1}^{j-k} c_{j k}\left|\frac{d x_{1}}{x_{1}^{1+e_{1}-e_{2}}}\right| \quad \text { at } G_{1} .
\end{aligned}
$$

and then the result of pushing forward by $f$ is

$$
\begin{array}{r}
f_{*} u \sim \sum_{j \geq l_{1}-e_{1}} r^{j b}\left(f_{G_{1}}\right)_{*} a_{j+e_{1}}\left|\frac{d r}{r^{1+d}}\right|+\sum_{k \geq l_{2}-e_{2}} r^{k b}\left(f_{G_{2}}\right)_{*} b_{k+e_{2}}\left|\frac{d r}{r^{1+d}}\right| \\
-\sum_{j \geq \max l_{1}-e_{1}, l_{2}-e_{2}} r^{j} \log r^{b}\left(f_{K}\right)_{*} c_{j+e_{1}, j+e_{2}}\left|\frac{d r}{r}\right|
\end{array}
$$


Although the b-integral depends, mildly, on the choice of boundary defining functions used in its definition we will work below with explicit boundary defining functions so this dependence will not be indicated in the notation. If the b-integral formally computes the trace of a smoothing operator $A$, then the regularized integral is denoted $\mathrm{b}-\operatorname{Tr}(A)$. The b-eta invariant is defined in terms of the b-Trace of the operator $\partial e^{-t \widetilde{\varpi}^{2}}$ (see (9.2)). For this operator, we will show in section 9 that the Schwartz kernel of the pointwise trace of this operator vanishes on the boundary, so the integral actually converges. Hence, the b-eta invariant is well defined, independent of any choice of boundary defining function.

The pushforward theorem from [24], discussed above, can be extended to densities on $X$ which have interior conormal singularities along a psubmanifold $S$ transverse to all boundary hypersurfaces of $X$ and to $f$. We denote the space of such densities with conormal order $m$ at $S$ by $I^{m} \mathcal{A}_{\text {phg }}^{\mathcal{K}}\left(X ; \Omega_{D}(X) ; S\right)$. To see why this modification is permissible, first note that by a standard result about wavefront sets (see [16] for example) $f_{*} u$ is smooth in the interior of $Y$. To understand the situation at the boundary, consider the proof of the pushforward theorem in [24]. The idea there is to remove terms in the asymptotic expansion of $u$ at each boundary hypersurface $G$ of $X$ up to any given finite order using test differential operators $\mathcal{B}(\mathcal{K}, \mathfrak{s})$ which are appropriate combinations of compositions of any given nonvanishing b-vector field $r_{G}$ normal to $G$. Since both $f$ and any $G \in M_{1}(X)$ are transverse to $S$, if $G$ intersects $S$ nontrivially and $f(G)=H$, any normal vector field $r_{H}$ is $f$-related to a normal vector field $r_{G}$ on $X$ tangent to $S$. Using this $r_{G}$ in $\mathcal{B}(\mathcal{K}, \mathfrak{s})$ shows that this test differential operator removes the leading terms in the asymptotic expansion of $u$ at $G$ while preserving the order of conormality at $S$. This shows the image space is the same as in Theorem 2.3.

\subsection{Two Blowup Lemmas.}

The important properties of b-fibrations have been discussed in section 1.4. In modifying the spaces of Part I we often face the situation where we have a b-fibration $f: X \rightarrow Y$ and we perform blowups on $X$ or $Y$, obtaining new spaces $\hat{X}, \hat{Y}$. We would like $f$ to lift to a b-fibration $\hat{f}: \hat{X} \rightarrow \hat{Y}$. The first and second lemmas below give conditions under which one can regain a b-fibration after such a blowup in $X$ and $Y$ respectively.

Lemma 2.5. Let $f: X \longrightarrow Y$ be a $b$-fibration between compact manifolds with corners and suppose that $T \subset Y$ is a closed p-submanifold such that for 
each boundary hypersurface $H \subset Y$ intersecting $T$, and each $G \in M_{1}(X)$, either $e_{f}(G, H)=0$ or $e_{f}(G, H)=1$. Then, with $\mathcal{S}$ the minimal collection of $p$-submanifolds of $X$ into which the lift of $T$ under $f$ decomposes, $f$ extends from the complement of $f^{-1}(T)$ to a $b$-fibration

$$
f_{T}:[X, \mathcal{S}] \longrightarrow[Y, T]
$$

for any order of blow up of the elements of $\mathcal{S}$.

Proof. The result is local in nature, so we may restrict attention to neighbourhoods of $q \in T$ and $p \in X$ such that $f(p)=q$. Choose coordinates $x_{1}^{\prime}, \ldots, x_{k^{\prime}}^{\prime}, y_{1}^{\prime}, \ldots, y_{n^{\prime}-k^{\prime}}^{\prime}$ near $q$, such that $q=(0, \ldots, 0)$, the $x_{i}^{\prime}$ are nonnegative, the $y_{j}^{\prime}$ take values in $(-\epsilon, \epsilon)$ and in terms of which $T=\left\{x_{1}^{\prime}=\right.$ $\left.\cdots=x_{l}^{\prime}=y_{1}^{\prime}=\cdots=y_{m}^{\prime}=0\right\}$. Because of the assumption on the boundary exponents of $f$, it is possible to choose coordinates $x_{1}, \ldots, x_{k}, y_{1}, \ldots, y_{n-k}$ near $p \in X$ so that

$$
f^{*} x_{i}^{\prime}=\prod_{r \in I_{i}} x_{r}, 1 \leq i \leq l \text { and } f^{*} y_{j}^{\prime}=y_{j}, 1 \leq j \leq n^{\prime}-k^{\prime}
$$

with the $I_{i} \subset\{1 \ldots k\}$ nonempty and disjoint.

Since $f$ is a b-fibration, necessarily $k^{\prime} \geq k$ and $n^{\prime}-k^{\prime} \leq n-k$.

In these coordinates

$$
f^{-1}(T)=\left\{\prod_{r \in I_{i}} x_{r}=0,1 \leq i \leq l \text { and } y_{j}=0,1 \leq j \leq m\right\} .
$$

Thus an element of $\mathcal{S}$, the collection of p-submanifolds into which $f^{-1}(T)$ decomposes, is determined by the choice of a single index from each of the $I_{i}$. Choice of an ordering $S_{1}, \ldots, S_{N}$ of the elements of $\mathcal{S}$ gives

$$
S_{k}=\left\{x_{k_{1}}=\cdots=x_{k_{l}}=y_{1}=\cdots=y_{m}=0\right\}
$$

with $k_{i} \in I_{i}$. Thus $N$ is the product over $i$ of the number of elements in $I_{i}$ and for each $k$ with $1 \leq k \leq N, k_{i}$ is the unique element of $I_{i}$ such that $x_{k_{i}}$ vanishes on $S_{k}$.

Consider the action of blowing up $S_{1}$. This replaces $S_{1}$ by its inward pointing spherical normal bundle. The function $R_{1}=x_{1_{1}}+\cdots+x_{1_{l}}+\left(y_{1}^{2}+\right.$ 
$\left.\cdots+y_{m}^{2}\right)^{1 / 2}$ defines the new boundary hypersurface so introduced. Now consider the functions

$$
\begin{aligned}
& x_{i}^{(1)}=\left\{\begin{array}{l}
\frac{x_{i}}{R_{1}} \text { if } i=1_{j} \text { for some } j \\
x_{i} \text { otherwise }
\end{array}\right. \\
& y_{j}^{(1)}=\left\{\begin{array}{l}
\frac{y_{j}}{R_{1}} \text { if } 1 \leq i \leq m \\
y_{j} \text { otherwise. }
\end{array}\right.
\end{aligned}
$$

Observe that $x_{1_{1}}^{(1)}+\cdots+x_{1_{l}}^{(1)}+\left(\left(y_{1}^{(1)}\right)^{2}+\left(y_{m}^{(1)}\right)^{2}\right)^{1 / 2}=1$ and that $d x_{1_{i}}^{(1)} \neq 0$ unless $x_{1_{i}}^{(1)}=1$ and similarly, $d y_{j}^{(1)} \neq 0$ unless $y_{j}^{(1)}= \pm 1$ if $1 \leq j \leq m$. Away from the front face of $\left[X ; S_{1}\right]$ nothing has changed and near each point of this new boundary hypersurface $R_{1}$ and some $n-1$ of the $n$ functions $x_{i}^{(1)}$, $y_{j}^{(1)}$ form a coordinate system, the one function excluded being non-zero. The lifts to $\left[X, S_{1}\right]$ of the submanifolds $S_{k}, k \geq 2$ are therefore given by the vanishing of the functions $x_{k_{1}}^{(1)}, \ldots, x_{k_{l}}^{(1)}, y_{1}^{(1)}, \ldots, y_{m}^{(1)}$, which, since they vanish there, must be amongst the coordinates at each point of the lifted submanifold.

Thus, after the first blowup the combinatorial arrangement is as before, with one less submanifold $S_{k}$. We can therefore proceed to blow up $S_{2}$, $S_{3}, \ldots, S_{N}$ and define successive functions

$$
\begin{aligned}
R_{k} & =x_{k_{1}}^{(k-1)}+\cdots+x_{k_{l}}^{(k-1)}+\left(\left(y_{1}^{(k-1)}\right)^{2}+\cdots+\left(y_{m}^{(k-1)}\right)^{2}\right)^{\frac{1}{2}} \\
x_{i}^{(k)} & =\left\{\begin{array}{l}
\frac{x_{i}^{(k-1)}}{R_{k}} \text { if } i=k_{j} \text { for some } j \\
x_{i}^{(k-1)} \text { otherwise }
\end{array}\right. \\
y_{j}^{(k)} & =\left\{\begin{array}{l}
\frac{y_{j}^{(k-1)}}{R_{k}} \text { if } 1 \leq i \leq m \\
y_{j}^{(k-1)} \text { otherwise. }
\end{array}\right.
\end{aligned}
$$

Then the $R_{k}$ for $k=1, \ldots, N$ are defining functions for the blown up surfaces.

Consider the map

$$
f_{T}^{\prime}:[X, \mathcal{S}] \longrightarrow Y, f_{T}^{\prime}=f \circ \beta_{X}
$$

where $\beta_{X}:[X, \mathcal{S}] \longrightarrow X$ is the total blowdown map. The coordinates pull back to be of the form

$$
\begin{gathered}
\left(f_{T}^{\prime}\right)^{*} x_{i}^{\prime}=\prod_{r \in I_{i}} \beta_{X}^{*} x_{r}=\prod_{r \in I_{i}}\left[x_{r}^{(N)} \prod_{k \text { s.t. } k_{i}=r} R_{k}\right]=R_{1} \ldots R_{N} \prod_{r \in I_{i}} x_{r}^{(N)} \\
\left(f_{T}^{\prime}\right)^{*} y_{j}^{\prime}=R_{1} R_{2} \cdots R_{N} y_{j}^{(N)} .
\end{gathered}
$$


Thus, $R^{\prime}=x_{1}^{\prime}+\cdots+x_{l}^{\prime}+\left({y^{\prime}}_{1}^{2}+\cdots+y_{m}^{\prime 2}\right)^{1 / 2}$ lifts to

$$
R_{1} \cdots R_{N}\left(\sum_{i=1}^{l}\left(\prod_{r \in I_{i}} x_{r}^{(N)}\right)+\left(\sum_{j=1}^{m}\left(y_{j}^{(N)}\right)^{2}\right)^{1 / 2}\right) .
$$

The right factor does not vanish. Indeed, it would vanish only at a point where some $x_{r_{i}}^{(N)}, r_{i} \in I_{i}$ for each $i$, and each $y_{j}^{(N)}$ vanished. But the choice $\left\{r_{i}\right\}$ corresponds to some submanifold $S_{k}$ and after $S_{k}$ is blown up, $\sum x_{r_{i}}^{(k)}+$ $\left(\sum\left(y_{j}^{(k)}\right)^{2}\right)^{1 / 2}=1$. Thus

$$
\begin{aligned}
& \left(f_{T}^{\prime}\right)^{*} R^{\prime}=a \cdot R_{1} \ldots R_{N}, \\
& \left(f_{T}^{\prime}\right)^{*} \frac{x_{i}^{\prime}}{R^{\prime}}=a_{i} \cdot \prod_{r \in I_{i}} x_{r}^{(N)} \text { and } \\
& \left(f_{T}^{\prime}\right)^{*} \frac{y_{j}^{\prime}}{R^{\prime}}=a_{j}^{\prime} \cdot y_{j}^{(N)}
\end{aligned}
$$

where $a, a_{i}$ and $a_{j}^{\prime}$ are smooth positive functions. This shows that the map (2.18) lifts to a map (2.16) which is a b-fibration.

Following the proof of Lemma 2.5 we have also shown:

Corollary 2.6. Under the conditions of Lemma 2.5, if $f$ is simple then so is $f_{T}$ in (2.16).

For the next lemma, consider the relative b-tangent space of a p-submanifold. For a p-submanifold $S$, of a manifold with corners, $X$, the (relative) b-tangent space ${ }^{b} T_{p}(S, X) \subset{ }^{b} T_{p} X$ at $p \in S$ is the linear space of values at $p$ of those elements of $\mathcal{V}_{b}(X)$ which are tangent to $S$. Its dimension is $\operatorname{dim} S+k$ where $k$ is the codimension of the smallest boundary face, $\mathrm{Fa}(S)$, containing $S$ (so $k=0$ if $S$ is an interior p-submanifold). These spaces form a bundle ${ }^{b} T(S, X)$ over $S$ and the quotient by ${ }^{b} \mathrm{NFa}(S)$, the b-normal space to $\mathrm{Fa}(S)$, is canonically isomorphic to the (intrinsic) b-tangent bundle to $S$ :

$$
{ }^{b} T(S, X) /{ }^{b} N_{S} \mathrm{Fa}(S) \cong{ }^{b} T S .
$$

Lemma 2.7. Let $f: X \longrightarrow Y$ be a b-fibration of compact manifolds with corners and suppose that $S \subset X$ is a closed p-submanifold to which $f$ is $b$-transversal, in the sense that

$$
\operatorname{Ker}\left(\left.{ }^{b} f_{*}\right|_{b} T_{p} X\right)+{ }^{b} T_{p}(S, X)={ }^{b} T_{p} X \forall p \in S,
$$


and such that $f(S)$ is not contained in any boundary face of $Y$ of codimension 2. Then the composition of the blowdown map $\beta:[X, S] \longrightarrow X$ with $f$ is a $b$-fibration

$$
f^{\prime}:[X, S] \longrightarrow Y
$$

Proof. The b-tangent map of the blowdown map $\beta$ maps onto ${ }^{b} T_{\beta(p)}(S, X)$ for each point $p \in \beta^{-1} S$. The b-transversality condition implies that composition with ${ }^{b} f_{*}$ maps onto ${ }^{b} T_{f(\beta(p))} X$, so $f^{\prime}$ is a b-submersion. The condition on $f(S)$ means that $f(S)$ is a boundary face of codimension 1 or 0 , so that $f^{\prime}$ is actually a b-fibration.

In view of (2.19) the condition of b-transversality in (2.20) is equivalent to the b-transversality of $\left.f\right|_{\mathrm{Fa}}(S)$, as a b-fibration onto $f(\mathrm{Fa}(S))$, to $S$ as a submanifold of $\mathrm{Fa}(S)$. This can also be restated as the condition that $f$ restricts to $S$ to a b-fibration onto $f(S)$, which is often simple to check.

\subsection{Logarithmic blow up.}

To handle the logarithmic behaviour of the surgery problem, when the boundary operator is not invertible, we introduce the notion of logarithmic blow up. If $X$ is a compact manifold with corners and $\rho_{H} \in C^{\infty}(X)$ defines $H \in M_{1}(X)$, then consider

$$
\begin{array}{r}
C^{\infty}\left([X, H]_{\log }\right)=\left\{g\left(\operatorname{ilg} \rho_{H}, f_{1}, \ldots, f_{p}\right) ; g \in C^{\infty}\left(\mathbb{R}^{p+1}\right), f_{i} \in C^{\infty}(X)\right\} \\
\text { where } \operatorname{ilg} \rho_{H}=\frac{1}{\log \left(1 / \rho_{H}\right)} .
\end{array}
$$

This is a new $C^{\infty}$ structure on $X$, albeit diffeomorphic to the original one. In fact this $C^{\infty}$ structure is independent of the choice of defining function $\rho_{H}$, and so defines $[X, H]_{\log }$. Since $\rho_{H}$ is a $C^{\infty}$ function of ilg $\rho_{H}$, namely

$$
\rho_{H}=\exp \left(-\frac{1}{\operatorname{ilg} \rho_{H}}\right),
$$

the identity map on $X$ is smooth as a map $\beta_{\log }:[X, H]_{\log } \longrightarrow X$. Clearly the operations of logarithmic blow up of two or more hypersurfaces commute. This allows us to define unambiguously the 'total logarithmic blow-up' $X_{\log }$ of $X$ by blowing up each of the boundary hypersurfaces. 
Perhaps surprisingly, an appropriate combination of the non-algebraic notion of logarithmic blowup with certain (ordinary) blow ups behaves well with respect to certain b-fibrations. To illustrate this we give a simple example.

Example 1. Consider the b-fibration $g: X \longrightarrow Y$ where $X=[0, \infty)^{2}, Y=$ $[0, \infty)$ and $g\left(x_{1}, x_{2}\right)=x_{1} x_{2}$. In terms of the boundary defining functions $r=\operatorname{ilg} x, \rho_{1}=\operatorname{ilg} x_{1}$ and $\rho_{2}=\operatorname{ilg} x_{2}$, we have, in the interior of $X$

$$
g^{*} r=g^{*} \frac{1}{\log \frac{1}{x}}=\frac{1}{\log \frac{1}{x_{1} x_{2}}}=\frac{1}{\log \frac{1}{x_{1}}+\log \frac{1}{x_{2}}}=\frac{\rho_{1} \rho_{2}}{\rho_{1}+\rho_{2}} .
$$

Thus $g$ does not lift to a smooth map from $X_{\log }$ to $Y_{\log }$. If we further blow up $X$ by defining $\hat{X}=\left[X_{\text {log }} ;(0,0)\right]$ then boundary defining functions for $\hat{X}$ are $\hat{\rho}_{1}=\frac{\rho_{1}}{\rho_{1}+\rho_{2}}, \hat{\rho}_{2}=\frac{\rho_{2}}{\rho_{1}+\rho_{2}}$, and $\hat{\rho}_{3}=\rho_{1}+\rho_{2}$ for the new face. Thus $g^{*} r=\hat{\rho}_{1} \hat{\rho}_{2} \hat{\rho}_{3}$, so it follows that $g$ lifts to a b-fibration $\hat{g}: \hat{X} \longrightarrow Y_{\log }$.

We generalize this result in the next lemma. Before it is stated we need to introduce the notion of 'total boundary blow up'.

\subsection{Total boundary blow up.}

The total boundary blow up, $X_{\mathrm{tb}}$, of a compact manifold with corners, $X$, is defined by blowing up (in the radial sense) all the boundary faces, in order of increasing dimension. Blowing up all faces of dimension $\leq k$ separates the lifts of the faces of dimension $k$, so there are no ambiguities of order in this definition. The boundary hypersurfaces of $X_{\mathrm{tb}}$ are parametrized by $M^{\prime}(X)$, the set of proper boundary faces of $X$. In the next lemma we consider the effect of the combined operations of logarithmic blowup and total boundary blowup on simple b-fibrations. Since this combination occurs quite frequently in the sequel we introduce the following notation for the 'logarithmic total boundary blowup' of a compact manifold with corners $X$

$$
X_{1 \mathrm{t}}=\left(X_{\log }\right)_{\mathrm{tb}}
$$

Lemma 2.8. Let $f: X \longrightarrow Y$ be a simple b-fibration of compact manifolds with corners. Then $f$ lifts from the interior to a simple b-fibration $f_{1 \mathrm{lt}}$ : $X_{\mathrm{lt}} \longrightarrow Y_{\mathrm{lt}}$.

This result is applied in subsequent subsections to the surgery spaces of Part I to obtain new 'logarithmic surgery spaces'. 
Proof. Again this is a local result. Using Section 2 of [24], we can assume that $f$ takes the following form in local coordinates

$$
\left(x_{1}, \ldots x_{k}, y_{1}, \ldots y_{n-k}\right) \longrightarrow\left(\prod_{i \in I_{1}} x_{i}, \ldots, \prod_{i \in I_{k}} x_{i}, y_{1}, \ldots y_{n^{\prime}-k^{\prime}}\right)
$$

The condition (2.2) implies that the $I_{i}$ form a partition of $\{1, \ldots, k\}$. If $g: X^{\prime} \longrightarrow Y^{\prime}$ is a fibration of manifolds without boundary then the result holds for $f \times g$ if it holds for $f$. Thus the factors of $\mathbb{R}^{n-k}$ in the domain and $\mathbb{R}^{n^{\prime}-k^{\prime}}$ in the range can be dropped and it suffices to prove the result for maps of the form

$$
\left(x_{1}, \ldots x_{k}\right) \rightarrow\left(\prod_{i_{1} \in I_{1}} x_{i_{1}}, \ldots, \prod_{i_{k} \in I_{k}} x_{i_{k}}\right) .
$$

The composite of two simple b-fibrations is again a simple b-fibration and, the same operations being applied in domain and range, the result holds for the composite if it holds for the factors. The map (2.25) decomposes into the composite of simple b-fibrations of the form

$$
\begin{array}{r}
f: \mathbb{R}_{+}^{k} \longrightarrow \mathbb{R}_{+}^{k-1} \\
\left(x_{1}, \ldots x_{k}\right) \longmapsto\left(x_{1}, \ldots, x_{k-2}, x_{k-1} x_{k}\right)
\end{array}
$$

with appropriate permutation of the coordinates, so we only need to prove the lemma for maps, $f$, of the form (2.26).

With $X=\mathbb{R}_{+}^{k}$ and $Y=\mathbb{R}_{+}^{k-1}$ let $f$ be as in (2.26). Using the Lemma 2.4, or the example preceeding that result, we see that $f$ lifts to a b-fibration $\tilde{f}:\left[X_{\log } ; K\right] \longrightarrow Y_{\log }$ where $K$ is the lift to the logarithmic space of $\left\{x_{k-1}=\right.$ $\left.x_{k}=0\right\}$. Denote by $\tilde{K}$ the new boundary hypersurface produced by the blowup of $K$. To lift to $X_{\text {lt }}$, we use Lemma 2.5. Thus $Y_{\text {lt }}$ is produced from $Y_{\log }$ by blowing up all codimension $l$ hypersurfaces for $l=(k-1), \ldots, 1$ successively. Denote the lift to $X_{\mathrm{lt}}$ of the hypersurface $x_{i}=0$ by $H_{i}$, and denote by $\mathcal{H}_{l}$ the sequence of blowups $\mathcal{H}_{l, 1}$ followed by $\mathcal{H}_{l, 2}$ followed by $\mathcal{H}_{l, 3}$ where

$$
\begin{aligned}
\mathcal{H}_{l, 1}= & \text { all } l \text {-fold intersections of } H_{1} \ldots H_{k-2} \\
\mathcal{H}_{l, 2}= & \text { all } l \text {-fold intersections of } H_{1} \ldots H_{k-2} \text { and } \tilde{K} \text { involving } \tilde{K} \\
\mathcal{H}_{l, 3}= & \text { all } l \text {-fold intersections of } H_{1} \ldots H_{k} \text { involving } \\
& \text { exactly one of }\left\{H_{k-1}, H_{k}\right\} .
\end{aligned}
$$

Then by Lemma $2.5, \tilde{f}$ lifts to a map

$$
\hat{X}=\left[X_{\log } ; K ; \mathcal{H}_{k-1}, \ldots, \mathcal{H}_{2}\right] \longrightarrow Y_{\mathrm{lt}} .
$$


To show that $\hat{X}=X_{1 t}$ we proceed inductively to show that

$$
\begin{aligned}
\hat{X}= & {\left[X_{\text {log }} ; \text { all faces of codimension } \geq l+1 ;\right.} \\
& \left.\mathcal{H}_{l, 1} ; \mathcal{H}_{l, 3} ; K ; \mathcal{H}_{l-1} \ldots \mathcal{H}_{2}\right] .
\end{aligned}
$$

For $l=k$ this space is $\hat{X}$, for $l=2$ it is $X_{\mathrm{lt}}$. So we assume that the statement is true for some $l, 2<l \leq k$. To show that it is true for $l-1$ we will use Lemma 2.1 and the following separation result:

Lemma 2.9. If all boundary faces $H_{I}, I \subset\{1, \ldots, n\}$, of $X=\mathbb{R}_{+}^{k}$ of codimension $\geq m$ have been blown up (in increasing order of dimension) then the lifts of $H_{\sigma(1)} \cap \cdots \cap H_{\sigma(p+r)}$ and $H_{\sigma(p)} \cap \cdots \cap H_{\sigma(p+s)}$ where $\sigma$ is a permutation and $s \geq r$ are disjoint provided $p+s \geq m$.

Proof. The indicated surfaces are separated when $H_{\sigma(1)} \cap \cdots \cap H_{\sigma(p+s)}$ is blown up.

We now commute the $K$ blowup past the $\mathcal{H}_{l-1}=\left\{\mathcal{H}_{l-1,1}, \mathcal{H}_{l-1,2}, \mathcal{H}_{l-1,3}\right\}$ blowups. Applying this lemma to $\left[X_{\log } ; \mathcal{G}_{l+1}\right]$ where $\mathcal{G}_{l+1}$ is the set of all faces of codimension at least $l+1, K$ is disjoint from all faces in $\mathcal{H}_{l-1,1}$ so the $K$ blowup commutes with the $\mathcal{H}_{l-1,1}$ blowups. Again by Lemma 2.9, any two faces in $\mathcal{H}_{l-1,2}$ are disjoint, and they are all contained in $K$, so by Lemma 2.1 we may do the $\mathcal{H}_{l-1,2}$ blowups first. They are then, by Lemma 2.9 , disjoint from the $\mathcal{H}_{l-1,1}$ faces, so can be commuted past these too. When we do this, they yield with the $\mathcal{H}_{l, 1}$ and $\mathcal{H}_{l, 3}$ blowups all the codimension $l$ faces, so we get

$\hat{X}=\left[X_{\log } ;\right.$ all faces of codimension $\left.\geq l ; \mathcal{H}_{l-1,1} ; K ; \mathcal{H}_{l-1,3} ; \mathcal{H}_{l-2} \ldots \mathcal{H}_{2}\right]$.

Again by applying Lemma $2.9, K$ is disjoint from all $\mathcal{H}_{l-1,3}$ faces so we obtain (2.27) for $l-1$. This completes the induction, so we have shown that $\tilde{f}$ lifts to $f_{\mathrm{lt}}: X_{\mathrm{lt}} \longrightarrow Y_{\mathrm{lt}}$. Finally, both the result of the example and Lemma 2.5 preserve (2.2) so $f_{1 \mathrm{t}}$ is simple.

Let us define degrees (see section 2.1) for spaces of the form $Z_{\mathrm{lt}}$ for a manifold with corners $Z$. We fix a degree function by letting the degree $d(H)$ of a hypersurface $H$ of $Z_{\mathrm{lt}}$ be the codimension of the face of $Z$ of which it is the blowup. We also define the cusp density bundle $\Omega_{c}(X)$ for any manifold with corners $X$ to be $\prod_{H \in M_{1}(X)} \rho_{H}^{-1} \Omega_{b}(X)$; in other words, the degree density bundle where all degrees are equal to 1 . Then the following results hold: 
Lemma 2.10. For any manifold with corners $X$

$$
\begin{aligned}
\beta_{\log }^{*} \Omega_{b}(X) & =\Omega_{c}\left(X_{\log }\right) \\
\beta_{\mathrm{tb}}^{*} \Omega_{c}\left(X_{\log }\right) & =\Omega_{D}\left(X_{\mathrm{lt}}\right) .
\end{aligned}
$$

Proof. Since $d \rho / \rho=d(\operatorname{ilg} \rho) /(\operatorname{ilg} \rho)^{2},(2.28)$ follows. To prove (2.29), first observe that for any $H \in M_{1}\left(X_{\log }\right)$, with boundary defining function $\rho_{H}$,

$$
\beta_{\mathrm{tb}}^{*} \rho_{H}=\prod_{F \subset H} r_{F},
$$

where, if $F$ is a face of codimension $k$ in $X_{\log }$ then $r_{F}$ denotes the boundary defining function for the boundary hypersurface of $X_{1 \mathrm{t}}$ corresponding to $F$.

Under blowup of a boundary face, the b-density bundle lifts to the bdensity bundle. Hence,

$$
\begin{gathered}
\beta_{\mathrm{tb}}^{*} \prod_{H \in M_{1}(X)} \rho_{H}^{-1} \Omega_{b}\left(X_{\mathrm{log}}\right)=\prod_{H \in M_{1}(X)} \prod_{F \subset H} r_{F}^{-1} \Omega_{b}\left(X_{\mathrm{lt}}\right) \\
=\prod_{F \in M^{\prime}(X)} r_{F}^{-\operatorname{codim} F} \Omega_{b}\left(X_{\mathrm{lt}}\right) .
\end{gathered}
$$

If $S \subset M$ is a p-submanifold, let $\left[Z_{1 \mathrm{t}} \times M ; M_{1}\left(Z_{\mathrm{lt}}\right) \times S\right.$ ] be the manifold with corners obtained from $Z_{\text {lt }} \times M$ by blowing up the submanifolds $H \times S$, for all $H \in M_{1}\left(Z_{1 \mathrm{t}}\right)$, in order of decreasing degree; i.e. the codimension of the face $F \in M^{\prime}(Z)$ from which $H$ arises. There are no ordering ambiguities because all hypersurfaces of $Z_{\mathrm{lt}}$ of a given degree are disjoint.

Lemma 2.11. Let $f: X \rightarrow Y$ be a simple b-fibration and $S \subset M$ a $p$ submanifold. Then the map $f_{\mathrm{lt}} \times \mathrm{Id}: X_{\mathrm{lt}} \times M \longrightarrow Y_{\mathrm{lt}} \times M$ lifts to a simple b-fibration

$$
\left[X_{\mathrm{lt}} \times M ; M_{1}\left(X_{\mathrm{lt}}\right) \times S\right] \longrightarrow\left[Y_{\mathrm{lt}} \times M ; M_{1}\left(Y_{\mathrm{lt}}\right) \times S\right] .
$$

Proof. We can argue as in the proof of Lemma 2.8 to reduce the proof to the case where $f$ is of the form (2.26). Now applying Lemma 2.5 to the $M_{1}(Y) \times S$ blowups allows the b-fibration to be lifted and so gives the result. 
Consider the inverse images of all the submanifolds $H \times S$ for all hypersurfaces $H$ of $Y$ of fixed degree $d$. Each such submanifold has as inverse image a union of submanifolds $G \times S$, which are disjoint if they correspond to different $H$. Since $f_{\mathrm{lt}}$ is a b-fibration and $\operatorname{dim} X_{\mathrm{lt}}=\operatorname{dim} Y_{\mathrm{lt}}+1$, we have $d(G)=d(H)$ or $d(H)+1$. Hence in $X_{1 \mathrm{t}} \times M$ we can choose the order of blowup so that the $G \times S$ are also blown up in order of decreasing $d(G)$. Condition (2.2) for $f_{1 \mathrm{t}}$ means that as $H$ runs through all hypersurfaces of $Y_{\text {lt }}, G$ runs through all hypersurfaces of $X_{1 \mathrm{t}}$, so we obtain precisely $\left[X_{\mathrm{lt}} \times M ; M_{1}(X) \times S\right]$ as the new domain.

\section{The Single Space.}

In section 1.4 we noted that the surgery spaces of Part I will not suffice to construct the resolvent of $\Delta$ when the boundary Dirac operator is not invertible. Instead, consideration of the eigenfunctions on an interval under surgery suggests that the resolvent will be a smooth (conormal) function of $y, y^{\prime}$ and the 'rescaled distance'

$$
\frac{\operatorname{arclength}}{\text { total length }}=\frac{\operatorname{arcsinh} \frac{x}{\epsilon}}{2 \operatorname{arcsinh} \frac{1}{\epsilon}} \sim \frac{\operatorname{ilg} \epsilon}{\operatorname{ilg} \frac{\epsilon}{x}} \text { as } \frac{\epsilon}{x} \rightarrow 0 .
$$

\subsection{Definition.}

This suggests working on a space on which these functions are smooth. We therefore make the following definition in terms of the blow up notation introduced in the previous section.

Definition 3. The logarithmic single surgery space is:

$$
X_{\mathrm{Ls}}=\left(X_{s}\right)_{\mathrm{lt}}=\left(\left(X_{s}\right)_{\log }\right)_{\mathrm{tb}} .
$$

Here the single surgery space defined in [21] and Part I is obtained by blow up of $H$ at $\epsilon=0$ :

$$
X_{s}=\left[M \times\left[0, \epsilon_{0}\right] ; H \times\{0\}\right] .
$$

By Lemma 2.8, the b-fibration $X_{s} \longrightarrow\left[0, \epsilon_{0}\right]$ lifts to a b-fibration $X_{\mathrm{Ls}} \longrightarrow\left[0, \text { ilg } \epsilon_{0}\right]_{\mathrm{ilg} \epsilon} \epsilon$. Therefore ilg $\epsilon$ is a smooth function on $X_{\mathrm{Ls}}$, vanishing to first order on all boundary faces $\left(\right.$ at $\epsilon=0$ ). We will understand $X_{\mathrm{Ls}}^{0}$, 
'zero space', to mean $\left[0, \text { ilg } \epsilon_{0}\right]_{\mathrm{ilg} \epsilon}$ below. The space $X_{\mathrm{Ls}}$ has four types of boundary hypersurfaces, cf. Figure 1 . The lift of the boundary $\epsilon=0$ will be denoted $B_{0}\left(X_{\mathrm{Ls}}\right)$; it is the surgery boundary. The lift of the surgery front face will be denoted $B_{1}\left(X_{\mathrm{Ls}}\right)$ and again called the surgery front face. The new hypersurfaces constructed in the last, total boundary, blow up in (3.1) will be called the logarithmic surgery faces and denoted $B_{2}\left(X_{\mathrm{Ls}}\right)$. Defining functions for these space will sometimes be denoted $\rho_{0}, \rho_{1}$, and $\rho_{2}$, respectively. There is also a 'trivial' boundary hypersurface at $\epsilon=\epsilon_{0}$. Under the general assumption that $H$ separates, both $B_{0}\left(X_{\mathrm{Ls}}\right)$ and $B_{2}\left(X_{\mathrm{Ls}}\right)$ have two components; these will be denoted $B_{ \pm 0}\left(X_{\mathrm{Ls}}\right), B_{ \pm 2}\left(X_{\mathrm{Ls}}\right)$ with the sign corresponding to the local orientation of $H$.

The diffeomorphism types of these boundary hypersurfaces are easily identified. Clearly

$$
B_{0}\left(X_{\mathrm{Ls}}\right) \cong \bar{M}_{\log }
$$

is just the manifold with boundary, $\bar{M}$, obtained by cutting $M$ along $H$ with its boundary blown up logarithmically. The front face of $X_{s}$ is the radial compactification of the normal bundle to $H$ in $M$; this compactification is denoted $\bar{H}$. Lifted to $\left(X_{s}\right)_{\log }$ this becomes $\bar{H}_{\text {log }}$. The final blow up does not change the structure of this face so

$$
B_{1}\left(X_{\mathrm{Ls}}\right) \cong \bar{H}_{\log }
$$

The essentially new faces introduced by the passage from $X_{s}$ to $X_{\mathrm{Ls}}$ are the boundaries of the radial compactifications of the normal bundles to the corners of $\left(X_{s}\right)_{\log }$. These are interval bundles over $H$. The two functions $\operatorname{ilg} x$ and $\operatorname{ilg}(\epsilon / x)$ can be taken as defining functions for the corner, in the closure of the region $\epsilon<\frac{1}{2} x<\frac{1}{4}$. Thus the limiting value

$$
s=\left\{\begin{array}{lcc}
\lim _{\mathrm{ilg} x, \mathrm{ilg}(\epsilon / x) \downarrow 0} & \frac{\mathrm{ilg} x}{\mathrm{ilg} x+\mathrm{ilg}(\epsilon / x)}=\frac{\mathrm{ilg} \epsilon}{\mathrm{ilg}(\epsilon / x)} & x>0 \\
\lim _{\mathrm{ilg}-x, \operatorname{ilg}(-\epsilon / x) \downarrow 0} \frac{-\operatorname{ilg}(-x)}{\operatorname{ilg}(-x)+\operatorname{ilg}(-\epsilon / x)}=-\frac{\mathrm{ilg} \epsilon}{\operatorname{ilg}(-\epsilon / x)} & x<0
\end{array}\right.
$$

is a global variable along the fibres. If $x$ is replaced by another defining function for $H, x^{\prime}=a(x, y) x$ with $a>0$, then

$$
\operatorname{ilg} x^{\prime}=\operatorname{ilg} x \frac{1}{1-\operatorname{ilg} x \cdot \log a}, \operatorname{ilg}\left(\epsilon / x^{\prime}\right)=\operatorname{ilg}(\epsilon / x) \frac{1}{1+\mathrm{ilg}(\epsilon / x) \cdot \log a} .
$$




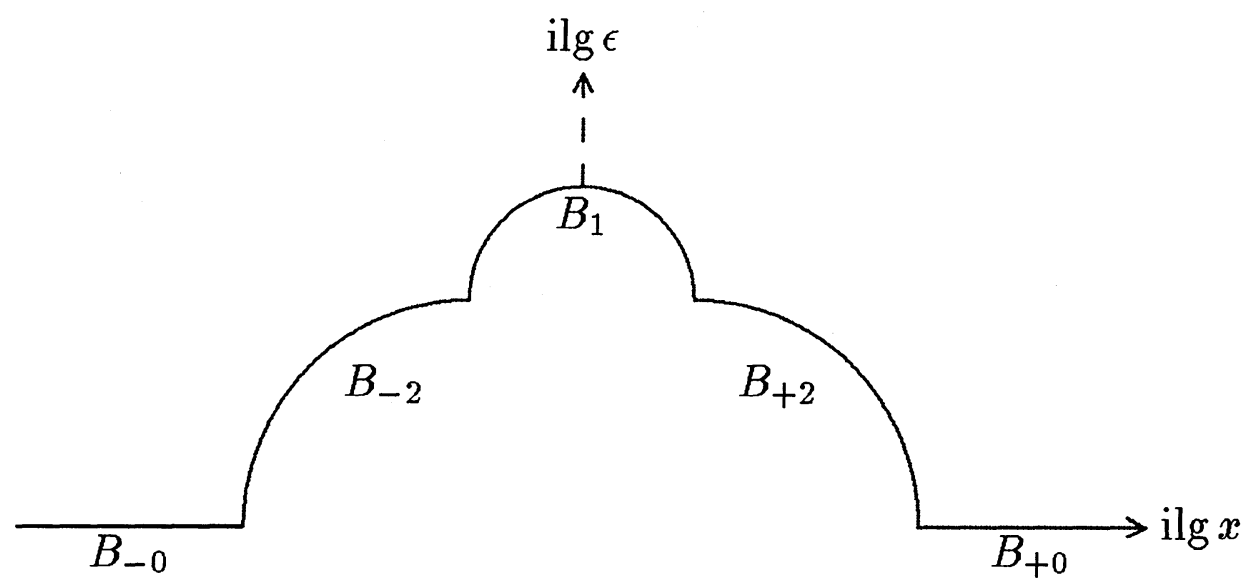

Figure 1.

Thus the limiting value of $s$ in (3.5) is unchanged. It follows that $B_{2}\left(X_{\mathrm{Ls}}\right)$ is a canonically trivial bundle over $H$. Occasionally we will use the notation $\widetilde{H}$ for $\partial \bar{M}=H \sqcup H$, the disjoint union of two copies of $H$, and write $\widetilde{H} \times[0,1]_{s}$ for $H \times\left([-1,0]_{s} \cup[0,1]_{s}\right)$.

\subsection{Densities.}

The Riemannian density of $g_{\epsilon}$ is of the form $\nu_{g}=\left(x^{2}+\epsilon^{2}\right)^{-\frac{1}{2}} \nu$ where $0<$ $\nu \in C^{\infty}\left(M \times\left[0, \epsilon_{0}\right] ; \Omega(M)\right)$. We shall adopt a slightly different normalization of densities from that used in Part I and consider the (trivial) bundle, $\Omega_{X}$, over $M \times\left[0, \epsilon_{0}\right]_{\epsilon}$ which has as generating section $\nu_{g} \otimes|d \epsilon| / \epsilon$. This bundle is just the density bundle over $M \times\left(0, \epsilon_{0}\right]$ and lifts to $X_{s}$ to

$$
(\beta[X,\{0\} \times H])^{*} \Omega_{X} \equiv \Omega_{b}\left(X_{s}\right) .
$$

Notice that if the extra factor of $\epsilon^{-1}$ is omitted, as it is in the normalization of Part I, then one simply gets the density bundle in (3.7) instead of the b-density bundle.

The advantage of the extra factor of $\epsilon^{-1}$ is that $\Omega_{X}$ lifts to be simple on $X_{\text {Ls }}$. By Lemma 2.10 , we have

$$
\beta_{\mathrm{lt}}{ }^{*} \Omega_{b}\left(X_{s}\right)=\Omega_{D}\left(X_{\mathrm{Ls}}\right)=\rho_{0}^{-2} \rho_{2}^{-3} \rho_{1}^{-2} \Omega\left(X_{\mathrm{Ls}}\right) .
$$

Note that the two functions ias $\epsilon$ (see section 1.3) and ilg $\epsilon$ are smooth functions of each other and equal to first order. In view of the behaviour of 
the 'reduced normal operator' of section 5 , it will often be advantageous to use ias $\epsilon$, and we shall frequently do this from now on.

\subsection{Lift of $\mathcal{V}_{s}(X)$.}

In Part I it is shown that the Laplacian associated to a surgery metric lifts to $X_{s}$ to an element of $\operatorname{Diff}_{s}^{2}\left(X_{s}\right)$, which is the enveloping algebra of $\mathcal{V}_{s}\left(X_{s}\right)$. Here $\mathcal{V}_{s}\left(X_{s}\right)$ is the Lie subalgebra of $\mathcal{V}_{b}\left(X_{s}\right)$ consisting of those vector fields which are tangent to the fibres $\{\epsilon=$ const $\}$, it may also be described as consisting of the $C^{\infty}$ vector fields on $X_{s}$ tangent to the fibres and of finite length with respect to $g_{\epsilon}$. We need to consider the lift of $\mathcal{V}_{s}\left(X_{s}\right)$ to $X_{\mathrm{Ls}}$.

As noted in section 3.1, ilg $\epsilon$ lifts to a $C^{\infty}$ function on $X_{\mathrm{Ls}}$ which is a total boundary defining function for all the boundary hypersurfaces above $\epsilon=0$. Consider the Lie algebra

$$
\mathcal{V}_{\mathrm{Ls}}\left(X_{\mathrm{Ls}}\right)=\left\{V \in \mathcal{V}_{b}\left(X_{\mathrm{Ls}}\right) ; V \cdot \operatorname{ilg} \epsilon=0\right. \text { and }
$$

$V$ is tangent to the fibres of $B_{2}\left(X_{\mathrm{Ls}}\right)$ over $\left.[-1,1]\right\}$.

As with $\mathcal{V}_{s}, \mathcal{V}_{\mathrm{Ls}}$ is the space of $C^{\infty}$ sections of a vector bundle. To describe this bundle directly on $X_{\mathrm{Ls}}$, let ${ }^{b} T X_{\mathrm{Ls}}^{\prime} \subset{ }^{b} T X_{\mathrm{Ls}}$ be the subbundle of codimension one given by

$$
{ }^{b} T X_{\mathrm{Ls}}^{\prime}=\operatorname{Ker}\left({ }^{b} d \operatorname{ilg} \epsilon\right):{ }^{b} T X_{\mathrm{Ls}} \rightarrow{ }^{b} T X_{\mathrm{Ls}}^{0} .
$$

Since the map ilg $\epsilon: X_{\mathrm{Ls}} \rightarrow X_{\mathrm{Ls}}^{0}$ is a b-fibration, $\operatorname{Ker}\left({ }^{b} d \operatorname{ilg} \epsilon\right)$ has codimension one at every point. Let $F \subset{ }^{b} T_{B_{2}} X_{\mathrm{Ls}}^{\prime}$ be the subbundle of vectors tangent to the fibres of the fibration $B_{2} \rightarrow[-1,1]$. Then $\mathcal{V}_{\mathrm{Ls}}$ is the space of smooth sections of ${ }^{b} T X_{\mathrm{Ls}}^{\prime}$ that take values in $F$ over $B_{2}$. As explained in [22], section 8, this means that there is a bundle ${ }^{\mathrm{Ls}} T X_{\mathrm{Ls}}={ }^{F}\left({ }^{b} T X_{\mathrm{Ls}}^{\prime}\right)$ (in the notation of [22]) such that $\mathcal{V}_{\mathrm{Ls}}$ is precisely the space of smooth sections of ${ }^{\mathrm{Ls}} T X_{\mathrm{Ls}}$. The metric lifts to a non-degenerate fibre metric on ${ }^{\mathrm{Ls}} T X_{\mathrm{Ls}}$.

Following the approach outlined in [25], the bundle ${ }^{\mathrm{Ls}} T X_{\mathrm{Ls}}$ is taken as the replacement for the usual tangent bundle $T X_{\mathrm{Ls}}$ in surgery geometry. We define surgery form bundles, surgery Clifford bundles, surgery frame bundles and surgery spinor bundles using ${ }^{\mathrm{Ls}} T X_{\mathrm{Ls}}$. Thus the surgery cotangent bundle, ${ }^{\mathrm{Ls}} T^{*} X_{\mathrm{Ls}}$ is defined to be the dual of ${ }^{\mathrm{Ls}} T X_{\mathrm{Ls}}$. The surgery form bundle ${ }^{\mathrm{Ls}} \Lambda^{*} X_{\mathrm{Ls}}$ is the exterior bundle of the surgery cotangent bundle. The surgery Clifford bundle ${ }^{\mathrm{Ls}} \mathrm{Cl} X_{\mathrm{Ls}}$ is the fibrewise Clifford algebra of the surgery cotangent bundle with respect to the fibre metric $g_{\epsilon}$ (defined on ${ }^{\mathrm{Ls}} T^{*} X_{\mathrm{Ls}}$ by duality). If $M^{n}$ is spin, then the bundle of orthonormal frames 
of ${ }^{\mathrm{Ls}} T^{*} X_{\mathrm{Ls}}$ lifts to a $\operatorname{Spin}(n)$ bundle $\operatorname{Spin}\left(X_{\mathrm{Ls}}\right)$, which reduces over $B_{1}$ and $B_{2}$ to have structure group $\operatorname{Spin}(n-1)$. The surgery spinor bundle is the associated bundle

$$
S\left(X_{\mathrm{Ls}}\right)=\operatorname{Spin}\left(X_{\mathrm{Ls}}\right) \times_{\operatorname{Spin}(n)} S,
$$

where $S$ is the spin representation of $\operatorname{Spin}(n)$. If $M$ is odd-dimensional then the spin representation is irreducible on $\mathbb{C}^{2^{k}}$ with $n=2 k+1$ and over $B_{2}, S\left(X_{\mathrm{Ls}}\right)$ has a natural splitting $S\left(X_{\mathrm{Ls}}\right)=S^{+}(H) \oplus S^{-}(H)$ given by the \pm 1 eigenspaces for homomorphism given by multiplication by the surgery Clifford element $d x /\left(\sqrt{x^{2}+\epsilon^{2}}\right)$. Restricted to each of the leaves of $B_{2}$ on which $s$ is constant they are the plus and minus spinor spaces, associated with the induced spin structure on $H$, on which the Dirac operator $\partial_{H}$ acts.

The space of Ls-differential operators, $\operatorname{Diff}_{\mathrm{Ls}}^{*}\left(X_{\mathrm{Ls}} ; E, F\right)$, (where we write $\left.\operatorname{Diff}_{\mathrm{Ls}}^{*}\left(X_{\mathrm{Ls}} ; E, E\right)=\operatorname{Diff}_{\mathrm{Ls}}^{*}\left(X_{\mathrm{Ls}} ; E\right)\right)$ consists of those differential operators from sections of $E$ to sections of $F$ which are sums of products of vector fields in $\mathcal{V}_{\mathrm{Ls}}$, acting via some connection, and smooth bundle maps (in other words, the enveloping algebra of $\mathcal{V}_{\mathrm{Ls}}$ tensored with bundle maps).

Lemma 3.1. If $\delta$ is the adjoint of $d$ with respect to $g_{\epsilon}$, then

$$
d+\delta \in \operatorname{Diff}_{\mathrm{Ls}}^{1}\left(X_{\mathrm{Ls}} ;{ }^{\mathrm{Ls}} \Lambda^{*} X_{\mathrm{Ls}}\right)
$$

and

$$
\Delta_{\epsilon}=(d+\delta)^{2} \in \operatorname{Diff}_{\mathrm{Ls}}^{2}\left(X_{\mathrm{Ls}} ;{ }^{\mathrm{Ls}} \Lambda^{*} X_{\mathrm{Ls}}\right) .
$$

The Dirac operator for a Hermitian Clifford connection on an Hermitan ${ }^{\mathrm{Ls}} T^{*} X_{\mathrm{Ls}}$-Clifford module, $E$, is an element ${ }_{\epsilon} \in \operatorname{Diff}_{\mathrm{Ls}}^{1}\left(X_{\mathrm{Ls}} ; E\right)$.

Proof. We may write $\partial_{\epsilon}=\sum_{i} \operatorname{cl}\left(e_{i}\right) \nabla_{i}$, for an orthonormal frame $e_{i}$ of the surgery cotangent bundle; directly from the definition this shows $\tilde{\partial}_{\epsilon}$ to be a first order Ls-differential operator. Similarly, we may write $d=\sum_{i}\left(e_{i} \wedge\right) \nabla_{i}$ and $\delta=-\sum_{i} \nabla_{i}\left(e_{i}\llcorner)\right.$, and $\left(e_{i} \wedge\right)$ and $e_{i}$ L are smooth bundle maps on ${ }^{\mathrm{Ls}} \Lambda^{*} X_{\mathrm{Ls}}$. Hence, $\Delta_{\epsilon}=(d+\delta)^{2}$ is also a Ls-differential operator.

In particular this applies to 'the' Dirac operator on spinors if $M$ is spin. The next result identifies $\mathcal{V}_{\mathrm{Ls}}$ in terms of the Lie algebra $\mathcal{V}_{s}$ of Part I:

Lemma 3.2. The Lie algebra $\mathcal{V}_{s}\left(X_{s}\right)$ lifts to $X_{\mathrm{Ls}}$ to span, over $C^{\infty}\left(X_{\mathrm{Ls}}\right)$, the boundary-fibration structure $\mathcal{V}_{\mathrm{Ls}}\left(X_{\mathrm{Ls}}\right)$ given by (3.9). 
Proof. Away from the blown up submanifolds this is obviously so. Moreover it is certainly local over open sets of $M$ so it suffices to consider the product case $M=H \times(-1,1)$ where $H$ is just an open set in Euclidean space. The blow-ups then preserve the product structure so it suffices to consider the case that $H$ is a point. Thus we only need consider the lift of the vector field

$$
V_{0}=\left(x^{2}+\epsilon^{2}\right)^{\frac{1}{2}} \frac{\partial}{\partial x} .
$$

Near the corner of $X_{s}$ the projective coordinates $k=\epsilon / x$ and $x$ are valid and in terms of these

$$
V_{0}=\left(1+k^{2}\right)^{\frac{1}{2}}\left(x \frac{\partial}{\partial x}-k \frac{\partial}{\partial k}\right) .
$$

Under the logarithmic blow up of both boundary hypersurfaces this in turn lifts to

$$
V_{0}=\left(1+\exp \left(-\frac{2}{\kappa}\right)\right)^{\frac{1}{2}}\left(\xi^{2} \frac{\partial}{\partial \xi}-\kappa^{2} \frac{\partial}{\partial \kappa}\right)
$$

where $\xi=\operatorname{ilg} x$ and $\kappa=$ ilg $k$. Finally under the radial blow up of $\xi=\kappa=0$ this becomes

$$
V_{0}=\left(1+\exp \left(-\frac{2\left(1-\rho_{0}\right)}{\rho_{2}}\right)\right)^{\frac{1}{2}}\left(\rho_{2}^{2} \frac{\partial}{\partial \rho_{2}}-\rho_{0} \rho_{2} \frac{\partial}{\partial \rho_{0}}\right)
$$

or

$$
V_{0}=\left(1+\exp \left(-\frac{2}{\rho_{2}}\right)\right)^{\frac{1}{2}}\left(\rho_{2}^{2} \frac{\partial}{\partial \rho_{2}}-\rho_{1} \rho_{2} \frac{\partial}{\partial \rho_{1}}\right)
$$

in terms of the coordinates $\rho_{2}=\xi$ and $\rho_{0}=\kappa /(\kappa+\xi)=(1-s)$ or $\rho_{2}=\kappa$ and $\rho_{1}=\xi /(\kappa+\xi)=s$ which together cover the new face. It is now easy to check that $V_{0}$ spans the algebra $\mathcal{V}_{\mathrm{Ls}}\left(X_{\mathrm{Ls}}\right)$ in this case, so proving the result in general.

This Lemma also shows that ${ }^{\mathrm{L} s} T X_{\mathrm{Ls}} \equiv \beta_{\mathrm{lt}}{ }^{*}\left({ }^{s} T X_{s}\right)$, where ${ }^{s} T X_{s}$ is the surgery tangent space of Part I.

Consider the normal operators for the structure $\mathcal{V}_{\mathrm{Ls}}\left(X_{\mathrm{Ls}}\right)$. At the level of the Lie algebra these correspond to freezing the coefficients of $\mathcal{V}_{\mathrm{Ls}}\left(X_{\mathrm{Ls}}\right)$ at the various integral submanifolds. Each level surface of $\epsilon$ on which it is nonzero forms such an integral submanifold and $\mathcal{V}_{\mathrm{Ls}}\left(X_{\mathrm{Ls}}\right)$ restricts to the space of all smooth vector fields on these surfaces. The more interesting integral 
submanifolds are those lying over $\epsilon=0$. There are three essentially different cases. Consider first the restriction algebras. Let us use the notation $\mathcal{V}_{f}(Y)$ for the subalgebra of $\mathcal{V}_{b}(Y)$ consisting of those vector fields tangent to the leaves of a b-fibration $f$ and $\mathcal{V}_{c}(X)$ for the cusp algebra, determined by a choice of boundary defining function $\rho$ on a compact manifold $X$ with boundary $Y$ :

$$
\mathcal{V}_{c}(X)=C^{\infty}(X)-\operatorname{span}\left\{\rho^{2} \frac{\partial}{\partial \rho}, \frac{\partial}{\partial y_{i}}\right\}
$$

Lemma 3.3. Restriction to the three types of boundary hypersurfaces above $\epsilon=0$ gives surjective Lie algebra homomorphisms

$$
\begin{gathered}
R_{0}=N_{0}: \mathcal{V}_{\mathrm{Ls}}(X) \longrightarrow \mathcal{V}_{c}\left(\bar{M}_{\mathrm{log}}\right) \\
R_{2}: \mathcal{V}_{\mathrm{Ls}}(X) \longrightarrow \mathcal{V}_{f}(\widetilde{H} \times[0,1]) \\
R_{1}=N_{1}: \mathcal{V}_{\mathrm{Ls}}(X) \longrightarrow \mathcal{V}_{c}\left(\bar{H}_{\mathrm{log}}\right)
\end{gathered}
$$

where $f: \widetilde{H} \times[0,1] \longrightarrow[0,1]$ is the projection.

Proof. As in the proof of Lemma 3.2 it suffices to consider the special case where $M$ is an interval. Then (3.13) just arises as the restriction of $V_{0}$ in (3.11) to $s=0$, giving $\xi^{2} \partial / \partial \xi$, which, with the vector fields on $H$, generates the cusp algebra with distinguished boundary defining function $\xi$. The other two restriction maps can be analyzed in the same way, with (3.14) arising from the fact that $V_{0}$ in (3.12) vanishes at $\rho_{2}=0$.

The notation $N_{0}$ and $N_{1}$ in (3.13) and (3.15) is justified by the fact that the null spaces of these maps are precisely the Lie ideals $\mathcal{I} \cdot \mathcal{V}_{\mathrm{Ls}}(X)$ where $\mathcal{I}$ is the ideal of functions defining the boundary hypersurface in question; thus these maps are indeed the normal operators in the sense of [23] and the range spaces can be identified with $C^{\infty}\left(H ;{ }^{\mathrm{Ls}} T X_{\mathrm{Ls}}\right)$ for the corresponding boundary hypersurface in $M_{1}\left(X_{\mathrm{Ls}}\right)$.

This is not the case for restriction to $B_{2}\left(X_{\mathrm{Ls}}\right)$. Rather, the null space of the restriction map

$$
C^{\infty}\left(B_{2}\left(X_{\mathrm{Ls}}\right) ;{ }^{\mathrm{Ls}} T X_{\mathrm{Ls}}\right) \longrightarrow \mathcal{V}_{f}(\tilde{H} \times[0,1])
$$

is a one-dimensional Lie algebra over $B_{2}\left(X_{\mathrm{Ls}}\right)$. This line bundle is important since it generates the reduced normal operator, the properties of which largely determine the behaviour of the small eigenvalues of the Dirac operator and Laplacian. In fact 
Lemma 3.4. If $V \in \mathcal{V}_{\mathrm{Ls}}(X)$ has $R_{2}(V)=0$ then $V / \mathrm{ilg} \epsilon$ is smooth up to the interior of $B_{2}\left(X_{\mathrm{Ls}}\right)$ and, by projecting off any term in $\mathcal{V}_{f}(\widetilde{H} \times[0,1])$, defines a vector field

$$
\mathrm{RN}(V)=\operatorname{rn}(V) D_{s}, \text { with } \operatorname{rn}(V) \in C^{\infty}\left(B_{2}\right),
$$

on the fibres of $B_{2}\left(X_{\mathrm{Ls}}\right)$ over $\widetilde{H}$; this vector field is smooth up to the boundary (but not necessarily vanishing there) and is such that $\operatorname{rn}(V)=0$ if and only $V \in \mathcal{I}\left(B_{2}\right) \cdot \mathcal{V}_{\mathrm{Ls}}(X)$.

Proof. Again this is just a matter of examining the behaviour of the vector field $V_{0}$, in (3.11). Using the coordinates of (3.11), in the interior of $B_{2}\left(X_{\mathrm{Ls}}\right)$ ilg $\epsilon=\rho_{1} \rho_{2}$ so the restriction of (ilg $\left.\epsilon\right)^{-1} V_{0}$ to $\xi=0$ is just

$$
\mathrm{RN}\left(V_{0}\right)=\frac{\partial}{\partial s} \text {. }
$$

This shows it to be smooth and non-vanishing up to both boundaries of $B_{2}$.

Thus the two maps $R_{2}$ and RN together capture the full normal operator at $B_{2}\left(X_{\mathrm{Ls}}\right)$.

Let us now calculate quite explicitly the form of the Dirac operator and the Laplacian in local coordinates near the boundary hypersurfaces of $X_{\mathrm{Ls}}$. Choose a product decomposition of a neighbourhood of $H \subset M$ and let $(x, y)$ be corresponding coordinates, where $y$ denotes local coordinates on $H$. Write $p$ for local coordinates on $M$.

Lemma 3.5. We have the following expressions in coordinates for the Dirac operator and the Laplacian near the boundary hypersurfaces of $X_{\mathrm{Ls}}$ :

(i) Near $B_{1}$, and away from $B_{2}$, using coordinates $y, r=\sinh ^{-1}(x / \epsilon)$ and $\operatorname{ias} \epsilon$, we have

$$
\begin{aligned}
\check{\partial}_{\epsilon} & =\gamma\left(-i \nabla_{r}\right)+\check{\partial}_{H}+v \cdot Q, \\
\Delta_{\epsilon} & =-\left(\nabla_{r}\right)^{2}+\Delta_{H}+v \cdot Q^{\prime}
\end{aligned}
$$

where $v$ is a function vanishing to infinite order at this face and $Q, Q^{\prime}$ are Ls-differential operators of order at most one, respectively two. have

$$
\begin{aligned}
ð_{\epsilon} & =\operatorname{ias} \epsilon \cdot \gamma\left(-i \nabla_{s}\right)+\partial_{H}+v \cdot Q, \\
\Delta_{\epsilon} & =-(\operatorname{ias} \epsilon)^{2}\left(\nabla_{s}\right)^{2}+\Delta_{H}+v \cdot Q^{\prime}
\end{aligned}
$$


with $v$, a function vanishing to infinite order at $B_{2}$ and $Q, Q^{\prime}$ as above.

(iii) Near $B_{0}$, using coordinates $p$ and ias $\epsilon$,

$$
\begin{aligned}
\partial_{\epsilon} & =\succsim_{0}+v \cdot Q, \\
\Delta_{\epsilon} & =\Delta_{0}+v \cdot Q^{\prime}
\end{aligned}
$$

with $v$ a function vanishing to infinite order at $B_{0}$ and $Q, Q^{\prime}$ as above.

Proof. If $h$ in the definition of the surgery metric is a product metric in $|x|<\delta$ for some defining function $x$ for $H$, then (3.18), (3.19) follow easily from $(3.10)-(3.12)$. So write

$$
\begin{aligned}
h & =h_{i j}(0, y) d y^{i} d y^{j}+x \cdot h_{i j}^{\prime}(x, y) d y^{i} d y^{j}+h_{j}^{\prime \prime}(x, y) d x d y_{j}+h^{\prime \prime \prime}(x, y) d x^{2} \\
& =h_{i j}(0, y) d y^{i} d y^{j}+x \cdot h_{i j}^{\prime}(x, y) d y^{i} d y^{j} \\
& +\sqrt{x^{2}+\epsilon^{2}} h_{j}^{\prime \prime}(x, y) \frac{d x}{\sqrt{x^{2}+\epsilon^{2}}} d y_{j}+\left(x^{2}+\epsilon^{2}\right) h^{\prime \prime \prime}(x, y) \frac{d x^{2}}{\left(x^{2}+\epsilon^{2}\right)}
\end{aligned}
$$

giving the metric in terms of the smooth surgery form $d x / \sqrt{x^{2}+\epsilon^{2}}$. The lifts to $X_{\mathrm{Ls}}$ of $x, \sqrt{x^{2}+\epsilon^{2}}$ and $\left(x^{2}+\epsilon^{2}\right)$ vanish to infinite order at $B_{1}$ and $B_{2}$ so only contribute to $\partial$ or $\Delta$ a term of the form $v \cdot Q$ or $v \cdot Q^{\prime}$. The first term is a product metric in a collar neighbourhood of $H$ so gives the principal terms. Hence (3.18) and (3.19) are established. (3.20) follows because in the interior of $B_{0}$, the surgery metric $g_{\epsilon}=g_{0}+v \cdot g^{\prime}$ with $v$ vanishing rapidly on $B_{0}$ and $g^{\prime}$ smooth on $X_{\mathrm{Ls}}$.

\subsection{Models.}

The Lie algebra homomorphisms in (3.13) - (3.15) extend to homomorphisms of the enveloping algebras and define three of the four (closely related) 'model problems' we need to discuss in order to invert the original operator.

Proposition 3.6. For the Dirac operator (Laplacian) of the metric $g_{\epsilon}$ the normal operator $R_{0}\left(\check{\partial}_{\epsilon}\right)\left(R_{0}\left(\Delta_{\epsilon}\right)\right)$ is the lift to $\bar{M}_{\log }$ of the Dirac operator (Laplacian), $\partial_{0}\left(\Delta_{0}\right)$, of $\bar{M}$ for the exact b-metric $g_{0}$; the normal operator $R_{1}\left(\widetilde{\partial}_{\epsilon}\right)\left(R_{1}\left(\Delta_{\epsilon}\right)\right)$ is the lift to $\bar{H}_{\log }$ of the indicial operator of $\check{\partial}_{0}\left(\Delta_{0}\right)$ as an $\mathbb{R}^{+}$-invariant operator in $\bar{H}$, the normal bundle to $H$ in $X$; and the restriction operator $R_{2}\left(\partial_{\epsilon}\right)\left(R_{2}\left(\Delta_{\epsilon}\right)\right)$ is $\partial_{H}\left(\Delta_{H}\right)$, acting on the leaves of $B_{2}\left(X_{\mathrm{Ls}}\right)$. 
Thus the first three model problems are just the Laplacian on $\bar{M}$ and its indicial operator (in two guises). The fourth model problem arises from the reduced normal operator of Lemma 3.4.

Proposition 3.7. Let $\Pi$ be the orthogonal projection onto the null space of $\partial_{H}$ and let $s$ be the coordinate in (3.5). If $u \in C^{\infty}\left(B_{2}\left(X_{\mathrm{Ls}}\right)\right)$ is in the range of $\Pi$ and $\tilde{u}$ is any smooth extension of $u$ to $X_{\mathrm{Ls}}$, then

$$
\mathrm{RN}(ð) u=\Pi\left((\operatorname{ias} \epsilon)^{-1} \partial \tilde{u}\left\lceil B_{2}\left(X_{\mathrm{Ls}}\right)\right)=\gamma D_{s} u,\right.
$$

independent of the choice of extension. Similarly, let $\bar{\Pi}$ be the orthogonal projection on the null space of $\Delta_{H}$, and let $W$ be a vector field normal to $B_{2}$ tangent to $s=y=$ constant. If $u \in C^{\infty}\left(B_{2}\left(X_{\mathrm{Ls}}\right)\right)$ is in the range of $\bar{\Pi}$ and $\tilde{u}$ is an extension of $u$ to $C^{\infty}\left(X_{\mathrm{Ls}}\right)$ such that $\Delta_{H}\left(W \tilde{u}\left\lceil B_{2}\right)=0\right.$, then $\Delta \tilde{u}$ vanishes to second order at $B_{2}$ and

$$
\mathrm{RN}(\Delta) u=\bar{\Pi}\left(\left.(\operatorname{ias} \epsilon)^{-2} \Delta \tilde{u}\right|_{B_{2}}\left(X_{\mathrm{Ls}}\right)\right)=D_{s}^{2} u,
$$

independent of the choice of extension.

Proof. In the interior of $B_{2}$, we can use $s, y$ and ias $\epsilon$ as coordinates. Then, writing $\tilde{u}$ as a Taylor series in ias $\epsilon$ off $B_{2}$ and using the coordinate representations of $ð$ and $\Delta$ in (3.19), the proposition follows.

The fourth model operator is therefore the ordinary differential operator $\gamma D_{s}\left(D_{s}^{2}\right)$ acting on smooth functions on $[-1,0] \cup[0,1]$ with values in the null space of $\partial_{H}\left(\Delta_{H}\right)$. In fact this operator comes with boundary conditions which turn it into an unbounded self-adjoint operator on the interval $[-1,1]$. To discuss these we need to recall, from [22], some properties of the 'extended $L^{2}$ null space' of an elliptic operator. Here the cases of the Dirac operator and the Laplacian are somewhat different, so we discuss them separately. Let us treat the Laplacian first, as it is the more complicated case. Consider the null space of $\Delta_{\bar{M}}$ on the weighted $L^{2}$ space $x^{-\delta} L_{b}^{2}\left(M_{ \pm}\right)$, for $\delta$ small. Each element $v$ of this null space has an asymptotic expansion

$$
\begin{aligned}
\Delta_{\bar{M}} v=0, v \in x^{-\delta} L_{b}^{2}\left(M_{ \pm}\right) \Longrightarrow & v=v_{1} \log x+v_{0}+v^{\prime} \\
& \text { near } H, \Delta_{H}\left(v_{i}\right)=0, v^{\prime} \in x^{\delta} L_{b}^{2} .
\end{aligned}
$$

These generalized boundary values define a map into $\operatorname{Ker}\left(\Delta_{H}\right) \oplus \operatorname{Ker}\left(\Delta_{H}\right)$; the range will be written $\Lambda_{ \pm}$. It is necessarily a Lagrangian subspace 
of $\operatorname{Ker}\left(\Delta_{H}\right) \oplus \operatorname{Ker}\left(\Delta_{H}\right)$, so in particular has dimension equal to that of $\operatorname{Ker}\left(\Delta_{H}\right)$. For $\delta>0$ small enough this gives a short exact sequence

$$
\begin{aligned}
0 \longrightarrow\left\{v \in x^{\delta} L_{b}^{2}\left(M_{ \pm}\right) ; \Delta_{0} v=0\right\} \longrightarrow \\
\longrightarrow\left\{v \in x^{-\delta} L_{b}^{2}\left(M_{ \pm}\right) ; \Delta_{0} v=0\right\} \longrightarrow \Lambda_{ \pm} \longrightarrow 0
\end{aligned}
$$

This Lagrangian subspace defines the boundary condition for the reduced normal operator. From these Lagrangian spaces we define two subspaces of $\operatorname{Ker}\left(\Delta_{H}\right)$ :

$$
\begin{gathered}
\Lambda_{ \pm}^{D}=\left\{u^{\prime} \in \operatorname{Ker}\left(\Delta_{H}\right) ;\left(u^{\prime}, 0\right) \in \Lambda\right\} \\
\Lambda_{ \pm}^{N}=\left\{u^{\prime \prime} \in \operatorname{Ker}\left(\Delta_{H}\right) ; \exists\left(u^{\prime}, u^{\prime \prime}\right) \in \Lambda\right\} .
\end{gathered}
$$

Clearly the sum of the dimensions of $\Lambda_{ \pm}^{D}$ and $\Lambda_{ \pm}^{N}$, is equal, for either sign, to the dimension of $\operatorname{Ker}\left(\Delta_{H}\right)$. It was also shown in [22] that $\Lambda_{ \pm}^{D}$ and $\Lambda_{ \pm}^{N}$ are the \pm 1 eigenspaces of the scattering matrix associated to $\Delta_{M_{ \pm}}$at $\lambda=0$, so the boundary conditions are determined by the scattering matrix.

To understand the boundary conditions associated to the fourth model operator, we prove a lemma concerning approximate small eigenfunctions $u$, by which we will mean $u \in C^{\infty}\left(X_{\mathrm{Ls}} ; \Omega_{D}^{\frac{1}{2}}\left(X_{\mathrm{Ls}}\right)\right)$ such that $\left(\Delta-(\operatorname{ias} \epsilon)^{2} z^{2}\right) u \epsilon$ $\rho_{0}^{2} \rho_{1}^{2} \rho_{2}^{3} C^{\infty}\left(X_{\mathrm{Ls}} ; \Omega_{D}^{\frac{1}{2}}\left(X_{\mathrm{Ls}}\right)\right)$. Here, 'small' refers to the fact that the eigenvalue goes to zero with ias $\epsilon$. We shall see in section 6 that each such $u$ is indeed a good approximation to a surgery eigenfunction. For such $u$ we have by Proposition 3.7, $\Delta_{H}\left(u\left\lceil B_{2}\right)=0\right.$, so $u\left\lceil B_{2}\right.$ can be regarded as a $\operatorname{Ker}\left(\Delta_{H}\right)$ valued function $\bar{u}$ on $[-1,0]_{s} \cup[0,1]_{s}$.

Proposition 3.8. Suppose $u \in C^{\infty}\left(X_{\mathrm{Ls}} ; \Omega_{D}^{\frac{1}{2}}\left(X_{\mathrm{Ls}}\right)\right)$ satisfies

$$
\left(\Delta-(\operatorname{ias} \epsilon)^{2} z^{2}\right) u \in \rho_{0}^{2} \rho_{1}^{2} \rho_{2}^{3} C^{\infty}\left(X_{\mathrm{Ls}} ; \Omega_{D}^{\frac{1}{2}}\left(X_{\mathrm{Ls}}\right)\right) .
$$

Then $\bar{u}$ is $C^{\infty}$ on $[-1,1]_{s}$, with

$$
\begin{aligned}
& \left(D_{s}^{2}-z^{2}\right) \bar{u}=0, \\
& \text { and }\left.\bar{u}\right|_{\{s=-1\}} \in \Lambda_{-}^{D},\left.D_{s} \bar{u}\right|_{\{s=-1\}} \in \Lambda_{-}^{N} \text {, } \\
& \left.\bar{u}\right|_{\{s=+1\}} \in \Lambda_{+}^{D},\left.D_{s} \bar{u}\right|_{\{s=+1\}} \in \Lambda_{+}^{N} .
\end{aligned}
$$

Conversely, if $\bar{u}$ satisfies (3.27) and (3.28) then there is an extension $u$ satisfying (3.26). 
Proof. We first show that $\bar{u}$ and $\partial_{s} \bar{u}$ match across $s=0$. Using the local coordinates ias $\epsilon, r=\sinh ^{-1}(x / \epsilon)$ and $y$ near $B_{1}$, we have by (3.18) $(\Delta-$ $\left.(\text { ias } \epsilon)^{2} z^{2}\right)=-\left(\frac{\partial}{\partial r}\right)^{2}+\Delta_{H}$ plus terms of second order. On $B_{1}, u\left\lceil B_{1}\right.$ is bounded, and $\left(\Delta_{H}-\left(\frac{\partial}{\partial r}\right)^{2}\right)\left(u\left\lceil B_{1}\right)=0\right.$, so $u \uparrow B_{1}$ must be constant in $r$; hence the values of $\bar{u} \uparrow B_{2}$ match across $s=0$. We also have $\left(\Delta_{H}-\right.$ $\left.\left(\frac{\partial}{\partial r}\right)^{2}\right)\left(\left(\partial_{\text {ias } \epsilon} u\right)\left\lceil B_{1}\right)=0\right.$, and $\left(\partial_{\text {ias } \epsilon} u\right)\left\lceil B_{1}=r \cdot\left(\partial_{\rho_{1}} u\right) \in r \cdot C^{\infty}\left(B_{1}\right)\right.$. Thus $\partial_{\text {ias } \epsilon} u$ is linear in $r$ so can be written $\left(\left(\partial_{\text {ias } \epsilon} u\right)\left\lceil B_{1}\right)=\alpha+\beta r\right.$. It should be noted here that we are taking the derivative $\partial_{\text {ias } \epsilon}$ keeping $r$ and $y$ fixed, not lifting $\partial_{\text {ias } \epsilon}$ from $X_{\mathrm{Ls}}^{0}$. Hence

$$
\frac{\partial u}{\partial s} \uparrow B_{+2} \cap B_{1}=\beta=\frac{\partial u}{\partial s} \uparrow B_{-2} \cap B_{1} .
$$

Thus $\partial_{s} u$ also matches across $s=0$.

A similar argument on $B_{2}$ and $B_{0}$ shows (3.28). Since $u\left\lceil B_{0} \in \operatorname{Ker}\left(\Delta_{\bar{M}}\right)\right.$ and is bounded, the boundary value $u\left\lceil B_{0} \cap B_{2} \in \Lambda_{ \pm}^{D}\right.$. Near $B_{0} \cap B_{2}$ we can use coordinates $\xi=\operatorname{ilg} x$, ias $\epsilon$ and $y$. Then by (3.20) $\left(\Delta-(\operatorname{ias} \epsilon)^{2} z^{2}\right)=$ $-\left(\xi^{2} \frac{\partial}{\partial \xi}\right)^{2}+\Delta_{\bar{M}}$ up to terms of second order. We therefore have $\Delta_{\bar{M}}\left(\partial_{\text {ias } \epsilon} u \uparrow\right.$ $\left.B_{0}\right)=0$, and $\left(\partial_{\text {ias } \epsilon} u\right)\left\lceil B_{0}=\xi^{-1}\left(\partial_{s} u\right) \in \xi^{-1} C^{\infty}\left(B_{0}\right)\right.$, so

$$
\frac{\partial u}{\partial s}\left\lceil B_{ \pm 2} \cap B_{0}=\xi \cdot \frac{\partial u}{\partial(\text { ias } \epsilon)}\left\lceil B_{0} \cap B_{2} \in \Lambda_{ \pm}^{N} .\right.\right.
$$

This establishes (3.28). Finally, we show the smoothness of $\bar{u}$. Near the interior of $B_{2}$, using $s$, ias $\epsilon$ and $y$ we have, by (3.19) $\left(\Delta-(\text { ias } \epsilon)^{2} z^{2}\right)=$ $(\text { ias } \epsilon)^{2} \cdot\left(D_{s}^{2}-z^{2}\right)+\Delta_{H}$ up to terms of infinite order. Since $\left(\Delta-(\text { ias } \epsilon)^{2} z^{2}\right) u$ vanishes to third order on $B_{2}$,

$$
\left(D_{s}^{2}-z^{2}\right) \bar{u}=\Delta_{H}\left(\left(\frac{\partial}{\partial(\operatorname{ias} \epsilon)}\right)^{2} u \uparrow B_{2}\right) .
$$

The left hand side is in $\operatorname{Ker}\left(\Delta_{H}\right)$ and the right hand side is orthogonal to the null space, so each must vanish. This gives (3.27). Since $\bar{u}$ satisfies a second order ordinary differential equation and $\bar{u}$ and its derivative match at $s=0, \bar{u}$ extends smoothly across $s=0$. This yields the necessity of (3.27) and (3.28).

To prove the sufficiency, we reverse the argument. The discussion above yields the unique first and second terms in the Taylor series off $B_{0}$ and $B_{1}$, which are compatible with the given $\bar{u}$ and are annihilated by the normal operators $R_{0}(\Delta), R_{1}(\Delta)$. These are also compatible with taking $\partial_{\rho_{2}} u \uparrow B_{2}=$ 0 . Then, by Propositions 3.6 and 3.7, $u$ satisfies (3.26).

The argument to establish the boundary conditions for the Dirac operator is similar, but less complicated since it is a first order operator. In this 
case, we have as with $\Delta$ that if $\delta>0$ is small enough each element of the null space of $\partial_{0}$ on the weighted $L^{2}$ space $x^{-\delta} L_{b}^{2}\left(M_{ \pm}\right)$has a decomposition near the boundary

$$
\begin{aligned}
\partial_{0} v=0, v \in x^{-\delta} L_{b}^{2}\left(M_{ \pm}\right) \Longrightarrow \\
v(x, y)=v_{0}(y)+v^{\prime}(x, y), \partial_{H}\left(v_{0}\right)=0, v^{\prime} \in x^{\delta} L_{b}^{2}\left(M_{ \pm}\right) .
\end{aligned}
$$

The boundary value $v_{0}$ defines a map into $\operatorname{Ker}\left(\coprod_{H}\right)$; let us call the range $\Lambda_{\tilde{\delta}, \pm}$. It is a Lagrangian subspace of $\operatorname{Ker}\left(\partial_{\tilde{H}}\right)$ with respect to the symplectic structure on $\operatorname{Ker}\left(\partial_{\tilde{H}}\right)$ given by the operator $\gamma=\operatorname{cl}\left(d x /\left(\sqrt{x^{2}+\epsilon^{2}}\right)\right.$. Then we have for the Dirac operator a result analogous to Proposition 3.8:

Proposition 3.9. Suppose $u \in C^{\infty}\left(X_{\mathrm{Ls}}\right)$ satisfies $\left(\coprod_{\epsilon}-(\operatorname{ias} \epsilon) z\right) u=0$. Then $\bar{u}$ is $C^{\infty}$ on $[-1,1]_{s}$, with

$$
\begin{gathered}
\left(\gamma D_{s}-z\right) \bar{u}=0, \\
\left.\bar{u}\right|_{\{s=-1\}} \in \Lambda_{\widetilde{\delta},-} \\
\text { and }\left.\bar{u}\right|_{\{s=+1\}} \in \Lambda_{\check{\partial,+} \cdot}
\end{gathered}
$$

The proof proceeds completely analogously to the first part of the proof of Proposition 3.8, so it is omitted.

\section{The double space and the pseudodifferential calculus.}

\subsection{Preliminary remarks.}

In the previous section, Ls-differential operators were defined. In this section, we set up a calculus of pseudodifferential operators adapted to 'surgery geometry', in the expectation, justified below, that the generalized inverses of elliptic Ls-differential operators will lie in the calculus.

Our strategy for constructing the resolvent of $\Delta_{\epsilon}$ is:

(i) Construct a parametrix $G$ such that

$$
\left(\Delta_{\epsilon}-\lambda^{2}\right) G=\mathrm{Id}-R,
$$

where $\mathrm{R}$ is a 'small' remainder (residual operator).

(ii) Invert $\mathrm{Id}-R$ using the Neumann series:

$$
(\mathrm{Id}-R)^{-1}=\mathrm{Id}+R+R^{2}+\cdots=\mathrm{Id}+S .
$$


(iii) Write the resolvent as

$$
\left(\Delta_{\epsilon}-\lambda^{2}\right)^{-1}=G \cdot(\operatorname{Id}+S) .
$$

To carry out this program, we need to identify a space (an ideal) of residual operators which 'iterate away', that is, such that the Neumann series makes sense, is summable and sums to an element of our calculus. We also need to understand the compositions $\Delta_{\epsilon} \cdot G$, although this is easy since $\Delta_{\epsilon}$ is a differential operator, and $G \cdot S$. In the rest of this section we proceed to set up this machinery to carry through these computations.

\subsection{Logarithmic Double space.}

By analogy with the logarithmic single space, define

$$
X_{\mathrm{Ls}}^{2}=\left(X_{s}^{2}\right)_{\mathrm{lt}}=\left(\left(X_{s}^{2}\right)_{\log }\right)_{\mathrm{tb}} .
$$

Recall that the surgery double space of Part I is

$$
X_{s}^{2}=\left[X^{2} \times\left[0, \epsilon_{0}\right] ; H^{2} \times\{0\} ; X \times H \times\{0\} ; H \times X \times\{0\}\right] .
$$

By Lemma 2.8 , the b-fibrations $\pi_{s, L}^{2}$ and $\pi_{s, R}^{2}: X_{s}^{2} \longrightarrow X_{s}$ lift to bfibrations $\pi_{\mathrm{Ls}, L}^{2}$ and $\pi_{\mathrm{Ls}, R}^{2}: X_{\mathrm{Ls}}^{2} \longrightarrow X_{\mathrm{Ls}}$, such that the following diagram commutes:

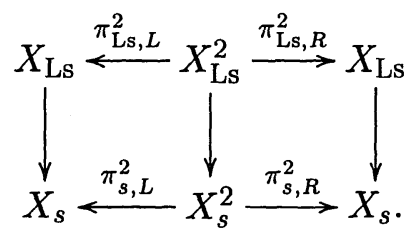

The lifted diagonal $\Delta_{\mathrm{Ls}} \subset X_{\mathrm{Ls}}^{2}$ is a closed p-submanifold which is canonically diffeomorphic to $X_{\mathrm{Ls}}$; indeed, both projections $\pi_{s, L}^{2}, \pi_{s, R}^{2}$ restrict to give the same diffeomorphism from $\Delta_{\mathrm{Ls}}$ to $X_{\mathrm{Ls}}$. The structure algebra $\mathcal{V}_{\mathrm{Ls}}(X)$ lifts from either factor to a Lie algebra of smooth vector fields transversal to $\Delta_{\mathrm{Ls}}$.

We shall label the new boundary hypersurfaces of $X_{s}^{2}$ as $B_{11}\left(X_{s}^{2}\right)$, arising from the first blowup of (4.3), $B_{01}\left(X_{s}^{2}\right)$ arising from the second blowup and $B_{10}\left(X_{s}^{2}\right)$ arising from the third blowup. The lift of the boundary at $\epsilon=0$ will be denoted $B_{00}\left(X_{s}^{2}\right)$; the boundary over $\epsilon=\epsilon_{0}$ will generally 
be ignored. We use the same notation for the boundary hypersurfaces of $\left(X_{s}\right)_{\log }$ and for the boundary hypersurfaces of $X_{\mathrm{Ls}}^{2}$ to which they lift. The remaining boundary hypersurfaces arise from the blow up of both codimension three and codimension two faces in the final step of the definition (4.2). The boundary hypersurfaces arising from codimension three faces will be denoted $B_{22}\left(X_{\mathrm{Ls}}^{2}\right)$, those arising from the codimension two faces will be denoted $B_{21}\left(X_{\mathrm{Ls}}^{2}\right), B_{12}\left(X_{\mathrm{Ls}}^{2}\right), B_{33}\left(X_{\mathrm{Ls}}^{2}\right), B_{20}\left(X_{\mathrm{Ls}}^{2}\right)$ and $B_{02}\left(X_{\mathrm{Ls}}^{2}\right)$ according to whether the face blown up comes from $B_{11}\left(X_{s}^{2}\right) \cap B_{01}\left(X_{s}^{2}\right), B_{11}\left(X_{s}^{2}\right) \cap$ $B_{10}\left(X_{s}^{2}\right), B_{11}\left(X_{s}^{2}\right) \cap B_{00}\left(X_{s}^{2}\right), B_{10}\left(X_{s}^{2}\right) \cap B_{00}\left(X_{s}^{2}\right)$ or $B_{01}\left(X_{s}^{2}\right) \cap B_{00}\left(X_{s}^{2}\right)$. As usual the connectedness properties of these faces depend on the orientation and separation properties of $H$.

We next discuss the structure of these faces. Recall that $B_{0}\left(X_{\mathrm{Ls}}\right)=$ $\bar{M}_{\log }, B_{1}\left(X_{\mathrm{Ls}}\right)=\bar{H}_{\log }$.

Proposition 4.1. There are canonical diffeomorphisms:

$$
\begin{aligned}
& B_{00}\left(X_{\mathrm{Ls}}^{2}\right) \equiv\left(\bar{M}_{\mathrm{tb}}^{2}\right)_{\mathrm{lt}} \equiv\left[\left(B_{0}^{2}\right)_{\mathrm{tb}} ; \tilde{H}^{2} \cap\left\{x^{\prime} / x= \pm 1\right\}\right], \\
& B_{10}\left(X_{\mathrm{Ls}}^{2}\right) \equiv\left(B_{1} \times B_{0}\right)_{\mathrm{tb}}, \\
& B_{01}\left(X_{\mathrm{Ls}}^{2}\right) \equiv\left(B_{0} \times B_{1}\right)_{\mathrm{tb}}, \\
& B_{11}\left(X_{\mathrm{Ls}}^{2}\right) \equiv\left(\bar{H}_{\mathrm{tb}}^{2}\right)_{\mathrm{lt}} \equiv\left[\left(B_{1}^{2}\right)_{\mathrm{tb}} ; \tilde{H}^{2} \cap\left\{x^{\prime} / x= \pm 1\right\}\right], \\
& B_{22}\left(X_{\mathrm{Ls}}^{2}\right) \equiv\left[\left(B_{2}^{2}\right)_{\mathrm{tb}} ; \Delta_{\mathrm{fib}}, \Delta_{\mathrm{fib}}^{-1}\right], \\
& B_{21}\left(X_{\mathrm{Ls}}^{2}\right) \equiv B_{2} \times B_{1}, \\
& B_{12}\left(X_{\mathrm{Ls}}^{2}\right) \equiv B_{1} \times B_{2}, \\
& B_{20}\left(X_{\mathrm{Ls}}^{2}\right) \equiv B_{2} \times B_{0}, \\
& B_{02}\left(X_{\mathrm{Ls}}^{2}\right) \equiv B_{0} \times B_{2}, \\
& B_{33}\left(X_{\mathrm{Ls}}^{2}\right) \equiv\left(B_{2}\right)^{2} .
\end{aligned}
$$

In (4.8) $\Delta_{\mathrm{fib}}\left(\Delta_{\mathrm{fib}}^{-1}\right)$ is the fibre diagonal (anti-diagonal) of $B_{2}^{2}$, for the fibration over $[0,1]$, lifted to $\left(B_{2}^{2}\right)_{\mathrm{tb}}$ and $x, x^{\prime}$ are the original boundary defining functions for $H$ on the left and right factors of $X$ respectively.

Proof. The maps $\pi_{\mathrm{Ls}, L}^{2}$ and $\pi_{\mathrm{Ls}, R}^{2}$ restricted to $B_{m n}$ map to $B_{m}$ and $B_{n}$ respectively, for $(m n) \neq(33)$. In fact $\left(\pi_{\mathrm{Ls}, L}^{2}, \pi_{\mathrm{Ls}, R}^{2}\right)$ lifts from the interior of 


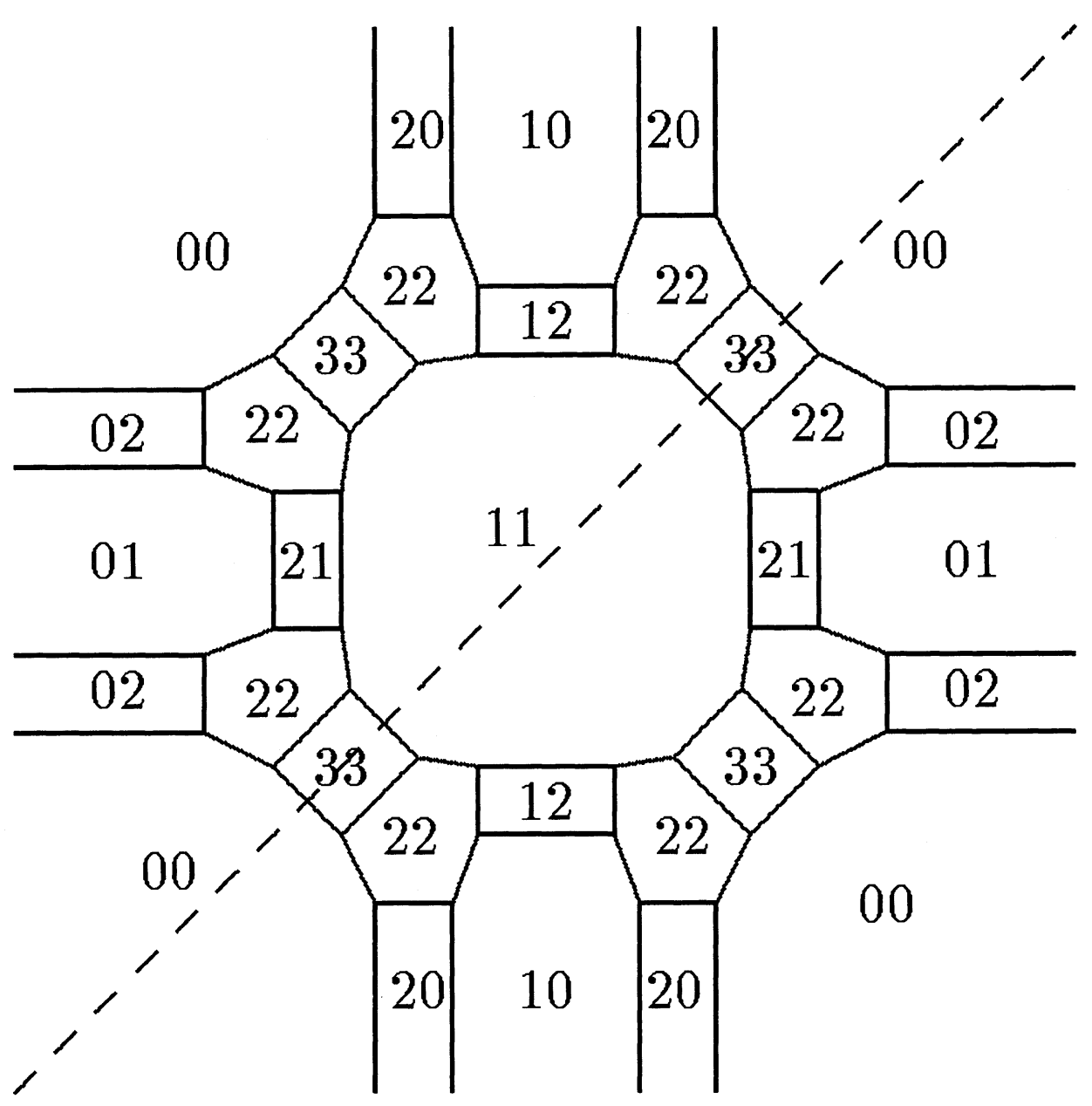

Figure 2. 
$B_{m n}$ to map diffeomorphically to the spaces indicated. The space $B_{33}$ is canonically diffeomorphic to $\widetilde{H}^{2} \times \widetilde{H}^{2} \times[0,1]^{2}$ so (4.13) follows.

\subsection{Densities.}

Lifting the canonical isomorphism

$$
\pi_{L}{ }^{*} \Omega_{b}\left(M \times\left[0, \epsilon_{0}\right]\right) \otimes \pi_{R}{ }^{*} \Omega_{b}\left(M \times\left[0, \epsilon_{0}\right]\right) \equiv \Omega_{b}\left(M^{2} \times\left[0, \epsilon_{0}\right]\right) \otimes \Omega_{b}\left(\left[0, \epsilon_{0}\right]\right)
$$

to the (original) surgery spaces one obtains a canonical isomorphism

$$
\left(\pi_{s, L}^{2}\right)^{*} \Omega_{b}\left(X_{s}\right) \otimes\left(\pi_{s, R}^{2}\right)^{*} \Omega_{b}\left(X_{s}\right) \equiv \Omega_{b}\left(X_{s}^{2}\right) \otimes \Omega_{b}\left(\left[0, \epsilon_{0}\right]\right) .
$$

By Lemma 2.10, the lift of these density bundles to the logarithmic surgery spaces gives the canonical isomorphism

$$
\left(\pi_{\mathrm{Ls}, L}^{2}\right)^{*} \Omega_{D} X_{\mathrm{Ls}} \otimes\left(\pi_{\mathrm{Ls}, R}^{2}\right)^{*} \Omega_{D} X_{\mathrm{Ls}} \equiv \Omega_{D}\left(X_{\mathrm{Ls}}^{2}\right) \otimes \Omega_{c}\left(X_{\mathrm{Ls}}^{0}\right) .
$$

The square root of this density bundle equation,

$$
\left(\pi_{\mathrm{Ls}, L}^{2}\right)^{*} \Omega_{D}^{\frac{1}{2}}\left(X_{\mathrm{Ls}}\right) \otimes\left(\pi_{\mathrm{Ls}, R}^{2}\right)^{*} \Omega_{D}^{\frac{1}{2}}\left(X_{\mathrm{Ls}}\right) \equiv \Omega_{D}^{\frac{1}{2}}\left(X_{\mathrm{Ls}}^{2}\right) \otimes \Omega_{c}^{\frac{1}{2}}\left(X_{\mathrm{Ls}}^{0}\right),
$$

will be used in subsection 4.5 .

\subsection{Logarithmic Surgery Pseudodifferential Operators.}

The purpose of the logarithmic double space is to carry the Schwartz kernels of logarithmic surgery pseudodifferential operators (Ls- $\psi$ dos for short). These will be defined directly in terms of the regularity properties of their kernels. An Ls-pseudodifferential operator acting on half-densities on $M$ (and giving a half-density depending on $\epsilon$ ) will be defined to be a distributional section of $\Omega_{D}^{\frac{1}{2}}\left(X_{\mathrm{Ls}}^{2}\right)$, conormal to $\Delta_{\mathrm{Ls}}$ and with specified behaviour at the boundary of $X_{\mathrm{Ls}}^{2}$. As motivation we first examine a simple example.

Example 2. The kernel of the identity operator acting on multiples of the metric half-density bundle on $M$ is, in the original coordinates $(x, y)$ near $H \subset M$,

$$
\operatorname{Id}=\delta_{x}\left(x^{\prime}\right) \cdot \delta_{y}\left(y^{\prime}\right) \cdot\left|d x \cdot d x^{\prime} \cdot d y \cdot d y^{\prime}\right|^{\frac{1}{2}} .
$$


Let us multiply this by the formal factor $\left|\frac{d(\operatorname{lig} \epsilon)}{(\operatorname{ilg} \epsilon)^{2}}\right|^{\frac{1}{2}}=\left|\frac{d \epsilon}{\epsilon}\right|^{\frac{1}{2}}$ and analyze the resulting half density on $X_{\mathrm{Ls}}^{2}$. Near $B_{00} \cap B_{33}$, using coordinates $x^{\prime} / x$, $\rho_{33}=\operatorname{ilg} x$, and $\rho_{00}=\mathrm{ilg} \epsilon / \rho_{33}$, a short computation gives

$$
\mathrm{Id} \cdot\left|\frac{d(\operatorname{ilg} \epsilon)}{(\operatorname{ilg} \epsilon)^{2}}\right|^{\frac{1}{2}}=\delta\left(\frac{x^{\prime}}{x}-1\right) \cdot \delta_{y}\left(y^{\prime}\right) \cdot\left|\frac{d\left(x^{\prime} / x\right)}{x^{\prime} / x} \cdot \frac{d \rho_{33}}{\rho_{33}^{3}} \cdot \frac{d \rho_{00}}{\rho_{00}^{2}} \cdot d y \cdot d y^{\prime}\right|^{\frac{1}{2}},
$$

and near $B_{33} \cap B_{11}$, with $\rho_{33}=\operatorname{ilg}(\epsilon / x)$ and $\rho_{11}=a \operatorname{ilg} \epsilon / \rho_{33}$,

$$
\mathrm{Id} \cdot\left|\frac{d(\operatorname{ilg} \epsilon)}{(\operatorname{ilg} \epsilon)^{2}}\right|^{\frac{1}{2}}=\delta\left(\frac{x^{\prime}}{x}-1\right) \cdot \delta_{y}\left(y^{\prime}\right) \cdot\left|\frac{d\left(x^{\prime} / x\right)}{x^{\prime} / x} \cdot \frac{d \rho_{33}}{\rho_{33}^{3}} \cdot \frac{d \rho_{11}}{\rho_{11}^{2}} \cdot d y \cdot d y^{\prime}\right|^{\frac{1}{2}} \text {. }
$$

Thus the kernel of the identity is, in these regions and hence everywhere, a distribution supported on and conormal to $\Delta_{\mathrm{Ls}}$; it is clearly elliptic as a $D$-density on $X_{\mathrm{Ls}}^{2}$.

Since $\mathcal{V}_{\mathrm{Ls}}$ lifts from the left factor of $X_{\mathrm{Ls}}^{2}$ to be transversal to $\Delta_{\mathrm{Ls}}$, Lsdifferential operators correspond precisely to $D$-densities supported on the diagonal with arbitrary polynomial symbol.

We define the set of Ls- $\psi$ dos of order $k$ and index family $\mathcal{K}$ (formally) acting from sections of $E$ to sections of $F$ to be the sum of two pieces, the small calculus of order $k$ and the boundary terms of index family $\mathcal{K}$ :

$$
\Psi_{\mathrm{Ls}}^{k, \mathcal{K}}(X ; E, F)=\Psi_{\mathrm{Ls}, \mathrm{sml}}^{k}(X ; E, F)+\Psi_{\mathrm{Ls}, \mathrm{bdy}}^{\mathcal{K}}(X ; E, F) .
$$

When the bundles $E$ and $F$ are equal or trivial (or clear from the context), we simplify the notation to $\Psi_{\mathrm{Ls}}^{k, \mathcal{K}}(X ; E)$ or $\Psi_{\mathrm{Ls}}^{k, \mathcal{K}}(X)$.

The first part of (4.18), the small calculus, is the 'microlocalization' of the space of Ls-differential operators. To take care of the density and bundle factors consider the following bundle (the kernel density bundle) over $X_{\mathrm{Ls}}^{2}$ :

$$
\begin{aligned}
& \mathrm{KD}(E, F)=\beta^{*} \operatorname{Hom}(E, F) \otimes \\
& \otimes\left(\pi_{\mathrm{Ls}, R}^{2}\right)^{*}\left(\Omega_{D}^{\frac{1}{2}}\left(X_{\mathrm{Ls}}\right)\right) \otimes\left(\pi_{\mathrm{Ls}, L}^{2}\right)^{*}\left(\Omega_{D}^{-\frac{1}{2}}\left(X_{\mathrm{Ls}}\right)\right) \otimes \Omega_{D}^{\frac{1}{2}}\left(X_{\mathrm{Ls}}^{2}\right) \\
& \equiv\left(\pi_{\mathrm{Ls}, R}^{2} \otimes \pi_{\mathrm{Ls}, L}^{2}\right)^{*}\left(\operatorname{Hom}\left(E \otimes \Omega_{D}^{-\frac{1}{2}}\left(X_{\mathrm{Ls}}\right)\right), F \otimes \Omega_{D}^{-\frac{1}{2}}\left(X_{\mathrm{Ls}}\right)\right) \otimes \Omega_{D}^{\frac{1}{2}}\left(X_{\mathrm{Ls}}^{2}\right) .
\end{aligned}
$$

Here, on the first line $E$ and $F$ can be either bundles over $M$ or bundles over $X_{s}$ or bundles over $X_{\mathrm{Ls}}$ with $\beta$ interpreted appropriately, in all cases the lift of the homomorphism bundle can be interpreted as a bundle over $X_{\mathrm{Ls}}^{2}$. 
On the second line $E$ and $F$ are bundles over $X_{\mathrm{Ls}}$. In case $E=F=\Omega_{D}^{\frac{1}{2}}$ is isomorphic to the lift to $X_{\mathrm{Ls}}$ of $\Omega_{b}^{\frac{1}{2}}$ from $X_{s}$ the kernel density bundle reduces canonically to $\Omega_{D}^{\frac{1}{2}}\left(X_{\mathrm{Ls}}^{2}\right)$, as is to be expected from (4.17). Then the operators in the small calculus, defined directly as kernels, are

$$
\begin{aligned}
& \Psi_{\mathrm{Ls}, \mathrm{sml}}^{k}(X ; E, F)=\left\{K \in I^{k-\frac{1}{4}}\left(X_{\mathrm{Ls}}^{2} ; \Delta_{\mathrm{Ls}} ; \mathrm{KD}(E, F)\right)\right. \\
& \left.K \text { vanishes rapidly at all boundaries disjoint from } \Delta_{\mathrm{Ls}}\right\} .
\end{aligned}
$$

This notation means that $K$ is 'classical' conormal (1-step polyhomogeneous) to $\Delta_{\mathrm{Ls}}$ of order $k-1 / 4$, with values in the kernel density bundle. The ' $-1 / 4$ ' in the order comes from the the extra $\epsilon$ dimension; this space of kernels corresponds to operators of order $k$ in the usual sense.

The kernels in the boundary part of (4.18) are smooth in the interior of $X_{\mathrm{Ls}}^{2}$ (i.e. have no diagonal singularities) and are polyhomogeneous at all boundaries, with index set $\mathcal{K}\left(B_{\text {gf }}\right)$ at $B_{\text {gf }}$ :

$$
\left.\Psi_{\mathrm{Ls}, \text { bdy }}^{\mathcal{K}}(X ; E, F)=\mathcal{A}_{\mathrm{phg}}^{\mathcal{K}}\left(X_{\mathrm{Ls}}^{2} ; \mathrm{KD}(E, F)\right)\right) .
$$

The fully residual space, $\Psi_{\mathrm{Ls}, \mathrm{bdy}}^{\emptyset}(X)$, is the case where all entries of $\mathcal{K}$ are empty, corresponding to rapid decay at all boundary faces over $\epsilon=0$, in powers of ilg $\epsilon$. Notice that $\Psi_{\mathrm{Ls}, \mathrm{sml}}^{-\infty}(X ; E, F)=\Psi_{\mathrm{Ls}, \mathrm{bdy}}^{\mathcal{K}}(X ; E, F)$ where $\mathcal{K}$ is the index family corresponding to smoothness up to all boundary hypersurfaces meeting $\Delta_{\mathrm{Ls}}$ and rapid decay at all others.

\subsection{Action on Distributions.}

In general the action of $A \in \Psi_{\mathrm{Ls}}^{k, \mathcal{K}}(X ; E, F)$ on $u \in C^{-\infty}\left(X_{\mathrm{Ls}} ; E\right)$ is defined by a generalization of the formula ' $(A u)(x)=\int A(x, y) u(y) d y$ ':

$$
A u \cdot \mu=\left(\pi_{\mathrm{Ls}, L}^{2}\right)_{*}\left(A \cdot\left(\pi_{\mathrm{Ls}, L}^{2}\right)^{*} \mu \cdot\left(\pi_{\mathrm{Ls}, R}^{2}\right)^{*} u \cdot\left|\frac{d(\mathrm{ilg} \epsilon)}{(\mathrm{ilg} \epsilon)^{2}}\right|^{-\frac{1}{2}}\right) .
$$

Here $\mu \in C^{\infty}\left(X_{\mathrm{Ls}} ; \Omega_{D}\right)$ is a non-vanishing section. Equation (4.15) and the definition of the kernel density bundle imply that the argument of $\left(\pi_{\mathrm{Ls}, L}^{2}\right)_{*}$ may be taken to be an $F$-valued (distributional) density. Pushing forward yields an $F$-valued density on $X_{\mathrm{Ls}}$, which when divided by $\mu$ is a distributional section of $F$ independent of the choice of $\mu$. The Pushforward theorem, Theorem 2.3, implies that $\Psi_{\mathrm{Ls}, \mathrm{sml}}^{k}(X ; E, F)$ maps $\mathcal{A}_{\mathrm{phg}}^{\mathcal{F}}\left(X_{\mathrm{Ls}} ; E\right)$ to 
$\mathcal{A}_{\text {phg }}^{\mathcal{F}}\left(X_{\mathrm{Ls}} ; F\right)$ and that $\Psi_{\mathrm{Ls}, \text { bdy }}^{\mathcal{K}}(X ; E, F)$ maps $\mathcal{A}_{\text {phg }}^{\mathcal{F}}\left(X_{\mathrm{Ls}} ; E\right)$ to $\mathcal{A}_{\text {phg }}^{\mathcal{G}}\left(X_{\mathrm{Ls}} ;\right.$ $F$ ) where $\mathcal{G}$ is determined by $\mathcal{K}$ and $\mathcal{F}$. These results are not essential here, so the details are omitted.

\subsection{The Triple Space.}

The purpose of the triple space is to allow the direct analysis of the composite of two Ls- $\psi$ dos. Composition of integral kernels requires three sets of variables, one of which is integrated out. To integrate in the context of Ls- $\psi$ dos we need a 'triple space' with three b-fibrations back to the double space; by the Pushforward theorem the pushforwards under these maps will preserve polyhomogeneity at the boundaries.

The triple logarithmic space is defined by exact analogy with the single and double logarithmic spaces. It is obtained by further blowup from the triple space $X_{s}^{3}$ considered in Part I and [21]:

$$
X_{\mathrm{Ls}}^{3}=\left(X_{s}^{3}\right)_{\mathrm{lt}}=\left(\left(X_{s}^{3}\right)_{\mathrm{log}}\right)_{\mathrm{tb}} .
$$

Another application of Lemma 2.8 shows that there is a commutative diagram:

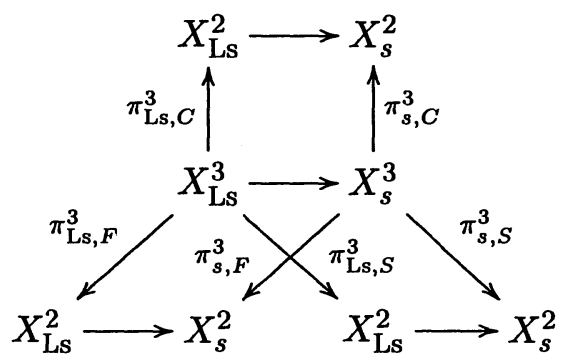

where the maps $\pi_{\mathrm{Ls}, F}^{3}, \pi_{\mathrm{Ls}, C}^{3}$ and $\pi_{\mathrm{Ls}, S}^{3}$ are simple b-fibrations.

The degrees of boundary hypersurfaces of $X_{\mathrm{Ls}}^{3}$ are defined as for the double space, i.e. $d(H)$ is the codimension of the boundary face of $X_{s}^{3}$ from which $H$ arises by blowup. Lemma 2.10 gives

$$
\Omega_{D}\left(X_{\mathrm{Ls}}^{3}\right)=\pi_{\mathrm{lt}}^{*} \Omega_{b} X_{s}^{3}
$$

for the lift of the b-density bundle of $X_{s}^{3}$ to $X_{\mathrm{Ls}}^{3}$, and this in turn gives a canonical isomorphism

$$
\begin{aligned}
\left(\pi_{\mathrm{Ls}, F}^{3}\right)^{*} \Omega_{D}\left(X_{\mathrm{Ls}}^{2}\right) \otimes\left(\pi_{\mathrm{Ls}, C}^{3}\right)^{*} \Omega_{D}\left(X_{\mathrm{Ls}}^{2}\right) \otimes\left(\pi_{\mathrm{Ls}, S}^{3}\right)^{*} \Omega_{D}\left(X_{\mathrm{Ls}}^{2}\right) \equiv & \\
& \equiv\left(\Omega_{D}\left(X_{\mathrm{Ls}}^{3}\right)\right)^{2} \otimes \pi^{*} \Omega_{c} X_{\mathrm{Ls}}^{0}
\end{aligned}
$$


The square root of this density bundle equation

$$
\begin{aligned}
\left(\pi_{\mathrm{Ls}, F}^{3}\right)^{*} \Omega_{D}^{\frac{1}{2}}\left(X_{\mathrm{Ls}}^{2}\right) \otimes\left(\pi_{\mathrm{Ls}, C}^{3}\right)^{*} \Omega_{D}^{\frac{1}{2}}\left(X_{\mathrm{Ls}}^{2}\right) \otimes\left(\pi_{\mathrm{Ls}, S}^{3}\right)^{*} \Omega_{D}^{\frac{1}{2}}\left(X_{\mathrm{Ls}}^{2}\right) \equiv & \\
& \equiv \Omega_{D}\left(X_{\mathrm{Ls}}^{3}\right) \otimes \pi^{*} \Omega_{c}^{\frac{1}{2}} X_{\mathrm{Ls}}^{0}
\end{aligned}
$$

is used below in the composition formula.

\subsection{Composition.}

The composition of two Ls- $\psi$ dos is in general defined by a formula similar to, and arising from, (4.22) for the action on a distribution

$$
A B \cdot \nu=\left(\pi_{\mathrm{Ls}, C}^{3}\right)_{*}\left(\left(\pi_{\mathrm{Ls}, C}^{3}\right)^{*} \nu \cdot\left(\pi_{\mathrm{Ls}, F}^{3}\right)^{*} A \cdot\left(\pi_{\mathrm{Ls}, S}^{3}\right)^{*} B \cdot\left|\frac{d(\mathrm{ilg} \epsilon)}{(\operatorname{ilg} \epsilon)^{2}}\right|^{-\frac{1}{2}}\right) .
$$

Here, $\nu$ is a nonvanishing Ls-half density on $X_{\mathrm{Ls}}^{2}$. Assuming $A \in \Psi_{\mathrm{Ls}}^{k, \mathcal{K}}(X ; E$, $G)$ and $B \in \Psi_{\mathrm{Ls}}^{l, \mathcal{L}}(X ; G, F)$ the terms in the kernel bundle arising from homomorphism bundles compose appropriately, so the expression in the large parentheses is, by (4.25), a section of the bundle

$$
\left(\pi_{\mathrm{Ls}, C}^{3}\right)^{*} \operatorname{Hom}\left(E \otimes \Omega_{D}^{-\frac{1}{2}}\left(X_{\mathrm{Ls}}\right), F \otimes \Omega_{D}^{-\frac{1}{2}}\left(X_{\mathrm{Ls}}\right)\right) \otimes \Omega_{D}^{\frac{1}{2}}\left(X_{\mathrm{Ls}}^{3}\right) .
$$

Therefore it can be pushed forward, at least over the interior, to $X_{\mathrm{Ls}}^{2}$ as $\nu \cdot(A B)$ where $A B$ is a section of the kernel bundle $\mathrm{KD}(E, F)$.

Theorem 4.2. The composition of logarthmic surgery pseudodifferential operators is well defined and

$$
\Psi_{\mathrm{Ls}}^{k, \mathcal{K}}(X ; E, G) \circ \Psi_{\mathrm{Ls}}^{l, \mathcal{L}}(X ; G, F) \subset \Psi_{\mathrm{Ls}}^{k+l, \mathcal{R}}(X ; E, G)
$$

where the dependence of $\mathcal{R}$ on $\mathcal{K}$ and $\mathcal{L}$ is determined by the combinatorial properties of the maps $\pi_{\mathrm{Ls}, O}^{3}$ for $O=F, S, C$; namely for each hypersurface $H^{\prime} \in M_{1}\left(X_{\mathrm{Ls}}^{2}\right)$

$$
\mathcal{R}\left(H^{\prime}\right) \subset
$$

$$
\bigcup_{\left\{H \in M_{1}\left(X_{\mathrm{Ls}}^{3}\right) ; \pi_{\mathrm{Ls}, C}^{3}(H)=H^{\prime}\right\}}\left(\mathcal{K}\left(\pi_{\mathrm{Ls}, S}^{3}(H)\right)+\mathcal{L}\left(\pi_{\mathrm{Ls}, F}^{3}(H)\right)-\left(d(H)-d\left(H^{\prime}\right)\right)\right) .
$$


The 'extended union' in (4.28) is defined by

$$
\begin{aligned}
\mathcal{G} \overline{\mathcal{H}}=\mathcal{G} \cup \mathcal{H} \cup\{(z, p) \mid & \exists\left(z, q_{1}\right) \in \mathcal{G} \\
& \text { and } \left.\left(z, q_{2}\right) \in \mathcal{H} \text { such that } p=q_{1}+q_{2}+1\right\} ;
\end{aligned}
$$

the operation is commutative so extends uniquely to any number of index sets. Notice that the degrees $d(H)$ and $d\left(H^{\prime}\right)$ are actually the codimensions of the boundary faces in $X_{s}^{3}$ and $X_{s}^{2}$ from which the hypersurfaces $H$ and $H^{\prime}$ arise. To prove this result some information on the combinatorial properties of the stretched projections is needed, so we start with

Lemma 4.3. If $H$ disconnects $M$ then any $Q \in M_{1}\left(X_{s}^{2}\right)$ is determined by its images in $M_{1}\left(X_{s}\right)$ under the two stretched projections and any $R \in M_{1}$ $\left(X_{s}^{3}\right)$ is determined by its three images in $M_{1}\left(X_{s}\right)$ under the three stretched projections; furthermore any element of $M^{\prime}\left(X_{s}^{2}\right)$ (respectively $M^{\prime}\left(X_{s}^{3}\right)$ ) is determined by its 'hull' in $M_{1}\left(X_{s}^{2}\right)$ (resp. $M_{1}\left(X_{s}^{3}\right)$ ), this being the set of boundary hypersurfaces which meet a neighbourhood of any one of its interior points.

Proof. The fact that a boundary hypersurface of $X_{s}^{2}$ or $X_{s}^{3}$ is determined by its projections is easily seen from the definitions of the spaces and maps. The assumption that $H$ disconnects $M$ also shows that any intersection of boundary hypersurfaces of one of these spaces is either empty or consists of exactly one boundary face, proving the second part of the lemma.

Proof of Theorem 4.2. The composition formula (4.27) is readily derived from adjoint symmetry and the three special cases

$$
\begin{aligned}
& \Psi_{\mathrm{Ls}, \mathrm{sml}}^{k}(X ; E, G) \circ \Psi_{\mathrm{Ls}, \mathrm{sml}}^{l}(X ; G, F) \subset \Psi_{\mathrm{Ls}, \mathrm{sml}}^{k+l}(X ; E, F), \\
& \Psi_{\mathrm{Ls}, \mathrm{sml}}^{k}(X ; E, G) \circ \Psi_{\mathrm{Ls}, \mathrm{bdy}}^{\mathcal{L}}(X ; G, F) \subset \Psi_{\mathrm{Ls}, \mathrm{bdy}}^{\mathcal{L}}(X ; E, F), \\
& \Psi_{\mathrm{Ls}, \mathrm{bdy}}^{\mathcal{K}}(X ; E, G) \circ \Psi_{\mathrm{Ls}, \mathrm{bdy}}^{\mathcal{L}}(X ; G, F) \subset \Psi_{\mathrm{Ls}, \mathrm{bdy}}^{\mathcal{R}}(X ; E, F),
\end{aligned}
$$

where $\mathcal{R}$ is given by (4.28).

The first of these results shows that the small calculus is closed under composition. Now (4.29) follows easily from the pullback and pushforward 
theorems (with the extension discussed at the end of section 2.3) and the following combinatorial fact concerning the boundary hypersurfaces at which an element of the small calculus can have a non-trivial expansion.

If $Q \in M_{1}\left(X_{\mathrm{Ls}}^{3}\right)$ is such that the interiors of $\pi_{\mathrm{Ls}, F}^{3}(Q)$ and $\pi_{\mathrm{Ls}, S}^{3}(Q)$ both meet $\Delta_{\mathrm{Ls}}$ then $\pi_{\mathrm{Ls}, F}^{3}(Q)=\pi_{\mathrm{Ls}, S}^{3}(Q)=\pi_{\mathrm{Ls}, C}^{3}(Q)$.

Indeed if $\mathcal{J}$ is the index family on $X_{\mathrm{LS}}^{2}$ corresponding to the small calculus then it follows from (4.32) that

$$
\mathcal{J} \supset\left(\pi_{\mathrm{Ls}, C}^{3}\right)_{\#}\left(\left(\pi_{\mathrm{Ls}, F}^{3}\right)^{\#} \mathcal{J} \cdot\left(\pi_{\mathrm{Ls}, S}^{3}\right)^{\#} \mathcal{J}\right)
$$

To see (4.32) recall that $M_{1}\left(X_{\mathrm{Ls}}^{3}\right)=M^{\prime}\left(X_{s}^{3}\right), M_{1}\left(X_{\mathrm{Ls}}^{2}\right)=M^{\prime}\left(X_{s}^{2}\right)$ and $M_{1}\left(X_{\mathrm{Ls}}\right)=M^{\prime}\left(X_{s}\right)$ and that the stretched projections between these spaces induce the same maps (e.g. the map induced by $\pi_{\mathrm{Ls}, F}^{3}$ from $M_{1}\left(X_{\mathrm{Ls}}^{3}\right)$ to $M_{1}\left(X_{\mathrm{Ls}}^{2}\right)$ is the same as that induced by $\pi_{s, F}^{3}$ from $M^{\prime}\left(X_{s}^{3}\right)$ to $\left.M^{\prime}\left(X_{s}^{2}\right)\right)$. An element $S \in M_{1}\left(X_{\mathrm{Ls}}^{2}\right)$ has interior meeting the diagonal if and only if the same is true of the element $B \in M^{\prime}\left(X_{s}^{2}\right)$ from which it arises by blow up. The interior of $B$ meets the diagonal if and only if each element of its hull in $M_{1}\left(X_{s}^{2}\right)$ has the same property. Thus if $Q \in M_{1}\left(X_{\mathrm{Ls}}^{3}\right)$ is to have the stated property that its projections under $\pi_{\mathrm{Ls}, F}^{3}$ and $\pi_{\mathrm{Ls}, S}^{3}$ should both have interiors meeting the diagonal then the element $Q^{\prime} \in M^{\prime}\left(X_{s}^{3}\right)$ from which it arises must have hull in $M_{1}\left(X_{s}^{3}\right)$ each element, $P$, of which has projections under $\pi_{s, F}^{3}$ and $\pi_{s, S}^{3}$ into $M_{1}\left(X_{s}^{2}\right)$ with this same property. It is shown in Part I (and in any case is easy to see) that this implies that the two projections of $P$ into $M_{1}\left(X_{s}\right)$ must be the same. Thus any element of the hull of $Q^{\prime}$ must have all three projections into $M_{1}\left(X_{s}\right)$ the same, hence the three projections of the hull of $Q^{\prime}$ into $M^{\prime}\left(X_{s}\right)$ must be the same. Since a b-fibration is open, the hull of the projection is the projection of the hull, hence all three projections of $Q$ as elements of $M^{\prime}\left(X_{s}\right)$ are the same; this proves (4.32) and hence (4.29).

The second result shows that the boundary terms, for any index family, form an ideal over the small calculus. Again this is a direct consequence of the pullback and pushforward theorems together with a combinatorial fact generalizing (4.32), namely

Given $S \in M_{1}\left(X_{\mathrm{Ls}}^{2}\right)$ there is exactly one $Q \in M_{1}\left(X_{\mathrm{Ls}}^{3}\right)$ such that

$$
\begin{array}{r}
\pi_{\mathrm{Ls}, S}^{3}(Q)=S \text { and } \pi_{\mathrm{Ls}, F}^{3}(Q) \text { has interior meeting } \Delta_{\mathrm{Ls}} \\
\text { and then } \pi_{\mathrm{Ls}, C}^{3}(Q)=S .
\end{array}
$$


This also follows by using an argument with hulls justified by Lemma 4.3. Thus if $Q$ has the stated property then so does an element of the hull, in $M_{1}\left(X_{s}^{3}\right)$ of the element $Q^{\prime} \in M^{\prime}\left(X_{s}^{3}\right)$ from which it arises, i.e. each element of this hull has image under $\pi_{s, F}^{3}$ having its two projections into $M_{1}\left(X_{s}\right)$ equal. It follows that the projections of $Q^{\prime}$ into $M^{\prime}\left(X_{s}^{2}\right)$ under $\pi_{s, S}^{3}$ and $\pi_{s, C}^{3}$ must have the same hulls, and therefore must be equal. Thus the projections of $Q$ into $M_{1}\left(X_{\mathrm{Ls}}^{2}\right)$ under $\pi_{\mathrm{Ls}, S}^{3}$ and $\pi_{\mathrm{Ls}, C}^{3}$ must be equal, which is the content of (4.34).

The third result, that the boundary terms themselves compose with the index families controlled by (4.28) is a direct translation of the pullback and pushforward theorems.

This theorem includes all the composition results listed in section 4.1. Of these, the identification of $\Delta \cdot G$ for $G \in \Psi_{\mathrm{Ls}}^{-2,0}(X ; E)$, is in any case easy to understand, since composition on the left by an Ls-differential operator has an alternative description in terms of lifting Ls-vector fields using $\pi_{\mathrm{Ls}, L}^{2}: X_{\mathrm{Ls}}^{2} \longrightarrow X_{\mathrm{Ls}}$, and letting them act on $G$. It is then immediate that composition with $\Delta_{\epsilon}$ gives a map

$$
\Psi_{\mathrm{Ls}}^{-2, \mathcal{E}}(X ; E) \longrightarrow \Psi_{\mathrm{Ls}}^{0, \mathcal{E}}(X ; E) .
$$

\subsection{An iteratively-residual ideal.}

We shall only explicitly construct the leading terms of the parametrix for the resolvent of $\Delta_{\epsilon}$. The remainder term from this construction will be a harmless 'error term' provided it can be iterated away. Thus we look for an index family $\mathcal{E}$ which defines an iteratively-residual ideal $\mathcal{I}_{R}$ in the sense that

$$
\begin{array}{r}
\mathcal{I}_{R}=\Psi_{\text {Ls,bdy }}^{\mathcal{E}}(X ; E) \text { satisfies } \mathcal{I}_{R}^{k} \subset \Psi_{\text {Ls,bdy }}^{\mathcal{E}_{k}}(X ; E) \\
\text { where } \mathcal{E}_{k} \subset \mathcal{E} \text { and } \mathcal{E}_{k}>r_{k} \rightarrow \infty \text { as } k \rightarrow \infty
\end{array}
$$

Lemma 4.4. There is a natural index family, $\mathcal{E}$, satisfying (4.35) and such that

$$
\left\{\begin{array}{l}
\text { if } d(H)=1, \text { then }(n, 0) \in \mathcal{E}(H) \text { for } n \geq 1 \text { and }(1, k) \in \mathcal{E}(H) \Longrightarrow k=0 \\
\text { if } d(H) \geq 2, \text { then }(n, 0) \in \mathcal{E}(H) \text { for } n \geq 2 \text { and }(2, k) \in \mathcal{E}(H) \Longrightarrow k=0
\end{array}\right.
$$


Proof. Consider the smallest index family for which (4.36) holds, and denote it by $\mathcal{F}$. Thus $\mathcal{F}(H)$ is the index sets $\{(n, 0)\}$ where $n \geq 1$ if $d(H)=1$ and $n \geq 2$ if $d(H)>1$. Let $\mathcal{F}_{2}$ be the index family given by (4.31) for the square of the space $\Psi_{\mathrm{Ls}, \text { bdy }}^{\mathcal{F}}(X ; E)$. Since the b-fibrations involved are all simple this is an integral index family. From (4.31) in order to have $(n, k) \in \mathcal{F}_{2}\left(H^{\prime}\right)$ then must exist elements $\left(n^{\prime}, 0\right) \in \mathcal{F}\left(H_{F}\right)$ and $\left(n^{\prime \prime}, 0\right) \in \mathcal{F}\left(H_{S}\right)$ such that $n \geq n^{\prime}+n^{\prime \prime}-d(H)+d\left(H^{\prime}\right)$ where $H^{\prime}, H_{F}$ and $H_{S}$ are the images of $H \in M_{1}\left(X_{\mathrm{Ls}}^{3}\right)$ under the three stretched projections.

Now for any hypersurfaces $H \in M_{1}\left(X_{\mathrm{Ls}}^{3}\right)$ and $H^{\prime} \in M_{1}\left(X_{\mathrm{Ls}}^{2}\right)$ with $\pi_{\mathrm{Ls}, O}^{3}(H)=H^{\prime}$ there are corresponding boundary faces $h \in M^{\prime}\left(X_{s}^{3}\right)$ and $h^{\prime} \in M^{\prime}\left(X_{s}^{2}\right)$, from which they arise by blowup, which must satisfy $\pi_{s, O}^{3}(h)=h^{\prime}$. Under this map the codimension can decrease by at most 1 . Thus the difference of degrees $d(H)-d\left(H^{\prime}\right)$ is at most 1 . Furthermore, if this difference is 1 then under one of the other two projections the image must have codimension at least 2 . On the other hand if $d\left(H^{\prime}\right) \geq 2$ then all three image of $H$ must have degree at least 2. Combining these combinatorial facts with the definition of $\mathcal{F}$ it follows that $\mathcal{F}_{2}-1$, also satisfies (4.36). In fact this argument shows that if $\mathcal{K}$ and $\mathcal{L}$ are two index families satisfying (4.36) then the index family $\mathcal{R}$ obtained from (4.28) is such that $\mathcal{R}-1$ also satisfies (4.36). Let $\mathcal{F}_{k}$ be the index family arising from $\mathcal{F}$ by $k$-fold composition. Then the minimum order in $\mathcal{F}_{k}$ is $\mathcal{F}+k$, so

$$
\mathcal{E}=\bigcup_{k} \mathcal{F}_{k}
$$

is an index family satisfying the conditions of the lemma.

Thus we can consider an iteratively-residual ideal $\mathcal{I}_{R}$ as in (4.35). For such an ideal it is natural to expect behaviour similar to that of a Volterra operator.

Lemma 4.5. If $R \in \mathcal{I}_{R}$ as in (4.35) then in $\epsilon<\epsilon_{0}$ for some $\epsilon_{0}>0$ depending on $R$

$$
(\mathrm{Id}-R)^{-1}=\operatorname{Id}-S, S \in \mathcal{I}_{R}
$$

Proof. If $R$ is an element of the residual space $\Psi_{\mathrm{Ls}, \mathrm{bdy}}^{\emptyset}(X ; E)$ then $R$ is simply a smoothing operator on $M$ depending smoothly on ilg $\epsilon$ and vanishing rapidly as ilg $\epsilon \rightarrow 0$. It then follows that $(\mathrm{Id}-R)^{-1}=\mathrm{Id}-S$ with $S$ an operator of the same type in $\epsilon<\epsilon_{0}$. If $R \in \mathcal{I}_{R}$ then the formal Neumann series 
for the inverse of $\operatorname{Id}-R$ :

$$
\mathrm{Id}+R+R^{2}+\cdots+R^{k}+\cdots
$$

converges asymptotically, in view of (4.35). Thus the series can be asymptotically summed to an element Id $-S^{\prime}$, with $S^{\prime} \in \mathcal{I}_{R}$ such that $(\operatorname{Id}-R)\left(\operatorname{Id}-S^{\prime}\right)$ $=\mathrm{Id}-R^{\prime}$ with $R^{\prime}$ residual. Thus $\left(\mathrm{Id}-R^{\prime}\right)^{-1}=\mathrm{Id}-S^{\prime \prime}$ with $S^{\prime \prime}$ residual so $S=S^{\prime}+S^{\prime \prime}-S^{\prime} \circ S^{\prime \prime} \in \mathcal{I}_{R}$, proving the lemma.

\subsection{Symbol Map.}

In the ordinary pseudodifferential operator calculus, the symbol map is used as a tool to invert elliptic operators (see [16], Chapter 18). In the logarithmic surgery calculus, we have an analogous symbol map on the small calculus. The symbol of $A \in \Psi_{\mathrm{Ls}, \mathrm{sml}}^{k}(X)$ is the leading part of the Fourier transform of the kernel of $A$ transverse to $\Delta_{\mathrm{Ls}}$; this Fourier transform is, by definition of $\Psi_{\mathrm{Ls}, \mathrm{sml}}^{k}(X)$, a classical symbol. To make the density factors work out, we should divide $A$ by the formal half density factor $\left|\frac{d(\operatorname{lig} \epsilon)}{(\operatorname{ilg} \epsilon)^{2}}\right|^{1 / 2}$, multiply by the half-density $\exp ^{-i\left(\xi \log \left(x^{\prime} / x\right)+\eta \cdot\left(y^{\prime}-y\right)\right)}\left|\frac{d x^{\prime}}{x^{\prime}} d y^{\prime} d \xi d \eta\right|^{1 / 2}$ and integrate over $x^{\prime}$ and $y^{\prime}$, obtaining a half-density. Thus $\sigma_{k}(A)(p, \xi, \eta)\left|\frac{d x}{x} d y d \xi d \eta\right|^{1 / 2}$ is given by

$$
\begin{aligned}
\int e^{-i\left(\xi \log \left(x^{\prime} / x\right)+\eta \cdot\left(y^{\prime}-y\right)\right)} A & \left(p, \frac{x^{\prime}}{x}, y^{\prime}-y\right) \\
\cdot & \psi\left(\left|y-y^{\prime}\right|+\left|\log \frac{x^{\prime}}{x}\right|\right)\left|\frac{d x^{\prime}}{x^{\prime}} d y^{\prime}\right|\left|\frac{d x}{x} d y d \xi d \eta\right|^{\frac{1}{2}} .
\end{aligned}
$$

Here $p \in \Delta_{\mathrm{Ls}}, \psi$ is a cutoff function and we use $\log \left(x^{\prime} / x\right), y^{\prime}-y$ for coordinates transverse to $\Delta_{\mathrm{Ls}}$. The half-density written here is a canonical factor on $N^{*} \Delta_{\mathrm{Ls}}$, so can be cancelled; the symbol then is a function on $N^{*} \Delta_{\mathrm{Ls}}$, which is polyhomogeneous on the fibres. As is to be expected it is the same as the lift of the symbol defined in Part I on $\Delta_{s}$, lifted to $\Delta_{\text {Ls }}$. The principal symbol $\sigma_{k}(A)$ is homogeneous of degree $k$ and is independent of choice of coordinates. There is a quantization map from symbols of order $k$ to $\Psi_{\mathrm{Ls}, \mathrm{sml}}^{k}(X)$ given by inverse Fourier transform of the symbol in the $(\xi, \eta)$ directions. Thus the symbol map may be used, as in the usual case, to solve operator equations $P \cdot G=\mathrm{Id}, P \in \Psi_{\mathrm{Ls}, \mathrm{sml}}^{k}(X)$ elliptic, up to errors in $\Psi_{\mathrm{Ls}, \mathrm{sml}}^{-\infty}(X)$. However, such errors are not uniformly compact as $\epsilon \downarrow 0$, since 
elements of $\Psi_{\mathrm{Ls}, \mathrm{sml}}^{-\infty}(X)$ do not vanish at the boundaries $B_{00}, B_{11}$ or $B_{33}$. To construct parametrices for elliptic operators one therefore also needs to solve model problems at these boundary faces.

Lemma 4.6. $\Delta_{\epsilon}$ is an elliptic $L s-\psi d o$.

Proof. Away from $B_{1}$ and $B_{2}$ this is true because the metric is nondegenerate. At $B_{1}$ and $B_{2}$ we have, up to a symbol of at most second order vanishing to infinite order (in Taylor series) at $H$ :

$$
\sigma_{2}(\Delta)(\xi, \eta)=|\xi|^{2}+|\eta|^{2} .
$$

\subsection{Model Operators.}

In this subsection we define model operators for $A \in \Psi_{\mathrm{Ls}}^{k, 0}(X)$. The model operator $N_{m n}$ at face $B_{m n}$ is in principle just the restriction of $A$ to $B_{m n}$. The restrictions are defined as at the end of section 2.1, that is, by division by the canonical factor $\left|d \rho_{m n} /\left(\rho_{m n}(\mathrm{ilg} \epsilon)^{d}\right)\right|^{1 / 2}$; this maps into the degree half density bundle as described in section 2.1.

These model operators have different characters. The first of them, $N_{00}$, maps into a blown up version of the cusp pseudodifferential calculus, $\Psi_{c}^{k, 0}\left(\bar{M}_{\log }\right)$. This set of operators (Schwartz kernels) is, by definition, the space of $D$-half densities on the cusp double space

$$
\left.\bar{M}_{\log }^{2} ; \tilde{H}^{2} ; \tilde{H}^{2} \cap\left\{x^{\prime} / x=1\right\}\right]
$$

conormal to the diagonal. Equation (4.4) shows that $B_{00}$ is canonically this space with the boundary of the 'anti-diagonals' also blown up. It also shows that it is a blowup of the b-stretched product $M_{b}^{2}$ of [22]. Under this blowup, b-pseudodifferential operators lift to cusp pseudodifferential operators; all the models considered in this paper will be lifts of b-pseudodifferential operators. The model $N_{11}$ behaves analogously for $\bar{H}$.

The model operator $N_{33}$ maps $\Psi_{\mathrm{Ls}, \mathrm{sml}}^{k}(X)$ to a family of operators in $\Psi_{c \text { sml }}^{k}(\bar{H})$ parameterized smoothly by $s$, the coordinate in (3.5) along $\Delta_{\mathrm{Ls}} \cap$ $B_{33}$. This is because the lifts of $\mathcal{V}_{\mathrm{Ls}}$ from both the left and right are tangent to the leaves $s=$ constant $\left(=s^{\prime}\right)$. The operator on each leaf acts by convolution on $\bar{H}$. For an operator $B$ lifted from $\Psi_{\mathrm{Ls}, \mathrm{sml}}^{k}(X), N_{33}(B)$ will be the indicial 
operator of $B$ (in the terminology of [22] and Part I) on each leaf on which $s$ takes a constant value, but in general the $N_{33}(A)$ will depend on $s$.

The other models $N_{01}, N_{10}, N_{12}, N_{21}, N_{02}, N_{20}$ and $N_{22}$ map $\Psi_{\mathrm{Ls}}^{k, 0}(X)$ to the space $C^{\infty}\left(N_{m n} ; \Omega_{D}^{1 / 2} B_{m n}\right)$, since these faces are disjoint from the diagonal.

To construct the resolvent of $\Delta$ we will need to know the model operators of $\Delta \cdot G$.

Lemma 4.7. In the notation of Proposition 3.4 we have

$$
\begin{aligned}
N_{m n}(\Delta \cdot G) & =R_{m}(\Delta) \cdot N_{m n}(G),(m n) \neq(33) \\
N_{33}(\Delta \cdot G) & =R_{1}(\Delta) \cdot N_{33}(G) .
\end{aligned}
$$

Proof. Lemma 4.1 identifies the various boundary hypersurfaces $B_{m n} \neq B_{33}$ as blown up products of boundary hypersurfaces of $X_{\mathrm{Ls}}$. Since $\Delta$ acts on $G$ by products of $\mathcal{V}_{\mathrm{Ls}}$-vector fields lifted from the left, which are tangent to the boundary, (4.40) follows. On $B_{33}$, using coordinates $s, \tilde{r}=\log \left(x^{\prime} / x\right), y, y^{\prime}$, the vector field $V_{0}$ lifts from the left to be $\frac{\partial}{\partial \tilde{r}}$, so (4.41) follows.

Finally, we discuss the reduced normal operator $\operatorname{RN}(\Delta)$. The following is a double space version of Lemma 3.7.

Lemma 4.8. Suppose $N_{2 n}(\Delta G)=0$, that is, $\Delta_{H}\left(N_{2 n}(G)\right)=0$. Let $W$ be a normal vector field to $B_{2 n}$ tangent to the leaves $\{s, y=$ constant $\}$ and tangent to the right stretched projection to $X_{\mathrm{Ls}}$. If $\Delta_{H}\left(W(G)\left\lceil B_{2 n}\right)=0\right.$ then $\Delta G$ vanishes to second order at $B_{2 n}$ and

$$
N_{2 n}\left(\frac{\Delta G}{(\operatorname{ias} \epsilon)^{2}}\right)=D_{s}^{2}\left(N_{2 n}(G)\right)
$$

The result follows from Lemma 3.7.

\section{One-dimensional surgery resolvent.}

We return to the reduced normal operator of section 3.4. This model operator does not appear in Part I and is precisely the new ingredient which allows us to construct the surgery parametrix down to $\lambda=0$. 


\subsection{Scaling property.}

Let $\bar{K}\left(s, s^{\prime}, z\right)\left|d s \cdot d s^{\prime}\right|^{1 / 2}$ be the Schwartz kernel of $D_{s}^{2}-z^{2}$ on the interval $[-1,1]_{s}$, acting on functions $\bar{u}$ taking values in the vector space $V=\operatorname{Ker}\left(\Delta_{H}\right)$, with boundary conditions as in (3.28). To illustrate the scaling property of this operator, let $K \cdot\left|d g_{\epsilon} d g_{\epsilon}^{\prime}\right|^{1 / 2}$ be the kernel of $\left(\Delta_{\epsilon}-(\text { ias } \epsilon)^{2} z^{2}\right)^{-1}$ on $V$-valued functions defined on $[-1,1]_{x}$ with surgery metric $g_{\epsilon}=d x^{2} /\left(x^{2}+\epsilon^{2}\right)$ and with boundary conditions as in Proposition 3.8. Arclength is

$$
r=\int_{0}^{x} \frac{d \tilde{x}}{\sqrt{\tilde{x}^{2}+\epsilon^{2}}}=\sinh ^{-1} \frac{x}{\epsilon}
$$

so the length of the interval with respect to $g_{\epsilon}$ is $2 \sinh ^{-1}(1 / \epsilon) \equiv 2 L_{\epsilon}$.

Consider the function ias $\epsilon \equiv 1 / \sinh ^{-1}(1 / \epsilon)=L_{\epsilon}^{-1}$. Then

$$
\operatorname{ias} \epsilon=\operatorname{ilg} 2 \epsilon+O\left((\operatorname{ilg} \epsilon)^{\infty}\right)=\operatorname{ilg} \epsilon(1+(\log 2) \operatorname{ilg} \epsilon)^{-1}+0\left((\operatorname{ilg} \epsilon)^{\infty}\right)
$$

is a $C^{\infty}$ function of ilg $\epsilon$, equal to it to first order; however as the discussion above shows, ias $\epsilon$ is a more natural function to use than ilg $\epsilon$ in this setting. Let $s$ be the rescaled arclength, $s=r / L_{\epsilon}$, so $s \in[-1,1]$ for all $\epsilon$. Then $\Delta_{\epsilon}=D_{r}^{2}=(\text { ias } \epsilon)^{2} D_{s}^{2}$, so

$$
\begin{aligned}
(\operatorname{ias} \epsilon)^{2}\left(D_{s}^{2}-z^{2}\right) K & =\delta\left(r-r^{\prime}\right) \\
& =(\operatorname{ias} \epsilon) \delta\left(s-s^{\prime}\right) .
\end{aligned}
$$

Hence, using coordinates $s, s^{\prime}, K$ scales in $\epsilon$ as (ias $\left.\epsilon\right)^{-1}$ :

$$
K\left(s, s^{\prime}, \epsilon, z\right)=(\operatorname{ias} \epsilon)^{-1} \bar{K}\left(s, s^{\prime}, z\right) .
$$

Let us now multiply $K$ by the canonical half density factor

$$
\nu=\left|d g_{\epsilon} d g_{\epsilon}^{\prime} d(\mathrm{ilg} \epsilon) /(\mathrm{ilg} \epsilon)^{2}\right|^{1 / 2}
$$

and lift to the logarithmic double space $X_{\mathrm{Ls}}^{2}\left([-1,1]_{x}\right)$.

Lemma 5.1. The lift of $K \cdot \nu$ to $X_{\mathrm{Ls}}^{2} \times \mathbb{C}_{z}$ is a $D$-half-density meromorphic in $z$, conormal to $\Delta_{\mathrm{Ls}}$ and (ias $\left.\epsilon\right)^{-1} \times$ smooth up to all boundary hypersurfaces; that is, $K$ is a meromorphic family of $L s-\psi d$ dos with index family -1 . The restriction of (ias $\epsilon) K \cdot \nu$ to $B_{22}$ is the resolvent $\left(D_{s}^{2}-z^{2}\right)^{-1}$. 
Proof. All these assertions follow easily from (5.1). Meromorphy in $z$ follows from meromorphy of the resolvent $\bar{K}=\left(\Delta-z^{2}\right)^{-1}$. To show that it lifts to be conormal to $\Delta_{\mathrm{Ls}}$, observe that $\bar{K}\left(s, s^{\prime}, z\right)=-\left(\frac{1}{2}\right)\left|s-s^{\prime}\right|+K^{\prime}$ with $K^{\prime}$ a smooth function of $\left(s, s^{\prime}, z\right)$. Since $s$ is a smooth function on $X_{\mathrm{Ls}}$, (ias $\epsilon)^{-1} K^{\prime}\left(s, s^{\prime}, z\right)$ lifts to be (ias $\left.\epsilon\right)^{-1} \times$ smooth on $X_{\mathrm{Ls}}^{2}$. We need only show that (ias $\epsilon)^{-1}\left(\frac{1}{2}\right)\left|s-s^{\prime}\right|$ lifts to be conormal on $X_{\mathrm{Ls}}^{2}$. This is clear away from $\epsilon=0$, so we need only check this in Taylor series at $\epsilon=0$. Near the interior of $B_{11}$, (ias $\left.\epsilon\right)^{-1}\left|s-s^{\prime}\right|=\left|\sinh ^{-1}(x / \epsilon)-\sinh ^{-1}\left(x^{\prime} / \epsilon\right)\right|$. Since $x / \epsilon, x^{\prime} / \epsilon$ are coordinates on the interior of $B_{11}$, this is conormal to $\Delta_{\mathrm{Ls}}=\left\{x / \epsilon=x^{\prime} / \epsilon\right\}$. Near $B_{33}$ and $B_{00}$,

$$
(\operatorname{ias} \epsilon)^{-1}\left(s-s^{\prime}\right)=\left(\log (x / \epsilon)-\log 2-\log \left(x^{\prime} / \epsilon\right)+\log 2+O\left(\rho_{33}^{\infty} \rho_{00}^{\infty}\right)\right)
$$

so (ias $\epsilon)^{-1}\left|s-s^{\prime}\right|=\left|\log \left(x / x^{\prime}\right)\right|+O\left(\rho_{33}^{\infty} \rho_{00}^{\infty}\right)$ which is conormal to $\Delta_{\mathrm{Ls}}=$ $\left\{\log \left(x / x^{\prime}\right)=0\right\}$. The last assertion follows immediately from (5.1).

\subsection{Scattering Matrix.}

Let us calculate the scattering matrix, as defined in [22], Chapter 6, and in section 6.2 below, for the two one-dimensional Laplacians $N_{0}(\Delta)=\Delta_{0}$ on $M_{ \pm}=[-1,0]_{x}$ and $[0,1]_{x}$. On $[-1,0]$ and $[0,1] \Delta_{0}=-\left(x D_{x}\right)^{2}$ is a bLaplacian near $x=0$, and looks like $-D_{x}^{2}$ at $x= \pm 1$, with mixed boundary conditions as above. The scattering solutions of $\left(\Delta_{0}-z^{2}\right) u=0$ are defined by analytic continuation from $\operatorname{Im} z>0$ by

$$
\left(\Delta-z^{2}\right) S(\phi, z)=0 \text {, with } S(\phi, z)=x^{i z} \phi+\psi \text { near } x=0, \phi \in V, \psi \in L^{2} .
$$

To satisfy the boundary conditions, $S(\phi, z)$ must be a linear combination of

$$
\left\{\left(x^{i z}+x^{-i z}\right) v,\left(x^{i z}-x^{-i z}\right) w ; v \in \Lambda_{ \pm}^{D}, w \in \Lambda_{ \pm}^{N}\right\} .
$$

The scattering matrix $S_{ \pm}(z)$ is the linear transformation that maps the coefficient of $x^{-i z}$ to the coefficient of $x^{i z}$; thus $S_{ \pm}(z)(v-w)=v+w$ and hence $S_{ \pm}(z) v=v$ for $v \in \Lambda_{ \pm}^{D}$ and $S_{ \pm}(z) w=-w$ for $w \in \Lambda_{ \pm}^{N}$. Hence

$$
S_{ \pm}(z)=\operatorname{proj} \Lambda_{ \pm}^{D}-\operatorname{proj} \Lambda_{ \pm}^{N},
$$

independently of $z$. We also see from this that the smooth and logarithmically growing null spaces of $\Delta_{0}$ are precisely $\Lambda_{ \pm}^{D}$ and $\Lambda_{ \pm}^{N}$. It follows that the reduced normal operator of $\Delta_{\epsilon}-(\text { ias } \epsilon)^{2} z^{2}$ on $[-1,1]_{x}$ with boundary conditions as above is the operator $D_{s}^{2}-z^{2}$ with the same boundary conditions. 
In this model case, the reduced normal operator 'reproduces' the original operator.

\subsection{Properties at the boundary.}

The boundary conditions associated to $\Delta$ imply that

$$
\begin{aligned}
\bar{K}\left( \pm 1, s^{\prime}, z\right) & \in \operatorname{Hom}\left(V, \Lambda_{ \pm}^{D}\right), \\
\partial_{s} \bar{K}\left( \pm 1, s^{\prime}, z\right) & \in \operatorname{Hom}\left(V, \Lambda_{ \pm}^{N}\right) .
\end{aligned}
$$

Because $\Delta$ is self-adjoint, we also have

$$
\begin{aligned}
\bar{K}(s, \pm 1, z) & \in \operatorname{Hom}\left(\Lambda_{ \pm}^{D}, V\right), \\
\partial_{s^{\prime}} \bar{K}(s, \pm 1, z) & \in \operatorname{Hom}\left(\Lambda_{ \pm}^{N}, V\right) .
\end{aligned}
$$

Therefore, $\bar{K}( \pm 1, \pm 1, z) \in \operatorname{Hom}\left(\Lambda_{ \pm}^{D}, \Lambda_{ \pm}^{D}\right)$ and $\bar{K}( \pm 1, \mp 1, z) \in \operatorname{Hom}\left(\Lambda_{ \pm}^{D}, \Lambda_{\mp}^{D}\right)$. Also, since $\left(\Delta-z^{2}\right) \cdot \bar{K}=\delta\left(s-s^{\prime}\right)$,

$$
\lim _{s^{\prime} \uparrow s} \partial_{s} \bar{K}\left(s, s^{\prime}, z\right)=\operatorname{Id}+\lim _{s^{\prime} \downarrow s} \partial_{s} \bar{K}\left(s, s^{\prime}, z\right) .
$$

Hence

$$
\begin{aligned}
\operatorname{Hom}\left(\Lambda_{+}^{N}, V\right) \ni \lim _{s \rightarrow 1} \partial_{s^{\prime}} \bar{K}(s, 1, z) & =\lim _{s^{\prime} \rightarrow 1}\left(\partial_{s} \bar{K}\left(1, s^{\prime}, \bar{z}\right)\right)^{*} \\
& =-\operatorname{Id}+\lim _{s \rightarrow 1}\left(\partial_{s} \bar{K}(s, 1, \bar{z})\right)^{*}
\end{aligned}
$$

and $\lim _{s^{\prime} \rightarrow 1}\left(\partial_{s} \bar{K}(s, 1, \bar{z})\right)^{*} \in \operatorname{Hom}\left(V, \Lambda_{+}^{N}\right)$. It follows that

$$
\begin{aligned}
\partial_{s^{\prime}} \bar{K}(1-0,1, z) & =-\operatorname{proj} \Lambda_{+}^{N}+A(z) \\
\partial_{s} \bar{K}(1-0,1, z) & =\operatorname{proj} \Lambda_{+}^{D}+A^{*}(\bar{z}) \\
\partial_{s^{\prime}} \bar{K}(1,1-0, z) & =\operatorname{proj} \Lambda_{+}^{D}+A(z) \\
\partial_{s} \bar{K}(1,1-0, z) & =-\operatorname{proj} \Lambda_{+}^{N}+A^{*}(\bar{z}) .
\end{aligned}
$$

$$
\text { where } A(z) \in \operatorname{Hom}\left(\Lambda_{+}^{N}, \Lambda_{+}^{D}\right)
$$

Similar results hold at $s=s^{\prime}=-1$.

\subsection{Eigenvalues.}

The spectrum of $\mathrm{RN}(\Delta)$ is, by standard elliptic theory, a discrete sequence $0 \leq z_{0}^{2}<z_{1}^{2}<\cdots$ with each $z_{j}^{2}$ of finite multiplicity. The kernel 
$\bar{K}\left(s, s^{\prime}, z\right)$ of $\left(\mathrm{RN}(\Delta)-z^{2}\right)^{-1}$ is meromorphic in $z$ with poles only at the $\pm z_{j}$. These poles are simple for all $z_{j} \neq 0$ with residue equal to $\left(2 z_{j}\right)^{-1}$ times the projection onto the $j$ th eigenspace and has a double pole at 0 if $z_{0}=0$, with residue zero and coefficient of $z^{-2}$ equal to the projection onto the null space. This different behaviour at 0 is just the result of using $z^{2}$ rather than $z$ as the spectral parameter.

We next discuss the eigenvalues of the reduced normal Dirac operator, since these contribute to our formula for the limit of the eta invariant. Recall from section 3 that the reduced normal operator of $\partial_{\epsilon}$ at $B_{2}$ is $\gamma D_{s}$ acting on $\operatorname{Ker}\left(\partial_{H}\right)$-valued functions $\bar{u}$ on the interval $[-1,1]$, where $\gamma \equiv \operatorname{cl}\left(d x / \sqrt{x^{2}+\epsilon^{2}}\right)$ is the matrix $\left(\begin{array}{cc}1 & 0 \\ 0 & -1\end{array}\right)$ with respect to the splitting of the spinor bundle $S=S^{+} \oplus S^{-}$at $B_{2}$. This model operator has boundary conditions $\bar{u} \uparrow \pm 1 \in \Lambda_{\tilde{\gamma}, \pm}$, where the $\Lambda_{\tilde{\delta}, \pm}$ are the spaces of $C^{\infty}$ solutions $\phi$ to $\widetilde{\partial}_{M_{ \pm}} \phi=0$. Of course, $\Lambda_{\widetilde{\sigma}, \pm}$ are the same as $\Lambda_{ \pm}^{D}$.

Notice that if $\bar{u}$ is an eigenfunction of $\gamma D_{s}$ with eigenvalue $z$ then $\bar{u}=$ $A e^{i z s}+B e^{-i z s}$, where $\gamma A=A$ and $\gamma B=-B$. This satisfies the boundary conditions only if $A+B e^{2 i z} \in \Lambda_{-}^{D}$ and $A+B e^{-2 i z} \in \Lambda_{+}^{D}$. But notice that $A e^{i\left(z+k \frac{\pi}{2}\right) s}+(-1)^{k} B e^{-i\left(z+k \frac{\pi}{2}\right) s}$ also satisfies the equation and these boundary conditions, and so is an eigenfunction of $\gamma D_{s}$ with eigenvalue $z+\frac{k \pi}{2}$. Hence the eigenvalues of $\gamma D_{s}$ are periodic with period $\frac{\pi}{2}$. By Weyl's law, there must be precisely $\operatorname{dim} \operatorname{Ker}\left(\partial_{H}\right)$ eigenvalues in the interval $[0, \pi)$.

\subsection{Heat kernel and large $|z|$ asymptotics of $\bar{K}$.}

One can write down an explicit formula for the heat kernel $e^{-t \mathrm{RN}(\Delta)}$ using the reflection principle. This is a convenient way to obtain the large $|z|$ asymptotics of $\bar{K}\left(s, s^{\prime}, z\right)$ and to compute the eta invariant of $\mathrm{RN}(\widetilde{\partial})$.

When reflecting $\phi \in V$ at $s= \pm 1$, one should take $+\phi$ if $\phi \in \Lambda_{ \pm}^{D}$ and $-\phi$ if $\phi \in \Lambda_{ \pm}^{N}$; that is, in general one should take $S_{ \pm} \phi$, where $S_{ \pm}=$ $\operatorname{proj} \Lambda_{ \pm}^{D}-\operatorname{proj} \Lambda_{ \pm}^{N}$ is the scattering matrix for $\Delta_{M_{ \pm}}$at zero energy. There are four 'series' of reflections, originating from $\phi$ at $s$ ' : they are

$$
\begin{gathered}
\left(S_{+} S_{-}\right)^{k} S_{+} \phi \text { at } 4 k+2-s^{\prime}, \\
\left(S_{-} S_{+}\right)^{k} S_{-} \phi \text { at }-4 k-2-s^{\prime}, \\
\left(S_{+} S_{-}\right)^{k} \phi \text { at } 4 k+s^{\prime} \text { and } \\
\left(S_{-} S_{+}\right)^{k} \phi \text { at }-4 k+s^{\prime} .
\end{gathered}
$$


Thus $e^{-t \mathrm{RN}(\Delta)}\left(s, s^{\prime}\right)$ is given by

$$
\begin{array}{r}
\frac{1}{\sqrt{4 \pi t}}\left\{e^{-\left|s-s^{\prime}\right|^{2} / 4 t} \mathrm{Id}+\sum_{k=0}^{\infty}\left[e^{-\left|4 k+s^{\prime}-s\right|^{2} / 4 t}\left(S_{+} S_{-}\right)^{k}+\right.\right. \\
+e^{-\left|-4 k+s^{\prime}-s\right|^{2} / 4 t}\left(S_{-} S_{+}\right)^{k}+e^{-\left|4 k+2-s-s^{\prime}\right|^{2} / 4 t}\left(S_{+} S_{-}\right)^{k} S_{+} \\
\left.\left.+e^{-\left|-4 k-2-s-s^{\prime}\right|^{2} / 4 t}\left(S_{-} S_{+}\right)^{k} S_{-}\right]\right\} .
\end{array}
$$

From this one can use the transform

$$
\left(\Delta-z^{2}\right)^{-1}=\int_{0}^{\infty} e^{-t \Delta} e^{t z^{2}} d t
$$

to obtain an infinite sum for the model resolvent $\bar{K}$ from which large $|z|$ asymptotics $(\operatorname{Im} z<0)$ are easy to determine. Choosing a contour of integration for (5.8) so that $\operatorname{Re} t>0$ and $\operatorname{Re} t z^{2}<0$ consider the transform applied to a term

$$
\frac{1}{\sqrt{4 \pi t}} e^{-\left|A \pm s-s^{\prime}\right|^{2} / 4 t} \cdot B
$$

of (5.7). If $\left|A \pm s-s^{\prime}\right| \neq 0$ then

$$
\int_{0}^{\infty} \frac{1}{\sqrt{4 \pi t}} e^{-\left|A \pm s-s^{\prime}\right|^{2} / 4 t} e^{t z^{2}} d t=\frac{1}{2 i z} e^{-i z\left|A \pm s-s^{\prime}\right|}
$$

is rapidly decreasing as $|z| \rightarrow \infty$, uniformly in any sector $-\pi+\delta<\arg z<$ $-\delta$. Hence, for $\left(s, s^{\prime}\right)$ away from $(1,1)$ and $(-1,-1)$ the only term that contributes to (polynomial) asymptotics in $z$ is the first, $(4 \pi t)^{-1 / 2} e^{-\left|s-s^{\prime}\right|^{2} / 4 t} \cdot$ Id and for $\left(s, s^{\prime}\right)$ near $( \pm 1, \pm 1)$, the only terms that contribute are

$$
\frac{1}{\sqrt{4 \pi t}}\left(e^{-\left|s-s^{\prime}\right|^{2} / 4 t} \operatorname{Id}+e^{-\left| \pm 2-s-s^{\prime}\right|^{2} / 4 t} S_{ \pm}\right) \text {. }
$$

Thus, performing the integral (5.8), we get, uniformly in any sector as above and for $\left(s, s^{\prime}\right)$ in a closed set disjoint from $(-1,-1)$ and $(1,1)$,

$$
\bar{K}\left(s, s^{\prime}, z\right)=\frac{e^{-i z\left|s-s^{\prime}\right|}}{2 i z} \operatorname{Id}+O\left(e^{-C|z|}\right)
$$

and for $\left(s, s^{\prime}\right)$ near the corners $( \pm 1, \pm 1)$,

$$
\begin{array}{r}
\bar{K}\left(s, s^{\prime}, z\right)=\frac{1}{2 i z}\left(e^{-i z\left|s-s^{\prime}\right|}+e^{-i z\left| \pm 2-s-s^{\prime}\right|}\right) \operatorname{proj} \Lambda_{ \pm}^{D} \\
+\frac{1}{2 i z}\left(e^{-i z\left|s-s^{\prime}\right|}-e^{-i z\left| \pm 2-s-s^{\prime}\right|}\right) \operatorname{proj} \Lambda_{ \pm}^{N}+O\left(e^{-C|z|}\right)
\end{array}
$$


Comparing these formulae with (5.2), we see that $A(z)$ is exponentially decreasing as $|z| \rightarrow \infty$ in this sector.

\subsection{Eta invariant.}

We now explicitly evaluate the eta invariant of $\mathrm{RN}(\widetilde{\partial})$ in terms of the subspaces $\Lambda_{ \pm}^{D}, \Lambda_{ \pm}^{N}$ which determine $\mathrm{RN}(ð)$. To state this result, decompose the vector space $V$ into an orthogonal direct sum $V=V_{1} \oplus V_{2}$, where

$$
\begin{aligned}
& V_{1}=\Lambda_{+}^{D} \cap \Lambda_{-}^{D} \oplus \Lambda_{+}^{N} \cap \Lambda_{-}^{N} \oplus \Lambda_{+}^{D} \cap \Lambda_{-}^{N} \oplus \Lambda_{-}^{D} \cap \Lambda_{+}^{N} \\
& V_{2}=V \ominus V_{1} .
\end{aligned}
$$

$V_{1}$ is a 'trivial' subspace, a sum of vector spaces in which the boundary conditions are either Dirichlet or Neumann at each end. With respect to this direct sum, $\gamma$ and the scattering matrices $S_{ \pm}=\operatorname{proj} \Lambda_{ \pm}^{D}-\operatorname{proj} \Lambda_{ \pm}^{N}$ split; write $\gamma^{r}, S_{ \pm}^{r}$ for $\gamma \backslash V_{2}, S_{ \pm}\left\lceil V_{2}\right.$. The Dirac operator RN(ð) therefore splits

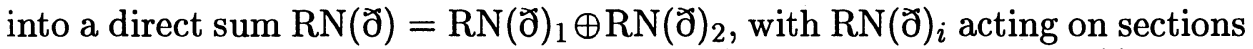
of $V_{i}$. Define the 'superdeterminant' Sdet of an operator $A=\left(\begin{array}{cc}A^{(1)} & 0 \\ 0 & A^{(-1)}\end{array}\right)$ on $V_{2}$, diagonal with respect to $\gamma^{r}$, by

$$
\operatorname{Sdet} A=\operatorname{det} A^{(1)}\left(\operatorname{det} A^{(-1)}\right)^{-1} \text {, assuming } \operatorname{det} A^{(-1)} \neq 0 .
$$

Proposition 5.2. The eta invariant of $\mathrm{RN}(\widetilde{\partial})$ is given by

$$
\eta(\mathrm{RN}(ð))=\frac{i}{\pi} \log \operatorname{Sdet}\left(\operatorname{Id}-S_{+}^{r} S_{-}^{r}\right) .
$$

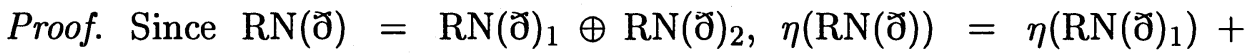
$\eta\left(\mathrm{RN}(\widetilde{\partial})_{2}\right)$. But $\mathrm{RN}(ð)_{1}$ is spectrally symmetric, so $\eta\left(\mathrm{RN}(\widetilde{\delta})_{1}\right)=0$. Hence we just need to calculate $\eta\left(\mathrm{RN}(\widetilde{\partial})_{2}\right)$. To simplify notation, assume that $V_{1}=\{0\}$, so that $\operatorname{RN}(\widetilde{\delta})_{2}$ is just $\mathrm{RN}(\widetilde{\partial})$.

Recall that the eigenvalues of $\mathrm{RN}(\widetilde{\partial})$ are periodic with period $\pi / 2$. It is well known that the eta invariant of an operator with eigenvalues $k \pi / 2+\alpha$ is $1-\frac{4 \alpha}{\pi}$ for $\alpha \neq 0$. Observe that $\operatorname{dim} \Lambda_{ \pm}^{D}=\operatorname{dim} \Lambda_{ \pm}^{N}=\frac{1}{2} \operatorname{dim} V$. For if not, then the sum of the dimensions of some two of these subspaces would be bigger than $\operatorname{dim} V$ and then they would have to intersect nontrivially. Thus $R N(\widetilde{\partial})$ has no zero eigenvalues, because the multiplicity of 0 as an eigenvalue is precisely $\operatorname{dim} \Lambda_{+}^{D} \cap \Lambda_{-}^{D}$. Therefore the eta invariant is the sum

$$
\sum_{j} 2\left(1-\frac{4 \alpha_{j}}{\pi}\right)
$$


over eigenvalues $\alpha_{j} \in\left(0, \frac{\pi}{2}\right)$.

If $\alpha \in(0, \pi / 2)$ is an eigenvalue of $\operatorname{RN}(\delta)$, then we can write the corresponding eigenfunction as

$$
e^{i \alpha s} \phi+e^{-i \alpha s} \psi
$$

where $\gamma \phi=\phi$ and $\gamma \psi=-\psi$. The boundary conditions imply that

$$
\begin{aligned}
\phi+e^{-2 i \alpha} \psi & \in \Lambda_{+}^{D} \\
\phi+e^{2 i \alpha} \psi & \in \Lambda_{-}^{D} .
\end{aligned}
$$

Projecting off $\Lambda_{ \pm}^{D}$ and applying $\gamma$ yields the two sets of equations

$$
\begin{aligned}
& -\operatorname{proj}_{\Lambda_{+}^{N}} \phi=-\operatorname{proj}_{\Lambda_{+}^{N}} e^{-2 i \alpha} \psi \quad \operatorname{proj}_{\Lambda_{+}^{D}} \phi=\operatorname{proj}_{\Lambda_{+}^{D}} e^{-2 i \alpha} \psi \\
& \operatorname{proj}_{\Lambda_{-}^{N}} \phi=-\operatorname{proj}_{\Lambda_{-}^{N}} e^{2 i \alpha} \psi \quad \operatorname{proj}_{\Lambda_{-}^{D}} \phi=\operatorname{proj}_{\Lambda_{-}^{D}} e^{2 i \alpha} \psi
\end{aligned}
$$

whence

$$
\begin{array}{ll}
S_{+} \phi=e^{-2 i \alpha} \psi & S_{+} \psi=e^{2 i \alpha} \phi \\
S_{-} \phi=e^{2 i \alpha} \psi & S_{-} \psi=e^{-2 i \alpha} \phi
\end{array}
$$

and so finally

$$
\left(I-S_{+} S_{-}\right) \phi=\left(1-e^{4 i \alpha}\right) \phi, \quad\left(I-S_{+} S_{-}\right) \psi=\left(1-e^{-4 i \alpha}\right) \psi .
$$

By Weyl's Law and the $\pi / 2$-periodicity of eigenvalues of $\mathrm{RN}(ð)$, it follows that $\alpha \in(0, \pi / 2)$ is an eigenvalue of $\mathrm{RN}(\widetilde{\partial})$ if and only if $e^{ \pm 4 i \alpha}$ are eigenvalues of $S_{+} S_{-}$on the \pm 1 eigenspaces of $\gamma$.

Finally,

$$
\begin{aligned}
& \log \operatorname{Sdet}\left(\operatorname{Id}-S_{+} S_{-}\right)=\log \prod_{j} \frac{1-e^{4 i \alpha_{j}}}{1-e^{-4 i \alpha_{j}}} \\
&=\log \prod_{j} e^{i\left(4 \alpha_{j}-\pi\right)}=\sum_{j} i\left(4 \alpha_{j}-\pi\right) .
\end{aligned}
$$

Here we are using the standard branch of the logarithm. Multiplying by $\frac{i}{\pi}$ yields (5.11), so (5.2) is established.

This calculation has also been done by Lesch and Wojciechowski [19]. Their formula, which they denote $m\left(\Lambda_{+}, \Lambda_{-}\right)$, has a somewhat different form than ours, but it is easy to check that the two agree. Bunke [5] relates 
this term to the Maslov index: it may also be calculated by averaging the Maslov index $\tau\left(\Lambda_{+}, \Lambda_{-}, g \Lambda\right)$ as $g$ ranges over the group of symplectomorphisms fixing $\gamma$, where $\Lambda$ is any fixed Lagrangian in $V$.

\section{Resolvent with scaled spectral parameter.}

In section 4.1 a brief outline of the construction of the resolvent of $\Delta$ was given. In this section we carry out the details of this construction, and prove Theorem 1.1. Here, as in the one-dimensional example, we write the spectral parameter $\lambda=($ ias $\epsilon) z$ to capture the behaviour of small eigenvalues, and treat $z$ as a parameter. In the next section we will construct the 'full' resolvent on a bigger space with contains both the region $X_{\mathrm{Ls}}^{2} \times \mathbb{C}_{z}$ and $X_{\mathrm{Ls}}^{2} \times(\mathbb{C} \backslash \mathbb{R})_{\lambda}$ and unifies the resolvent constructed in this section with that constructed in Part I.

As the construction is rather lengthy, we begin by outlining the strategy in more detail.

\subsection{Strategy of the Construction.}

The first step in the construction is to build a parametrix $G(z)$ for $\left(\Delta_{\epsilon}-\right.$ $\left.(\text { ias } \epsilon)^{2} z^{2}\right)^{-1}$; this means that we need to solve the operator equation

$$
\left(\Delta_{\epsilon}-(\operatorname{ias} \epsilon)^{2} z^{2}\right) G(z)=\mathrm{Id}+R(z),
$$

with the 'error term' $R(z)$ in the iteratively-residual ideal of Lemma 4.4. Initially (in subsections $6.2-6.7$ ) we shall work under two simplifying restrictions: that $z^{2}$ lies away from the discrete spectrum of $\mathrm{RN}(\Delta)$ and that $\Delta_{0}=\Delta_{\bar{M}}$ has no $L^{2}$ null space. Under those restrictions we will construct a holomorphic family $G(z)$ in the space $\Psi_{\mathrm{Ls}}^{-2,-1}(X)$ satisfying (6.1); in fact, it will be a two-sided parametrix, with $G(z)\left(\Delta_{\epsilon}-(\text { ias } \epsilon)^{2} z^{2}\right)$ - Id also in the iteratively-residual ideal. The index family -1 is motivated by Lemma 5.1. Equation (6.1) involves satisfying three types of conditions: the full symbol equations across $\Delta_{\mathrm{Ls}}$, as always in the pseudodifferential approach to elliptic equations; model equations at each boundary hypersurface at $\epsilon=0$, as described in section 4 and compatibility conditions for models at intersections of adjacent boundary hypersurfaces. The compatibility conditions are those for compatibility of Taylor series at a corner. In section 6.2 we list the model equations that need to be solved, write down the compatibility condition between models at adjacent faces, and summarize results about b-Laplacians from [22]. Then in the following three sections we define models, order by 
order, and check compatibility conditions.

The key to solving for the model operators is to identify the top term (growing as (ias $\epsilon)^{-1}$ ) at $B_{22}$. This is shown in subsection 6.3 to be uniquely determined by the model equations and compatibility conditions, and is given, exactly as in Lemma 5.1 , by the resolvent $\left(\operatorname{RN}(\Delta)-z^{2}\right)^{-1}$. Once this term is identified, the solutions to the other models are defined.

In sections 6.6 and 6.7 we perform steps (ii) and (iii) of section 4.1, obtaining the resolvent $\left(\Delta_{\epsilon}-(\operatorname{ias} \epsilon)^{2} z^{2}\right)^{-1}$ subject to the two restrictions noted above. In the following two subsections we remove these restrictions, first extending the construction to $z$ near $\operatorname{spec} \operatorname{RN}(\Delta)$ and then treating the case that $\Delta_{0}$ does have $L^{2}$ null space. Finally we show that the 'very small eigenvalues', corresponding to $z=0$, are actually exponentially decreasing in (ias $\epsilon)$, i.e. vanish as a power of $\epsilon$.

\subsection{Preliminaries.}

Model Operator Equations at each face To construct the parametrix it is necessary to construct several terms in the Taylor series at each boundary hypersurface of $X_{\mathrm{Ls}}^{2}$. To specify this precisely we need to discuss coordinate choices near each hypersurface. Fix coordinates $(x, y)$ near $H$ corresponding to a product decomposition of a neighbourhood of $H \subset M$. Let $p$ denote any coordinate system on $\bar{M}_{\log }$ and define $r=\sinh ^{-1}(x / \epsilon), s=($ ias $\epsilon) r$, $\xi=\operatorname{ilg} x$ as before. Denote the metric density on $H$ by $d h$ and the surgery metric density $d g_{\epsilon}\left|\frac{d(\operatorname{ilg} \epsilon)}{(\operatorname{ilg} \epsilon)^{2}}\right|$ on $X_{\mathrm{Ls}}$ by $\mu$. On $X_{\mathrm{Ls}}$, denote the set of functions $r, y$ by $\mathcal{C}_{1}, s, y$ by $\mathcal{C}_{2}$, and $p$ by $\mathcal{C}_{0}$. Then the functions in $\mathcal{C}_{i}$ are coordinates on the interior of $B_{i}$. Denote

$$
\begin{aligned}
& \mu_{1}=d r \cdot d h \\
& \mu_{2}=d s \cdot d h \\
& \mu_{0}=d g_{0}
\end{aligned}
$$

Lemma 6.1. The density $\mu$ can be written

$$
\mu=\mu_{i}\left|\frac{d(\operatorname{ilg} \epsilon)}{(\operatorname{ilg} \epsilon)^{2}}\right|(\operatorname{ias} \epsilon)^{d\left(B_{i}\right)-1}+O\left(\rho_{i}^{\infty}\right) .
$$

On $X_{\mathrm{Ls}}^{2}$ recall that every face $B_{m n} \neq B_{33}$ is canonically isomorphic to a blown up version of $B_{m} \times B_{n}$. Using primes to denote coordinates lifted from the right stretched projection $X_{\mathrm{Ls}}^{2} \rightarrow X_{\mathrm{Ls}}$, denote the collection of functions $\mathcal{C}_{m} \cup \mathcal{C}_{n}^{\prime}$ by $\mathcal{C}_{m n}$, write $\mathcal{C}_{33}=\left\{s, \log \left(x^{\prime} / x\right), y, y^{\prime}\right\}$, and write $\nu$ 
for the canonical density $d g_{\epsilon} \cdot d g_{\epsilon}^{\prime}\left|\frac{d(\mathrm{ig} \epsilon)}{(\operatorname{lig} \epsilon)^{2}}\right|$. Then the functions in $\mathcal{C}_{m n}$ are coordinates in the interior of $B_{m n}$. Define $\nu_{m n}=\mu_{m} \otimes \mu_{n}^{\prime}$, and $\nu_{33}=$ $d s\left(\log \left(x^{\prime} / x\right)\right)^{-2} d \log \left(x^{\prime} / x\right) d h d h^{\prime} ;$ then $\nu_{m n}$ is a smooth $D$-density on $B_{m n}$ and, corresponding to the above lemma, at each boundary hypersurface

$$
\nu=\nu_{m n}\left|\frac{d(\mathrm{ilg} \epsilon)}{(\mathrm{ilg} \epsilon)^{2}}\right|(\operatorname{ias} \epsilon)^{d\left(B_{m n}\right)-1}+O\left(\rho_{m n}^{\infty}\right)
$$

near $B_{m n}$. We shall use these coordinates and density extension results to extend models, defined initially on one boundary hypersurface $B_{m n}$, to a neighbourhood of $X_{\mathrm{Ls}}^{2}$.

The models of $G$ are $D$-half densities on $B_{m n}$; the top order models we denote

$$
G_{m n}^{(-1)}=N_{m n}((\operatorname{ias} \epsilon) G)
$$

since $G$ is postulated to have growth of order -1 at each boundary hypersurface. Here $N_{m n}$ is the restriction, or normal operator, defined in section 4.10. If $A_{m n}$ is a $D$-half-density on $B_{m n}$, then write

$$
A_{m n}=a\left(\mathcal{C}_{m n}\right) \cdot \nu_{m n}^{\frac{1}{2}}
$$

where this notation means that the function $a$ is written in terms of the functions in $\mathcal{C}_{m n}$. Then

$$
\tilde{A}_{m n}=a\left(\mathcal{C}_{m n}\right) \cdot \nu^{\frac{1}{2}}
$$

is an extension to a smooth $D$-half density in a neighbourhood of $B_{m n}$. We then define inductively

$$
G_{m n}^{(j)}=N_{m n}\left((\operatorname{ias} \epsilon)^{-j}\left[G-\sum_{k=-1}^{j-1}(\operatorname{ias} \epsilon)^{k} \tilde{G}_{m n}^{(k)}\right]\right) .
$$

We emphasize that the higher order models depend on the choice of coordinates $\mathcal{C}_{m n}$. At each boundary hypersurface $G$ has a 'Taylor series' in ias $\epsilon$, using the coordinates $\mathcal{C}_{m n}$ in the interior of each boundary hypersurface:

$$
\begin{aligned}
& G=\sum_{j=-1}^{0}(\operatorname{ias} \epsilon)^{j} G_{m n}^{(j)}+O(\operatorname{ias} \epsilon) \text { near } B_{m n}, d\left(B_{m n}\right)=1 \\
& G=\sum_{j=-1}^{1}(\operatorname{ias} \epsilon)^{j} G_{m n}^{(j)}+O\left((\operatorname{ias} \epsilon)^{2}\right) \text { near } B_{m n}, d\left(B_{m n}\right) \geq 2 .
\end{aligned}
$$


This is not a true Taylor series, since (ias $\epsilon$ ) is a valid boundary defining function only in the interior of each face; thus, for $G \in \Psi_{\mathrm{Ls}}^{-2,-1}(X)$, the model $G_{m n}^{(j)}$ may have growth of order $\rho_{p q}^{-j-1}$ at an adjacent boundary hypersurface $B_{p q}$. These are the only models we need to consider, as the higher order models are already error terms in (6.1).

Lemmas 3.5, 4.7 and 4.8 shows how the Laplacian acts on models $B_{m n} \neq$ $B_{33}$. For $B_{33}$ in terms of coordinates $y, y^{\prime}, \log \left(x^{\prime} / x\right), s$ the Laplacian looks like

$$
\Delta=I(\Delta)+v \cdot Q=-\left(\nabla_{\log \left(x^{\prime} / x\right)}\right)^{2}+\Delta_{H}+v \cdot Q
$$

where $v$ vanishes to infinite order at $B_{33}$ and $Q$ is a Ls- $\psi$ do of order at most two. The model equations involving the identity operator are

$$
\begin{aligned}
\Delta_{\bar{M}} G_{00}^{(0)} & =G_{00}^{(0)} \Delta_{\bar{M}}=\operatorname{Id}_{\bar{M}} \\
\Delta_{\bar{H}} G_{11}^{(0)} & =G_{11}^{(0)} \Delta_{\bar{H}}=\operatorname{Id}_{\bar{H}} \\
\Delta_{\bar{H}} G_{33}^{(0)} & =G_{33}^{(0)} \Delta_{\bar{H}}=\operatorname{Id}_{\bar{H}} .
\end{aligned}
$$

The other equations for $j=-1$ or 0 are

$$
R_{m}(\Delta) G_{m n}^{(j)}=G_{m n}^{(j)} R_{n}(\Delta)=0
$$

for all $m n$ when $j=-1$, and for $m n \neq 00,11,33$ when $j=0$. The equations for $j=1$ are a little more complicated. They are

$$
\begin{aligned}
\Delta_{\bar{M}} G_{02}^{(1)} & =z^{2} G_{02}^{(-1)} \\
\Delta_{\bar{H}} G_{12}^{(1)} & =z^{2} G_{12}^{(-1)} \\
\Delta_{\bar{H}} G_{33}^{(1)} & =z^{2} G_{33}^{(-1)} \\
G_{20}^{(1)} \Delta_{\bar{M}} & =z^{2} G_{20}^{(-1)} ; \\
G_{21}^{(1)} \Delta_{\bar{H}} & =z^{2} G_{21}^{(-1)} \\
\Delta_{H} G_{2 m}^{(1)} & =-\left(D_{s}^{2}-z^{2}\right) G_{2 m}^{(-1)} \\
G_{m 2}^{(1)} \Delta_{H} & =-\left(D_{s^{\prime}}^{2}-z^{2}\right) G_{m 2}^{(-1)} .
\end{aligned}
$$

The last two equations contain even more information. As $\Delta_{H} G_{2 m}^{(1)}$ is orthogonal to $\operatorname{Ker} \Delta_{H}$ and $\left(D_{s}^{2}-z^{2}\right) G_{2 m}^{(-1)}$ is in $\operatorname{Ker} \Delta_{H}$, both must be zero. A similar statement holds for $G_{m 2}^{(1)}$. So we get

$$
\begin{array}{r}
\Delta_{H} G_{2 m}^{(1)}=G_{m 2}^{(1)} \Delta_{H}=0 \\
\left(D_{s}^{2}-z^{2}\right) G_{2 m}^{(-1)}=\left(D_{s^{\prime}}^{2}-z^{2}\right) G_{m 2}^{(-1)}=0 .
\end{array}
$$


Note that the reduced normal operator only appears at order $j=-1$ because it acts two terms down in the Taylor series.

Let us make a simplifying observation about the models $G_{22}^{(0)}$ and $G_{22}^{(1)}$. The only requirement for these models is that they be smooth and $\operatorname{Ker}\left(\Delta_{H}\right) \otimes \operatorname{Ker}\left(\Delta_{H}\right)$-valued. It is possible to define such a model, compatible with all adjacent faces, if and only if the adjacent faces are $\operatorname{Ker}\left(\Delta_{H}\right) \otimes \operatorname{Ker}\left(\Delta_{H}\right)$-valued and compatible between themselves at the intersection with $B_{22}$. This condition is always satisfied below, so we will ignore these models from now on.

Compatibility between models on adjacent faces If the model operators are continuous functions, it is sufficient to check compatibility between models on adjacent faces in the interior of the intersection. Suppose $B_{m n}$ and $B_{p q}$ intersect, and suppose that $\rho_{m n}$ and $\rho_{p q}$ are boundary defining functions, valid in the interior of $B_{m n} \cap B_{p q}$, such that $\rho_{m n} \rho_{p q}=$ ias $\epsilon$. On each face $G$ has a 'Taylor series'

$$
\sum_{i \geq-1}(\operatorname{ias} \epsilon)^{i} G_{m n}^{(i)} \text { or } \sum_{j \geq-1}(\operatorname{ias} \epsilon)^{j} G_{p q}^{(j)}
$$

and at $B_{m n} \cap B_{p q}, G_{m n}^{(i)}$ (resp. $\left.G_{p q}^{(j)}\right)$ has a Taylor series in $\rho_{p q}$ (resp. $\rho_{m n}$ ), which we write

$$
\sum_{j \geq-1} \rho_{p q}^{j-i}\left(G_{m n}^{(i)}\right)_{p q, j-i} \quad\left(\text { resp. } \sum_{i \geq-1} \rho_{m n}^{i-j}\left(G_{p q}^{(j)}\right)_{m n, i-j}\right)
$$

Comparing, we see

$$
\left(G_{m n}^{(i)}\right)_{p q, j-i}=\left(G_{p q}^{(j)}\right)_{m n, i-j}
$$

is a necessary and sufficient condition for these models to be compatible.

It is useful to observe here that the sections $\Phi^{(j)}$ defined below have expansions of the form

$$
\Phi^{(j)}=\sum_{k=0}^{j}(\log x)^{k} b_{k}(y)+O\left(x^{\delta}\right)
$$

at $x=0$; they have no terms in their Taylor series in ilg $x$ vanishing faster than a constant. Many of the $\left(G_{p q}^{(j)}\right)_{m n, i-j}$ will therefore turn out to be zero, for $i>j$. This simplifies the checking of compatibility.

Resolvent of a b-Laplacian Let us start by considering the model equation (6.3):

$$
\Delta_{\bar{M}} G_{00}^{(0)}=G_{00}^{(0)} \Delta_{\bar{M}}=\operatorname{Id}_{\bar{M}} .
$$


Let us recall some facts from [22] regarding the resolvent $\left(\Delta_{\bar{M}}-\lambda^{2}\right)^{-1}$. In Chapter 6 of [22], it is shown that $\Delta_{\bar{M}}-\lambda^{2}$ is invertible in the bpseudodifferential calculus for $\operatorname{Im} \lambda<0$ with the inverse extending meromorphically to a neighbourhood of 0 in $\mathbb{C}_{\lambda}$. Provided $\Delta_{\bar{M}}$ has no $L^{2}$-null space, there is only a simple pole at $\lambda=0$, and the residue there is the projection onto the bounded null space of $\Delta_{\bar{M}}$. Thus at $\lambda=0,\left(\Delta_{\bar{M}}-\lambda^{2}\right)^{-1}$ has a Laurent series

$$
\left(\Delta_{\bar{M}}-\lambda^{2}\right)^{-1}=\sum_{j \geq-1} \lambda^{j} \operatorname{Res} \frac{(j)}{M}
$$

with $\operatorname{Res} \frac{(-1)}{M}=-i \operatorname{proj} C^{\infty} \operatorname{Ker}$. Applying $\left(\Delta-\lambda^{2}\right)$ to (6.9) shows that $\Delta_{\bar{M}} \cdot \operatorname{Res} \frac{(0)}{M}=\mathrm{Id}$, so that $\operatorname{Res} \frac{(0)}{M}$ is an inverse to $\Delta_{\bar{M}}$ (though not of course a bounded operator on $L_{b}^{2}$ ), and for $j \geq 1, \Delta_{\bar{M}} \operatorname{Res} \frac{(j)}{M}=\operatorname{Res} \frac{(j-2)}{M}$. If $\Delta_{\bar{M}}$ does have $L^{2}$ null space, then the series (6.9) must be replaced with one starting at $\lambda=-2$, with $\operatorname{Res} \frac{(-2)}{M}=-\operatorname{proj}_{L^{2}} \operatorname{Ker}$. Then we have $\Delta_{\bar{M}} \cdot \operatorname{Res} \frac{(0)}{M}=$ Id $-\operatorname{proj}_{L^{2}}$ Ker . Similar results hold for $\Delta_{\bar{H}}$; but, since $\bar{H}$ has a product metric, $\Delta_{\bar{H}}$ never has $L^{2}$ null space.

We shall also need the notion of scattering solutions and scattering matrix. For definiteness we will describe these just for $M_{+}$. Let $\left\{\phi_{J}\right\}$ be an orthonormal basis for $V$, which splits into $\left\{\phi_{i}\right\}, 1 \leq i \leq \operatorname{dim} \Lambda_{+}^{D}$, a basis for $\Lambda_{+}^{D}$, and $\left\{\phi_{\alpha}\right\}, \operatorname{dim} \Lambda_{+}^{D}+1 \leq \alpha \leq \operatorname{dim} V$, a basis for $\Lambda_{+}^{N}$. We will use, in the summation convention, the form of the index - capital, small roman or small greek - to determine whether the sum is over a basis of $V, \Lambda_{+}^{D}$ or $\Lambda_{+}^{N}$. The scattering solutions $\Phi_{J}(\lambda)$, with $\lambda$ near $0, \operatorname{Im} \lambda<0$, are the solutions to

$$
\left(\Delta-\lambda^{2}\right) \Phi_{J}(\lambda)=0
$$

with the boundary behaviour $\Phi_{J}(\lambda) \sim x^{-i \lambda} \phi_{J}(y)+v$, with $v \in L_{b}^{2}$ near $H$. They have an expansion

$$
2 \Phi_{J}(\lambda)=x^{-i \lambda} \phi_{J}(y)+x^{i \lambda} S_{J K}(\lambda) \phi_{K}(y)+O\left(x^{\delta}\right),
$$

with $\delta>0$ uniformly near $\lambda=0$. Here $S$ is the scattering matrix; it is meromorphic near $\lambda=0$, symmetric, unitary for $\lambda$ real, and satisfies $S(\lambda) S(-\lambda)=$ Id . From this this it follows that $S(0)=\left(\begin{array}{cc}\text { Id } & 0 \\ 0-\text { Id }\end{array}\right)$ and $\partial_{\lambda} S(0)$ is block diagonal with respect to the splitting $V=\Lambda_{+}^{D} \oplus \Lambda_{+}^{N}$.

Let us denote $(1 / k !)\left(\frac{\partial}{\partial \lambda}\right)^{k} \Phi_{J}(\lambda)_{\lceil\lambda=0}$ by $\Phi_{J}^{(k)}$, and $(1 / k !)\left(\frac{\partial}{\partial \lambda}\right)^{k} S_{J K}(\lambda)_{\lceil\lambda=0}$ by $S_{J K}^{(k)}$. By differentiating (6.10) with respect to $\lambda$, we obtain

$$
\Delta_{\bar{M}} \Phi_{J}^{(0)}=\Delta_{\bar{M}} \Phi_{J}^{(1)}=0 \text { and } \Delta_{\bar{M}} \Phi_{J}^{(2)}=\Phi_{J}^{(0)} .
$$


We conclude that $\Phi_{\alpha}^{(0)} \equiv 0, \Phi_{j}^{(1)}=S_{j k}^{(1)} \Phi_{k}^{(0)}$, and $\Phi_{j}^{(0)}$ (respectively $i \Phi_{\alpha}^{(1)}$ ) form the bounded (resp. logarithmically growing) null space of $\Delta_{\bar{M}}$.

We need to compute the top terms in the (polyhomogeneous) expansion of $\left(\Delta_{\bar{M}}-\lambda^{2}\right)^{-1}$ at the faces of $B_{00}$, that is, those terms with exponent tending to zero as $\lambda \rightarrow 0$. At the 'front face', $x=x^{\prime}=0$, corresponding to $B_{00} \cap B_{33}$, we have

$$
\begin{aligned}
\left(\Delta_{\bar{M}}-\lambda^{2}\right)^{-1} \sim \frac{1}{2 i \lambda}\left(\left(x^{\prime} / x\right)^{ \pm i \lambda} \phi_{J}(y) \phi_{J}\left(y^{\prime}\right)+\right. & \\
& \left.+x^{i \lambda} x^{i \lambda} \phi_{J}(y) S_{J K}(\lambda) \phi_{K}\left(y^{\prime}\right)\right), \frac{x^{\prime}}{x} \lessgtr 1
\end{aligned}
$$

At the left boundary $x / x^{\prime}=0$, corresponding to $B_{00} \cap B_{20}$, we have

$$
\left(\Delta_{\bar{M}}-\lambda^{2}\right)^{-1} \sim \frac{1}{2 i \lambda}\left(x^{i \lambda} \phi_{J}(y) \Phi_{J}\left(p^{\prime}\right)\right)
$$

and at the right boundary $x^{\prime} / x=0$, corresponding to $B_{00} \cap B_{02}$, we have

$$
\left(\Delta_{\bar{M}}-\lambda^{2}\right)^{-1} \sim \frac{1}{2 i \lambda}\left(x^{\prime i \lambda} \Phi_{J}(p) \phi_{J}\left(y^{\prime}\right)\right) .
$$

The 'T' notation In defining models and checking compatibility we will exploit as much as possible the models $K_{m n}^{(j)}$ of the reduced normal operator studied in section 5 . The space $\operatorname{Ker} \Delta_{H}$ has been viewed in two different ways: as a subspace of smooth sections over $H$, and as an abstract vector space $V$. In the latter guise it has played the role of a trivial vector bundle over $[-1,1]$, sections of which are acted on by the reduced normal operator. If $\phi \in V$ then denote the section over $H$ to which it corresponds by $\mathfrak{T}(\phi)$, the 'transfer' of $\phi$. We transfer continuous sections on $B_{1}$ and $B_{2}$ from the logarithmic single space corresponding to $[-1,1]$ to $X_{\mathrm{Ls}}(M)$ according to

$$
\begin{aligned}
& \mathfrak{T}\left(a_{I}(r) \phi_{I}\right)=a_{I}(r) \mathfrak{T}\left(\phi_{I}\right)(y) \text { on } B_{1} ; \\
& \mathfrak{T}\left(b_{I}(s) \phi_{I}\right)=b_{I}(s) \mathfrak{T}\left(\phi_{I}\right)(y) \text { on } B_{2} .
\end{aligned}
$$

On $B_{+0}\left(X_{\mathrm{Ls}}([-1,1])\right)$ the sections $\Phi_{J}^{(k)}$ are given by

$$
\begin{aligned}
\Phi_{j}^{(k)} & =\left\{\begin{array}{l}
0 \text { if } k \text { is odd } ; \\
1 / k ! \phi_{j}(\log x)^{k} \text { otherwise }
\end{array}\right. \\
\Phi_{\alpha}^{(k)} & =\left\{\begin{array}{l}
0 \text { if } k \text { is even; } \\
1 / k ! \phi_{\alpha}(\log x)^{k} \text { otherwise. }
\end{array}\right.
\end{aligned}
$$


Define the transfer on the $\mathbb{C}$-span of these sections by

$$
\mathfrak{T}\left(\Phi_{J}^{(k)}\right)=\left\{\begin{array}{l}
0 \text { if } \Phi_{J}^{(k)}=0 \\
\Phi_{J}^{(k)}\left(M_{+}\right) \text {otherwise. }
\end{array}\right.
$$

Thus $\mathfrak{T}\left(\Phi_{J}^{(k)}\right)$ has the same leading behaviour as $\Phi_{J}^{(k)}$ at the boundary but may have lower order terms depending on derivatives of $S_{J_{K}}$ at $\lambda=0$.

We also want the transfer defined on sections over boundary hypersurfaces of $X_{\mathrm{Ls}}^{2}$. Recall that $B_{m n} \neq B_{33}$ is a blowup of $B_{m} \times B_{n}$. Let $\pi_{L}, \pi_{R}$ be the stretched projections to $B_{m}$ and $B_{n}$. We define the transfer on sections of $B_{m n}$ which are sums of products of sections on $B_{m}$ and $B_{n}$ on which T is defined above. It is defined by

$$
\mathfrak{T}\left(\sum \pi_{L}^{*} \psi_{L} \otimes \pi_{R}^{*} \psi_{R}\right)=\sum \pi_{L}^{*} \mathfrak{T}\left(\psi_{L}\right) \otimes \pi_{R}^{*} \mathfrak{T}\left(\psi_{R}\right)
$$

On $B_{33}$, define

$$
\mathfrak{T}\left(a_{I J}\left(s, \log \left(x^{\prime} / x\right)\right) \phi_{I} \otimes \phi_{J}^{\prime}\right)=a_{I J}\left(s, \log \left(x^{\prime} / x\right)\right) \mathfrak{T}\left(\phi_{I}\right)(y) \mathfrak{T}\left(\phi_{J}\right)\left(y^{\prime}\right) .
$$

The transfer is not defined on sections which are not of the above form. It will be applied to certain models of the reduced normal operator in the construction of models for our parametrix $G$.

\subsection{Terms of order $(\operatorname{ias} \epsilon)^{-1}$.}

Consider equations (6.6) and (6.7) for $j=-1$. One might at first guess that these models should be zero, because there are no nonzero terms on the right hand side of these equations. Surprisingly, perhaps, this is not the case. Recall that in the one dimensional case, $K_{22}^{(-1)}$ is actually the resolvent of the model problem $\left(\mathrm{RN}(\Delta)-z^{2}\right)^{-1}$ (See Lemma 5.1). The general case behaves in the same way:

Proposition 6.2. The model equations (6.6), (6.7) for $G_{22}^{(-1)}$ and the compatibility conditions (6.8) have the unique solution

$$
G_{22}^{(-1)}=\mathfrak{T}\left(K_{22}^{(-1)}\right)
$$

Proof. Equation (6.6) shows we can regard $G_{22}^{(-1)}$ as a one-dimensional kernel with values in $\operatorname{End}(V)$, where $V=\operatorname{Ker}\left(\Delta_{H}\right)$. Consider the boundary conditions given by compatibility conditions with adjacent faces. 
On $B_{12}$ and $B_{11}$ the left model operator is $\Delta_{\bar{H}}$, given in local coordinates by (3.18). Since $G_{12}^{(-1)}$ and $G_{11}^{(-1)}$ are bounded, they are constant in $r=$ $\sinh ^{-1}(x / \epsilon)$, the variable across $B_{12}$ and $B_{11}$, so they give trivial matching conditions across $s=0$ for $G_{22}^{(-1)}$, as in the proof of Proposition 3.8. We similarly get trivial matching conditions across $B_{21}, B_{11}$ at $s^{\prime}=0$ and across $B_{33}$ at $s=s^{\prime}$. Hence $G_{22}^{(-1)}$ is continuous on $[-1,1]_{s} \times[-1,1]_{s^{\prime}}$. At $s=$ $\pm 1, G_{22}^{(-1)}$ matches with $G_{02}^{(-1)}$ which is bounded and $\operatorname{Ker}\left(\Delta_{\bar{M}}\right) \times \operatorname{Ker}\left(\Delta_{H}\right)$ valued; hence $G_{22}^{(-1)} \uparrow s= \pm 1$ takes values in $\Lambda_{ \pm}^{D} \otimes V \equiv \operatorname{Hom}\left(V, \Lambda_{ \pm}^{D}\right)$ and similarly $G_{22}^{(-1)}\left\lceil s^{\prime}= \pm 1\right.$ takes values in $V \otimes \Lambda_{ \pm}^{D} \equiv \operatorname{Hom}\left(\Lambda_{ \pm}^{D}, V\right)$.

In the interior the derivatives $\partial_{s} G_{22}^{(-1)}, \partial_{s^{\prime}} G_{22}^{(-1)}$ match across $B_{12}, B_{21}$. The derivative $\partial_{s} G_{22}^{(-1)}$ at $s= \pm 1$ matches, as in the proof of Proposition 3.8, with the $\xi^{-1}$ term of $G_{02}^{(0)}$ at $\xi=0$, so $\partial_{s} G_{22}^{(-1)}\left\lceil s= \pm 1 \in \operatorname{Hom}\left(V, \Lambda_{ \pm}^{N}\right)\right.$ and similarly $\partial_{s^{\prime}} G_{22}^{(-1)} \uparrow s^{\prime}= \pm 1 \in \operatorname{Hom}\left(\Lambda_{ \pm}^{N}, V\right)$. Across $B_{33}$, compatibility between $G_{22}^{(-1)}$ and $G_{33}^{(0)}$ requires that at the intersection of $B_{22}$ and $B_{33}$, $\left(\partial_{s^{\prime}}-\partial_{s}\right) G_{22}^{(-1)}$ matches the $\log \left(x^{\prime} / x\right)$ coefficient of $G_{33}^{(0)}$. The model equation for $G_{33}^{(0)}$ is $\Delta_{\bar{H}} G_{33}^{(0)}=\operatorname{Id}_{\bar{H}}$, so $G_{33}^{(0)}$ is given by

$$
\begin{aligned}
G_{33}^{(0)}=\sum_{j=1}^{\infty} \frac{e^{-\sigma_{j}\left|\log \left(x^{\prime} / x\right)\right|}}{2 \sigma_{j}} & \operatorname{proj}_{V_{j}}- \\
& -\frac{1}{2}\left|\log \frac{x^{\prime}}{x}\right| \operatorname{proj} V+A(s)+B(s) \log \frac{x^{\prime}}{x}
\end{aligned}
$$

where $V_{j}$ is the $j$-th nonzero eigenspace of $\Delta_{H}$ with eigenvalue $\sigma_{j}^{2}$, and $A(s)$, $B(s)$ are $\operatorname{End}(V)$-valued functions of $s$. There is a jump of $\operatorname{Id} \in \operatorname{End}(V)$ in the coefficient of $\log \left(x^{\prime} / x\right)$ between the two sides of $B_{33}$. Hence $\left(\partial_{s^{\prime}}-\partial_{s}\right) G_{22}^{(-1)}$ has a jump of Id across $s=s^{\prime}$. These conditions on $G_{22}^{(-1)}$ uniquely determine that $G_{22}^{(-1)}=\mathfrak{T}\left(\bar{K}\left(s, s^{\prime}, z\right)\right)$, the kernel of the one-dimensional resolvent studied in section 5 .

Indeed, not only does this argument yield $G_{22}^{(-1)}$, it gives us all the $G_{m n}^{(-1)}$. Compatibility of $G_{m n}^{(-1)}$ with $G_{22}^{(-1)}$ requires that $G_{m n}^{(-1)}=\mathfrak{T}\left(K_{m n}^{(-1)}\right)$ for all $m n$. The compatibility of these terms itself follows from the compatibility on $X_{\mathrm{Ls}}^{2}([-1,1])$. 


\subsection{Terms of order (ias $\epsilon)^{0}$.}

To find the next term in the Taylor series at each face, we start with the faces $B_{00}, B_{11}$ and $B_{33}$ whose normal equations, (6.3) - (6.5) have 'forcing terms' on the right hand side. Let us begin with $G_{00}^{(0)}$. By (6.3) and the discussion in section 6.2 , this term takes the form

$$
G_{00}^{(0)}=\operatorname{Res} \frac{(0)}{M}+\text { terms in } \operatorname{Ker}\left(\Delta_{\bar{M}}\right) \otimes \operatorname{Ker}\left(\Delta_{\bar{M}}\right) .
$$

The regular part of the resolvent can be calculated from (6.12) - (6.14). Its leading behaviour is, at the front face:

$$
\begin{array}{r}
\frac{1}{2}\left(-\left|\log \frac{x^{\prime}}{x}\right| \operatorname{Id}+\left(\log x+\log x^{\prime}\right) S_{J K}(0) \phi_{J}(y) \phi_{K}\left(y^{\prime}\right)-i \phi_{J}(y) \phi_{K}\left(y^{\prime}\right) S_{J K}^{(1)}\right) \\
= \begin{cases}-\log x \operatorname{proj} \Lambda_{+}^{N}+\log x^{\prime} \operatorname{proj} \Lambda_{+}^{D}-\frac{i}{2} \phi_{J}(y) \phi_{K}\left(y^{\prime}\right) S_{J K}^{(1)}, & \frac{x^{\prime}}{x}<1 \\
\log x \operatorname{proj} \Lambda_{+}^{D}-\log x^{\prime} \operatorname{proj} \Lambda_{+}^{N}-\frac{i}{2} \phi_{J}(y) \phi_{K}\left(y^{\prime}\right) S_{J K}^{(1)}, & \frac{x^{\prime}}{x}>1\end{cases}
\end{array}
$$

at the left boundary:

$$
\begin{aligned}
& \frac{1}{2}\left(\log x \phi_{J}(y) \Phi_{J}^{(0)}\left(p^{\prime}\right)-i \phi_{J}(y) \Phi_{J}^{(1)}\right) \\
= & \frac{1}{2}\left(\log x \phi_{j}(y) \Phi_{j}^{(0)}\left(p^{\prime}\right)-i \phi_{\alpha}(y) \Phi_{\alpha}^{(1)}\left(p^{\prime}\right)-i \phi_{j}(y) S_{j k}^{(1)} \Phi_{k}^{(0)}\left(p^{\prime}\right)\right),
\end{aligned}
$$

and at the right boundary:

$$
\begin{aligned}
& \frac{1}{2}\left(\log x^{\prime} \Phi_{J}^{(0)}(p) \phi_{J}\left(y^{\prime}\right)-i \Phi_{J}^{(0)} \phi_{J}\left(y^{\prime}\right)\right) \\
= & \frac{1}{2}\left(\log x^{\prime} \Phi_{j}^{(0)}(p) \phi_{j}\left(y^{\prime}\right)-i \Phi_{\alpha}^{(1)}(p) \phi_{\alpha}\left(y^{\prime}\right)-i S_{j k}^{(1)} \Phi_{k}^{(0)}(y) \phi_{j}\left(y^{\prime}\right)\right) .
\end{aligned}
$$

The relation between these expressions at the front face and at the left and right boundaries is perhaps not immediately evident since there only appears $S_{j k}^{(1)}$, with small roman indices, in the last two. The reason is that the $S_{\alpha \beta}^{(1)}$ piece is contained in the $\Phi_{\alpha}^{(1)}$ term, which may have a part bounded up to the boundary 'beneath' the principal, logarithmically increasing one. Since $\partial_{\lambda} S_{J K}(0)$ is block diagonal with respect to the splitting $V=\Lambda_{+}^{D} \oplus \Lambda_{+}^{N}$, the other pieces $S_{i \alpha}^{(1)}, S_{\alpha i}^{(1)}$ are zero. The null space terms in (6.16) are 
determined by compatibility with $G_{22}^{(-1)}$. The one-dimensional model is

$$
\begin{aligned}
K_{00}^{(0)} & =(\operatorname{ias} \epsilon)^{-1}\left(\bar{K}\left(s, s^{\prime}, z\right)-\bar{K}(1,1, z)\right)\left\lceil B_{00}\right. \\
& =(\operatorname{ias} \epsilon)^{-1}\left((s-1) \partial_{s} \bar{K}(1,1, z)+\left(s^{\prime}-1\right) \bar{K}(1,1, z)\right) \uparrow B_{00} \\
& =\log x \partial_{s} \bar{K}(1,1, z)+\log x^{\prime} \partial_{s^{\prime}} \bar{K}(1,1, z) \\
& = \begin{cases}\log x\left(-\operatorname{proj} \Lambda_{+}^{N}+A^{*}(\bar{z})\right)+\log x^{\prime}\left(\operatorname{proj} \Lambda_{+}^{D}+A(z)\right) & \frac{x^{\prime}}{x}<1 \\
\log x\left(\operatorname{proj} \Lambda_{+}^{D}+A^{*}(\bar{z})\right)+\log x^{\prime}\left(-\operatorname{proj} \Lambda_{+}^{N}+A(z)\right) & \frac{x^{\prime}}{x}>1\end{cases}
\end{aligned}
$$

for $x, x^{\prime}>0 ;(5.2)$ has been used in the last two lines. The projection terms a.lready appear in (6.17) and the $A(z)$ terms take values in the null space terms which give compatibility with $K_{22}^{(-1)}$. Hence, defining

$$
\begin{aligned}
G_{00}^{(0)} & =\operatorname{Reg}_{\bar{M}}(0)+\mathfrak{T}\left(\log x A(z)+\log x^{\prime} A^{*}(\bar{z})\right) . \\
& =\operatorname{Reg}_{\bar{M}}(0)+A_{\alpha j}(z) \Phi_{\alpha}^{(1)}(p) \phi_{j}\left(y^{\prime}\right)+A_{j \alpha}^{*}(\bar{z}) \phi_{j}(y) \Phi_{\alpha}^{(1)}\left(p^{\prime}\right),
\end{aligned}
$$

$G_{00}^{(0)}$ and $G_{22}^{(-1)}$ are compatible. The same argument shows that $G_{00}^{(0)}$ and $G_{02}^{(-1)}, G_{20}^{(-1)}, G_{33}^{(-1)}$ are compatible.

We can use similar reasoning for $G_{33}^{(0)}$ and $G_{11}^{(0)}$. Using coordinates $\log \left(x^{\prime} / x\right), s$, ias $\epsilon$ near $B_{33}$, we have

$$
\begin{aligned}
K_{33}^{(0)} & =(\operatorname{ias} \epsilon)^{-1}\left(\bar{K}\left(s, s+\operatorname{ias} \epsilon \log \left(x^{\prime} / x\right), z\right)-\bar{K}(s, s, z)\right) \uparrow B_{11} \\
& =\log \left(x^{\prime} / x\right) \partial_{s^{\prime}} \bar{K}(s, s, z) \\
& =-\frac{1}{2}\left|\log \frac{x^{\prime}}{x}\right| \phi_{J}(y) \phi_{J}\left(y^{\prime}\right)+\log \frac{x^{\prime}}{x} D_{I J}(s, z) \phi_{I}(y) \phi_{J}\left(y^{\prime}\right),
\end{aligned}
$$

where this defines $D_{I J}$. Note that $D_{I J}( \pm 1, z)=\frac{1}{2}\left(\operatorname{proj} \Lambda_{ \pm}^{N}-\operatorname{proj} \Lambda_{ \pm}^{D}\right)+A^{*}(\bar{z})$. By construction, the transfer of this term is compatible with $G_{22}^{(-1)}, G_{11}^{(-1)}$ and $G_{00}^{(-1)}$. Referring to (6.15) and (6.17), we should also add a term to give compatibility with the $\partial_{\lambda} S$ terms in $G_{00}^{(0)}$. So define

$$
\begin{aligned}
G_{33}^{(0)} & =\operatorname{Res} \frac{(0)}{H}+\log \frac{x^{\prime}}{x} D_{I J}(s, z) \phi_{I}(y) \phi_{J}\left(y^{\prime}\right) \\
& -\frac{i}{2} \psi\left(2-s-s^{\prime}\right) e^{-i z\left(2-s-s^{\prime}\right)} \phi_{J}(y) S_{J K}^{(1)} \phi_{K}\left(y^{\prime}\right) .
\end{aligned}
$$

Here $\psi$ is a cutoff function, with support near 0 . The factor $e^{-i z\left(2-s-s^{\prime}\right)}$ may appear somewhat mysterious; it will be needed in the next section when 
we join these models with those constructed in Part I. The $\psi$ term ensures compatibility of $G_{33}^{(0)}$ with $G_{00}^{(0)}$ and does not affect the compatibility with any terms of order $(\text { ias } \epsilon)^{-1}$, since it has no part growing as $\rho_{p q}^{-1}$ at any adjacent face.

The $G_{11}^{(0)}$ term may be defined similarly except that no extra term is required as $B_{11}$ is disjoint from $B_{00}$. Using coordinates $r, r^{\prime}, y, y^{\prime}$, we have

$$
\begin{aligned}
K_{11}^{(0)} & =(\operatorname{ias} \epsilon)^{-1}\left(\bar{K}\left((\operatorname{ias} \epsilon) r,(\operatorname{ias} \epsilon) r^{\prime}, z\right)-\bar{K}(0,0, z)\right) \uparrow B_{11} \\
& =r \partial_{s} \bar{K}(0,0, z)+r^{\prime} \partial_{s^{\prime}} \bar{K}(0,0, z) \\
& =\left(r^{\prime}-r\right) \partial_{s^{\prime}} \bar{K}(0,0, z)+r \partial_{s} \bar{K}(0,0, z) \\
& =-\frac{1}{2}\left|r^{\prime}-r\right| \phi_{J}(y) \phi_{J}\left(y^{\prime}\right)+\left(r^{\prime}-r\right) D_{I J}(0, z) \phi_{I}(y) \phi_{J}\left(y^{\prime}\right)+r \partial_{s} \bar{K}(0,0, z) .
\end{aligned}
$$

With

$$
G_{11}^{(0)}=\operatorname{Res} \frac{(0)}{H}+\left(r^{\prime}-r\right) D_{I J}(0, z) \phi_{1}(y) \phi_{J}\left(y^{\prime}\right)+\mathfrak{T}\left(r \partial_{s} \bar{K}(0,0, z)\right)
$$

this model is compatible with all $G_{m n}^{(-1)}$, and with $G_{33}^{(0)}$.

Next consider $G_{02}^{(0)}$ and $G_{01}^{(0)}$. To satisfy compatibility with $G_{m n}^{(-1)}$, we must take

$$
G_{02}^{(0)}-\mathfrak{T}\left(K_{02}^{(0)}\right) \in C^{\infty}\left([-1,1]_{s^{\prime}} ; C^{\infty} \operatorname{Ker}\left(\Delta_{\bar{M}}\right) \otimes V^{*}\right) .
$$

To make this term compatible with $G_{00}^{(0)}$, let us define

$$
G_{02}^{(0)}=\mathfrak{T}\left(K_{02}^{(0)}\right)-\frac{i}{2} \psi\left(1-s^{\prime}\right) e^{-i z\left(1-s^{\prime}\right)} \Phi_{j}^{(0)}(p) S_{j k}^{(1)} \phi_{k}\left(y^{\prime}\right) .
$$

This is now compatible with all models defined above. Since the 'extra' term in $G_{02}^{(0)}$ is supported away from $G_{01}^{(0)}$, we can define

$$
G_{01}^{(0)}=\mathfrak{T}\left(K_{01}^{(0)}\right)
$$

and then $G_{01}^{(0)}$ is compatible with all terms already defined.

The model $G_{21}^{(0)}$ must be of the form

$$
G_{21}^{(0)}=\mathfrak{T}\left(K_{21}^{(0)}\right)+C^{\infty}\left([0, \pm 1]_{s} ; \operatorname{End}(V)\right)
$$

to satisfy its model equation and be compatible with all models of order (ias $\epsilon)^{-1}$. We also require compatibility with $G_{01}^{(0)}$ and $G_{11}^{(0)} \cdot \mathfrak{T}\left(K_{01}^{0}\right)$ is given 
by

$$
\begin{gathered}
\mathfrak{T}\left(K_{01}^{(0)}\right)=\mathfrak{T}\left((\operatorname{ias} \epsilon)^{-1}\left(\bar{K}\left(1-\xi^{-1}(\operatorname{ias} \epsilon),(\operatorname{ias} \epsilon) r^{\prime}, z\right)-\bar{K}(1,0, z)\right)\left\lceil B_{01}\right)\right. \\
=\mathfrak{T}\left(-\log x \partial_{s} \bar{K}(1, o, z)+r^{\prime} \partial_{s^{\prime}} \bar{K}(1,0, z)\right) .
\end{gathered}
$$

So $G_{01}^{(0)}$ has the form

$$
C_{\alpha I} \Phi_{\alpha}^{(1)}(p) \phi_{I}\left(y^{\prime}\right)+r^{\prime} E_{i J} \Phi_{i}^{(0)}(p) \phi_{J}\left(y^{\prime}\right) .
$$

Recall that $\Phi_{\alpha}^{(1)} \sim-i \log x \phi_{\alpha}+S_{\alpha \beta}^{(1)} \phi_{\beta}$ at $x=0$. We therefore define

$$
G_{21}^{(0)}=\mathfrak{T}\left(K_{21}^{(0)}\right)+\psi(1-s) e^{i z(1-s)} C_{\alpha I} S_{\alpha \beta}^{(1)} \phi_{\beta}(y) \phi_{1}\left(y^{\prime}\right) ;
$$

this now matches $G_{01}^{(0)}$ at $B_{01} \cap B_{21}$ and is compatible with $G_{11}^{(0)}$. The other models $G_{20}^{(0)}, G_{10}^{(0)}$ and $G_{12}^{(0)}$ are defined similarly.

\subsection{Terms of order (ias $\epsilon)$.}

These terms are only required for those faces $B_{m n}$ with $e\left(B_{m n}\right)=2$ (that is, for $d\left(B_{m n}\right) \geq 2$ ) and, as observed above, not for $B_{22}$. Let us start with $G_{02}^{(1)}$. To satisfy compatibility with $G_{m n}^{(-1)}$, we must have

$$
G_{02}^{(1)}-\mathfrak{T}\left(K_{02}^{(1)}\right) \in C^{\infty}\left([-1,0]_{s^{\prime}} \cup[0,1]_{s^{\prime}} ; \operatorname{Ker}^{-}\left(\Delta_{\bar{M}}\right) \otimes V^{*}\right),
$$

where $\operatorname{Ker}^{-}\left(\Delta_{\bar{M}}\right)$ is the direct sum of the bounded and logarithmically growing null space of $\Delta_{\bar{M}}$. For compatibility with $G_{00}^{(0)}$ and $G_{01}^{(0)}$, we may take the null space term to be zero; however let us add a term which will be required in the next section:

$$
G_{02}^{(1)}=\mathfrak{T}\left(K_{02}^{(1)}\right)+\frac{z}{2 i} \psi\left(1-s^{\prime}\right) e^{-i z\left(1-s^{\prime}\right)} \Phi_{\alpha}^{(2)}(p) \phi_{\alpha}\left(y^{\prime}\right) .
$$

(Recall that $\Phi_{\alpha}^{(2)}$ has zero leading, $(\log x)^{2}$ term. Hence there is no compatibility required between the last term and $G_{22}^{(-1)}$ as might at first appear.) We define $G_{20}^{(1)}$ similarly. The $G_{33}^{(1)}$ term may, in the same way, be taken to be $\mathfrak{T}\left(K_{33}^{(1)}\right)$ but again we add a term required in the next section:

$$
G_{33}^{(1)}=\mathfrak{T}\left(K_{33}^{(1)}\right)+\frac{z}{2 i} \psi\left(2-s-s^{\prime}\right) e^{-i z\left(2-s-s^{\prime}\right)} \phi_{J}(y) S_{J K}^{(2)} \phi_{K}\left(y^{\prime}\right) .
$$


Finally we define

$$
G_{m n}^{(1)}=\mathfrak{T}\left(K_{m n}^{(1)}\right), m n=12,21 .
$$

Checking compatibility of these terms is rather simple. As all the boundary hypersurfaces for which models of order (ias $\epsilon)^{1}$ need be defined are disjoint, they must be checked only against models of lower order. Then the comment made in the paragraph below (6.8) applies; it is straightforward to show that the models defined in this subsection are compatible with all those previously defined.

\subsection{Compatibility with the symbol.}

The diagonal singularity of $\left(\Delta_{\epsilon}-(\text { ias } \epsilon)^{2} z^{2}\right)$, is equal to that of $\Delta_{\epsilon}+$ $O\left((\operatorname{ias} \epsilon)^{2}\right)$. In our chosen coordinates, $\Delta_{\epsilon}$ is constant, to infinite order at the boundary, so compatibility requires that terms $G_{m m}^{(-1)}$ and $G_{33}^{(1)}$ are smooth, and the singularity of $G_{m m}^{(0)}$ is the symbolic inverse of that of $\Delta_{\epsilon} \uparrow B_{m m}$. This indeed holds because the restriction of $\Delta_{\epsilon}$ to $B_{00}$ (respectively $B_{11}$, $B_{33}$ ) is $\Delta_{\bar{M}}$ (resp. $\Delta_{\bar{H}}$ ) and $G_{00}^{(0)}$ (resp. $G_{11}^{(0)}, G_{33}^{(0)}$ ) are chosen to be, up to smoothing operators, the inverses of $\Delta_{\bar{M}}$ (resp. $\Delta_{\bar{H}}$ ).

It follows that one can construct a holomorphic family of Ls- $\psi$ dos $G(z) \in \Psi_{\mathrm{Ls}}^{-2,-1}(X)$ restricting to the models which we have defined and having the correct diagonal singularity. This completes our construction of the parametrix.

\subsection{From parametrix to resolvent.}

We have outlined already, in section 4.1, the process of getting the actual resolvent from our parametrix $G(z)$. We have now, for $z \in \mathbb{C} \backslash \mathbb{R}^{+}$, a holomorphic family $G(z)$ such that (6.1) holds. Let $-R=\Delta G-\operatorname{Id} \in \mathcal{I}_{R}$. Then by Lemma 4.5, we have

$$
(\mathrm{Id}-R)^{-1}=\mathrm{Id}+S, S \in \mathcal{I}_{R} .
$$

Hence

$$
\left(\Delta-(\operatorname{ias} \epsilon)^{2} z^{2}\right) G(z)(\operatorname{Id}+S(z))=\operatorname{Id}
$$

so by Theorem 4.2 ,

$$
\left(\Delta-(\operatorname{ias} \epsilon)^{2} z^{2}\right)^{-1}=G+G \cdot S \in \Psi_{\mathrm{Ls}}^{-2,-1}(X)+\Psi_{\mathrm{Ls}}^{-\infty, \mathcal{F}}(X)
$$

where $\mathcal{F}$ is natural (see Definition 2). 
In fact all coefficients of elements of the index family $\mathcal{F}$ with nonzero logarithmic behaviour are zero. To see this suppose that there is a nonzero logarithmic term $\rho_{m n}^{j} \log \rho_{m n}^{k} \cdot a$, where $a$ is a section defined on $B_{m n}$, occurring at power $j$, with $j$ minimal. Since there is no other terms lower in the Taylor series with logarithmic behaviour, $a$ must be annihilated by the reduced normal operators of $\Delta$ :

$$
R_{m}(\Delta) \cdot a=a \cdot R_{n}(\Delta)=0
$$

But the solutions to the model problems on each face have the property (with one exception) that they are either bounded up to each boundary $B_{m n} \cap B_{p q}$ of the face or blow up like $1 / \rho_{p q}$ there. The only exception is $B_{33}$, which has this property with respect to $B_{22}$ but not $B_{00}$ or $B_{11}$. In any case, there is some face $B_{p q} \neq B_{33}$ at which $a$ is nonzero, we have, by compatibility of Taylor series, a term on $B_{p q}$ which behaves as $\log \rho_{m n}$. This contradicts the assertion above that solutions to models problems do not have logarithmic behaviour at the boundary. Therefore no such term exists. It follows then that $\left(\Delta_{\epsilon}-(\operatorname{ias} \epsilon)^{2} z^{2}\right)^{-1} \in \Psi_{\mathrm{Ls}}^{-2,-1}(X)$.

\subsection{Near the discrete spectrum of $\mathrm{RN}(\Delta)$.}

Recall from Section 5 that $\bar{K}\left(s, s^{\prime}, z\right)=\left(D_{s}^{2}-z^{2}\right)^{-1}$ is meromorphic in $z$ with poles at the spectrum $0 \leq z_{0}^{2}<z_{1}^{2} \cdots$. We have already constructed the resolvent away from the discrete spectrum. In this subsection, we will construct the resolvent in a neighbourhood of one of the $z_{j}$; at first, we shall take $z_{j} \neq 0$.

Near $z_{j}$ the kernel $\bar{K}$ of section 5 has the behaviour

$$
\bar{K}\left(s, s^{\prime}, z\right)=\frac{\operatorname{proj} V_{j}}{2 z_{j}\left(z_{j}-z\right)}+\bar{K}_{0}\left(s, s^{\prime}, z\right)
$$

where $V_{j}$ is the $j$ th eigenspace of $\mathrm{RN}(\Delta)$ and $\bar{K}_{0}$ is holomorphic near $z_{j}$. We have

$$
\left(D_{s}^{2}-z^{2}\right) \bar{K}_{0}\left(s, s^{\prime}, z\right)=\mathrm{Id}-\frac{z_{j}+z}{2 z_{j}} \operatorname{proj} V_{j}
$$

so $\bar{K}_{0}$ is an inverse up to finite rank near $z_{j}$. 
Let us examine this on $[-1,1]_{x}$ with surgery metric $g_{\epsilon}=d x^{2} /\left(x^{2}+\epsilon^{2}\right)$. With $s=($ ias $\epsilon) \sinh ^{-1}(x / \epsilon)$ as in section 5 , and defining

$$
\begin{aligned}
K_{0}\left(s, s^{\prime}, z, \operatorname{ias} \epsilon\right) & =(\operatorname{ias} \epsilon)^{-1} \bar{K}_{0}\left(s, s^{\prime}, z\right)\left|d s d s^{\prime} \frac{d \operatorname{ilg} \epsilon}{(\operatorname{ilg} \epsilon)^{2}}\right|^{\frac{1}{2}} \\
E\left(s, s^{\prime}, \operatorname{ias} \epsilon\right) & =\operatorname{ias} \epsilon \operatorname{proj} V_{j}\left|d s d s^{\prime} \frac{d \operatorname{ilg} \epsilon}{(\operatorname{ilg} \epsilon)^{2}}\right|^{\frac{1}{2}},
\end{aligned}
$$

we have $\left(\Delta_{\epsilon}-(\operatorname{ias} \epsilon)^{2} z^{2}\right) K_{0}=\mathrm{Id}-\left(z_{j}+z\right) / 2 z_{j} E$ on $X_{\mathrm{Ls}}^{2}([-1,1])$. Returning to our manifold $M$, by Proposition 3.8 we can construct from $\bar{e} \in V_{j}$ an approximate small eigenfunction $e$ on $X_{\mathrm{Ls}}^{2}$ such that $\left(\Delta-(\text { ias } \epsilon)^{2} z_{j}^{2}\right) e \epsilon$ $\rho_{0}^{2} \rho_{1}^{2} \rho_{2}^{3} C^{\infty}\left(X_{\mathrm{Ls}} ; \Omega_{D}^{\frac{1}{2}}\left(X_{\mathrm{Ls}}\right)\right)$. Let $\left\{\bar{e}_{i}\right\}_{i=1}^{N_{j}}$ be an orthonormal basis of $V_{j}, N_{j}=$ $\operatorname{dim} V_{j}$, and define

$$
W=\operatorname{ias} \epsilon \sum_{i=1}^{N_{j}} e_{i}(p) e_{i}\left(p^{\prime}\right)\left|\frac{d(\mathrm{ilg} \epsilon)}{(\mathrm{ilg} \epsilon)^{2}}\right|^{\frac{1}{2}} \in \Psi_{\mathrm{Ls}, \mathrm{bdy}}^{-\infty, 0}(X) .
$$

Thus $W$ is a uniformly finite rank operator of rank $N_{j}$, with

$$
W_{m n}^{(k)}=\mathfrak{T}\left(E_{m n}^{(k)}\right)
$$

for $k \leq 1$.

The first step in the construction of our parametrix near $z_{j}$ is analogous to the construction of $K_{0}$, an inverse for $D_{s}^{2}-z^{2}$ orthogonal to the troublesome terms $e_{i}$. Here, we define the $G_{m n}^{(j)}$ as before, but using $\bar{K}_{0}$ instead of $\bar{K}$. Then we have, by comparing $G$ with $K_{0}$,

$$
\left(\Delta_{\epsilon}-(\operatorname{ias} \epsilon)^{2} z^{2}\right) G(z)=\mathrm{Id}+\frac{z_{j}+z}{2 z_{j}} W-R(z)
$$

with $W$ as above and $R \in \mathcal{I}_{R}$. Inverting Id $-R$ as before, we get

$$
\left(\Delta_{\epsilon}-(\operatorname{ias} \epsilon)^{2} z^{2}\right)(G(z)(\operatorname{Id}+S(z)))=\operatorname{Id}+\frac{z_{j}+z}{2 z_{j}} W(\operatorname{Id}+S(z))=\operatorname{Id}-W^{\prime}(z)
$$

where Id $+S=(\operatorname{Id}-R)^{-1}$. We now have an inverse up to the uniformly rank $N_{j}$ error term $W^{\prime}=\left(z_{j}+z\right) /\left(2 z_{j}\right) W(\operatorname{Id}+S)$. The null space of $\operatorname{Id}-W^{\prime}$ is contained in range $W=\operatorname{span}\left\{e_{i}\right\}$. Let

$$
W^{\prime}(z) e_{i}=a_{i k}(z) e_{k}
$$


where each $a_{i k}(z)$ is a holomorphic map from a neighbourhood of $z_{j}$ to $\mathcal{A}_{\text {phg }}^{\mathcal{G}}([0, \delta])$, where $\mathcal{G}$ is natural. The function

$$
q(\operatorname{ias} \epsilon, z)=\operatorname{det}\left(\delta_{i k}-a_{i k}(z)\right)
$$

is holomorphic in $z$ and polyhomogeneous conormal in ias $\epsilon$ with natural index set, and such that

$$
q(0, z)=\left(\frac{z-z_{j}}{2 z_{j}}\right)^{N_{j}} .
$$

For small ias $\epsilon, q$ has exactly $N_{j}$ zeroes near $z_{j}$ and does not vanish in some small annulus around $z_{j}$. Hence $q$ vanishes precisely at the eigenvalues of $\Delta$ corresponding to $z_{j}^{2} \in \operatorname{spec} \mathrm{RN}(\Delta)$. The inverse $\left(\mathrm{Id}-W^{\prime}\right)^{-1}=\mathrm{Id}+F$ is therefore meromorphic in $z$, in the sense that $q \cdot(\operatorname{Id}+F)$ is holomorphic, and conormal in ias $\epsilon$ with natural index set. The resolvent is

$$
\left(\Delta_{\epsilon}-(\operatorname{ias} \epsilon)^{2} z^{2}\right)^{-1}=G(z)(\operatorname{Id}+S(z))(\operatorname{Id}+F) \in(\operatorname{ias} \epsilon)^{-1} \Psi_{\mathrm{Ls}}^{-2, \mathcal{G}^{\prime}}(X)
$$

and is meromorphic in the same sense as $F$, with $\mathcal{G}^{\prime}$ natural.

As in the last subsection we can show that actually we can take $\mathcal{G}^{\prime}$ to be the $C^{\infty}$ index family. Indeed, we already know this is so in any region away from $z_{j}$. Also we may calculate the projector onto small eigenfunctions corresponding to $z_{j}$ by the contour integral

$$
\Pi_{j}=\frac{1}{2 \pi i} \int_{\mathcal{C}}\left(\Delta_{\epsilon}-(\text { ias } \epsilon)^{2} z^{2}\right)^{-1} 2 z d z
$$

where $\mathcal{C}$ is a small circle that encloses all the zeroes of $q$, for small ias $\epsilon$, and stays away from all eigenvalues corresponding to other $z_{k}$. The value is, by the results of the previous subsection, in $\Psi_{\mathrm{Ls}}^{-2,1}(X)$. Moreover, the full symbol of the Laplacian is holomorphic near $z_{j}$, so the singularity at $\Delta_{\mathrm{Ls}}$ is removed and the result is actually in $\Psi_{\mathrm{Ls}}^{-\infty,-1}(X)$. Hence we have shown that the projector onto small eigenvalues corresponding to one $z_{j}$ is smooth on the space $X_{\mathrm{Ls}}^{2}$ and that the resolvent itself, $\left(\Delta_{\epsilon}-(\operatorname{ias} \epsilon)^{2} z^{2}\right)^{-1}$, is smooth on $X_{\mathrm{Ls}}^{2}$ and meromorphic in $z$.

If $z_{0}=0$, the argument is the same, but some of the formulae must be modified to accommodate the presence of a double pole at $z=0$. Equation (6.24) should be replaced by

$$
\bar{K}\left(s, s^{\prime}, z\right)=\operatorname{proj} V_{j}\left(1+z^{-2}\right)+\bar{K}_{0}\left(s, s^{\prime}, z\right) .
$$


In equation (6.27), we must replace $\left(z_{j}+z\right) / 2 z_{j}$ by $\left(1+z^{2}\right)$. Finally, equation (6.28) is replaced by

$$
q(0, z)=z^{2 N} .
$$

\subsection{In the presence of $L^{2}$ null space.}

Let $\left\{\phi_{i}\right\}$ be an orthonormal basis for the $L_{b}^{2}$-null space of $\Delta_{\bar{M}}$ on $M_{+} U$ $M_{-}$(on half-densities). Lift the $\phi_{i}$ to smooth half-densities on $X_{\mathrm{Ls}}$, also denoted $\phi_{i}$, which vanish to infinite order at $B_{1}$ and $B_{2}$. This is possible since on the space $\bar{M}, \phi_{i}$ decays at least as some positive power at the boundary, so on $\bar{M}_{\log }$ it vanishes to infinite order. We can then form the finite rank operator $\Pi_{L^{2}}=\sum_{i} \phi_{i}(p) \phi_{i}\left(p^{\prime}\right)\left|\frac{d(\mathrm{ilg} \epsilon)}{(\operatorname{ilg} \epsilon)^{2}}\right|^{1 / 2}$ which restricts at $B_{00}$ to the projector onto the $L^{2}$ null space of $\Delta$.

We have, near $\lambda=0$,

$$
\left(\Delta-\lambda^{2}\right)^{-1}=\operatorname{Reg}_{\bar{M}}(0)-\lambda^{-2} \Pi_{L^{2}},
$$

with $\operatorname{Reg}_{\bar{M}}(0)$ regular near $\lambda=0$. Since $\lambda^{-2}=(\text { ias } \epsilon)^{-2} z^{-2}$, we expect a term looking like (ias $\epsilon)^{-2} z^{-2} \Pi_{L^{2}}$ in our resolvent. Hence, we will get a nonzero term $G_{00}^{(-2)}$ of order (ias $\left.\epsilon\right)^{-2} ;$ since $\Pi_{L^{2}}$ vanishes to infinite order at all other faces this should be the only term of order (ias $\epsilon)^{-2}$.

Define $G_{00}^{(-2)}=\Pi_{L^{2}}\left\lceil B_{00}\right.$, and let the other $G_{m n}^{(j)}$ terms be as above. The $G_{00}^{(-2)}$ term is not a good approximation to the term in the resolvent we expect, but it is a holomorphic term which has approximate range the projection onto the expected null space, which is what we need for the construction to work. Then instead of (6.27), we get

$$
\left(\Delta_{\epsilon}-(\operatorname{ias} \epsilon)^{2} z^{2}\right) G=\mathrm{Id}-\left(1+z^{2}\right)\left(W+\Pi_{L^{2}}\right)-R
$$

with $R$ in the parametrix-residual space, and $W$ the approximate projection onto small eigenvalues corresponding to $0 \in \mathrm{RN}(\Delta)$. The $z^{2} \Pi_{L^{2}}$ term comes from - (ias $\epsilon)^{2} z^{2}$ acting on $G_{00}^{(-2)}$ and Id $-\Pi_{L^{2}}$ is produced from $\Delta$ acting on $G_{00}^{(0)}=\operatorname{Reg}_{\bar{M}}(0)$. Inverting $(\mathrm{Id}-R)$, we get

$$
\left(\Delta_{\epsilon}-(\operatorname{ias} \epsilon)^{2} z^{2}\right) G(\operatorname{Id}+S)=\operatorname{Id}-\left(1+z^{2}\right)\left(W+\Pi_{L^{2}}\right)(\operatorname{Id}+S),
$$

where $(\operatorname{Id}+R)^{-1}=\operatorname{Id}+S$. Then $\left(1+z^{2}\right)\left(W+\Pi_{L^{2}}\right)(\operatorname{Id}+S)=Y^{\prime}$ is a finite rank operator which we treat like $W^{\prime}$ in the last subsection. The range of 
$Y^{\prime}$ is spanned by $e_{i}$ and $\phi_{i}$. Let $\delta_{k l}-a_{k l}$ be the matrix of Id $-Y^{\prime}$ relative to the basis above. Then

$$
q(\operatorname{ias} \epsilon, z)=\operatorname{det}\left(\delta_{k l}-a_{k l}(z)\right)
$$

is holomorphic in $z$ and polyhomogeneous conormal in ias $\epsilon$ with natural index set, and such that

$$
q(0, z)=z^{2 N},
$$

where $N$ here is the sum of the dimensions of the $L^{2}$ null space of $\Delta_{\bar{M}}$ and the null space of $\operatorname{RN}(\Delta)$. The inverse $\left(\operatorname{Id}-Y^{\prime}\right)^{-1}=\operatorname{Id}+F^{\prime}$ is therefore meromorphic in $z$, in the sense that $q \cdot\left(\mathrm{Id}+F^{\prime}\right)$ is holomorphic, and conormal in ias $\epsilon$ with natural index set. The resolvent is

$$
\left(\Delta_{\epsilon}-(\operatorname{ias} \epsilon)^{2} z^{2}\right)^{-1}=G(z)(\operatorname{Id}+S(z))\left(\operatorname{Id}+F^{\prime}\right) \in \Psi_{\mathrm{Ls}}^{-2,-2}(X)
$$

and is meromorphic in the same sense as $F^{\prime}$.

The results of this subsection and the previous one show that as $\epsilon \rightarrow 0$, the small eigenvalues approach 0 or one of the eigenvalues of $\mathrm{RN}(\Delta)$ or infinity, as claimed in Theorem 1.1.

\subsection{Very small eigenvalues.}

The projectors $\Pi_{j}$ onto the eigenspace corresponding to $z_{j}$ are smooth, that is, have a complete asymptotic expansion in powers of ias $\epsilon$ at each boundary hypersurface of $X_{\mathrm{Ls}}$. For the eigenvalues corresponding to $z=$ 0 , which will be referred to as 'very small eigenvalues', this expansion is particularly simple. Let $\sigma_{1}$ be the first nonzero eigenvalue of $\Delta_{H}$. Then

Proposition 6.3. The very small eigenvalues $\lambda_{i}$ vanish as a power of $\epsilon$ :

$$
\lambda_{i}(\epsilon)=o\left(\epsilon^{\tau}\right) \text { for all } \tau<\max \left(\sigma_{1}, 1\right),
$$

so in particular are rapidly vanishing in ias $\epsilon$.

Proof. Cappell, Lee and Miller prove a very similar estimate in [8]. As the notations of the two papers are rather different, we sketch the proof here.

By the min-max principle, it is sufficient to construct an independent set of surgery 'test functions' $\psi_{i}$, equal to the number of very small eigenvalues, satisfying the estimate

$$
\left\|\Delta_{\epsilon} \psi_{i}\right\| \leq o\left(\left(\epsilon^{\tau}\right)\right)\left\|\psi_{i}\right\| \text { for all } \tau<\max \left(\sigma_{1}, 1\right) .
$$


Let $\left\{\chi_{i}^{0}\right\}_{i=1}^{k}$ be a basis of $L^{2} \operatorname{Ker} \Delta_{\bar{M}}$ and let $\left\{\bar{\phi}_{i}^{0}\right\}_{i=k+1}^{N_{0}}$ be a basis of $\Lambda_{+}^{D} \cap \Lambda_{-}^{D}$. Let $\left\{\phi_{i}^{0}\right\}_{i=k+1}^{N_{0}}$ be the corresponding elements of the bounded null space of $\Delta_{\bar{M}}$. Then, by the results of of [22],

$$
\chi_{i}^{0} \in \mathcal{A}_{\mathrm{phg}}^{\mathcal{K}^{0}}(\bar{M}) \text { and } \phi_{i}^{0} \in C^{\infty}(M)+\mathcal{A}_{\mathrm{phg}}^{\mathcal{K}^{0}}(\bar{M})
$$

where $\inf \operatorname{Re} \mathcal{K}^{0} \geq \sigma_{1}$. Hence, $\chi_{i}^{0}, \phi_{i}^{0}$ extend to $\chi_{i}, \phi_{i} \in \mathcal{A}_{\text {phg }}^{\mathcal{K}}\left(X_{s}\right)$, on the original surgery space (3.2), such that $\chi_{i} \uparrow B_{1}=0, \phi_{i}\left\lceil B_{1}=\bar{\phi}_{i}^{0}\right.$ and with index family $\mathcal{K}\left(B_{1}\right)=\mathcal{K}^{0}$ or $0+\mathcal{K}^{0}$ and $\mathcal{K}\left(B_{0}\right)=0$. Then the model operators of $\Delta_{\epsilon}$ kill the top terms at $B_{0}$ and $B_{1}$ so therefore $\Delta_{\epsilon} \chi_{i}, \Delta_{\epsilon} \phi_{i} \in \mathcal{A}_{\mathrm{phg}}^{\mathcal{J}}\left(X_{s}\right)$, with index family $\mathcal{J}$ satisfying $\inf \operatorname{Re} \mathcal{J}\left(B_{1}\right) \geq \sigma_{1}$, $\inf \operatorname{Re} \mathcal{J}\left(B_{0}\right) \geq 1$. The estimate (6.29) follows.

\section{Full Resolvent.}

\subsection{Resolvent spaces.}

In this section we will unify the resolvent $\left(\Delta_{\epsilon}-(\operatorname{ias} \epsilon)^{2} z^{2}\right)^{-1}$, constructed in the last section, with the resolvent $\left(\Delta-\lambda^{2}\right)^{-1}, \lambda \in \mathbb{C} \backslash \mathbb{R}$, constructed in Part I.

At the level of parameters, ias $\epsilon$ and $z$ (or $\lambda=z \operatorname{ias} \epsilon$ ), these regions are united in the space

$$
X_{\mathrm{LsR}}^{0}=\left[X_{\mathrm{Ls}}^{0} \times \overline{\mathbb{C}}_{\lambda} ;\{0\}_{\mathrm{ilg} \epsilon} \times\{0\}_{\lambda}\right]
$$

the 'zero-resolvent space'. We use $\overline{\mathbb{C}}$, the complex numbers compactified with a circle at infinity, to stay in the class of compact manifolds with corners. At ilg $\epsilon=0$ there are two boundary hypersurfaces, $B^{\mathbb{C}}=[\overline{\mathbb{C}} ; 0]_{\lambda}$, a punctured complex plane and $B^{0}=\overline{\mathbb{C}}_{z}$, where $z=\lambda /$ ias $\epsilon$, a disc (hemisphere). We define single, double and triple spaces analogous to $X_{\mathrm{LsR}}^{2}$ :

$$
\begin{aligned}
X_{\mathrm{LsR}} & =\left[X_{\mathrm{Ls}} \times \overline{\mathbb{C}}_{\lambda} ; M_{1}\left(X_{\mathrm{Ls}}\right) \times\{0\}_{\lambda}\right] \\
X_{\mathrm{LsR}}^{2} & =\left[X_{\mathrm{Ls}}^{2} \times \overline{\mathbb{C}}_{\lambda} ; M_{1}\left(X_{\mathrm{Ls}}^{2}\right) \times\{0\}_{\lambda}\right] \\
X_{\mathrm{LsR}}^{3} & =\left[X_{\mathrm{Ls}}^{3} \times \overline{\mathbb{C}}_{\lambda} ; M_{1}\left(X_{\mathrm{Ls}}^{3}\right) \times\{0\}_{\lambda}\right]
\end{aligned}
$$


Then, by Lemma 2.11 , we have a commutative diagram of simple bfibrations:

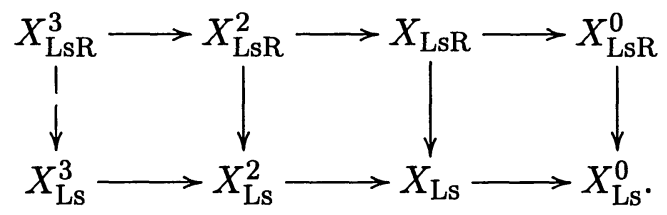

Each boundary hypersurface $B_{m n}$ of $X_{\mathrm{Ls}}^{2}$ lifts to two hypersurfaces in $X_{\mathrm{LsR}}^{2}$, one a blown up version of $B_{m n} \times \overline{\mathbb{C}}_{z}$, which we will denote $B_{m n}^{0}$ and the other a blown up version of $B_{m n} \times \overline{\mathbb{C}}_{\lambda}$, which will be denoted $B_{m n}^{\mathbb{C}}$. For $X_{\mathrm{LsR}}^{2}$ we define the degree of $B_{m n}^{0}\left(X_{\mathrm{LsR}}^{2}\right)$ and $B_{m n}^{\mathbb{C}}\left(X_{\mathrm{LsR}}^{2}\right)$ to be the same as that of $B_{m n}\left(X_{\mathrm{Ls}}^{2}\right)$. The next Lemma indicates the form of the degree density bundles of the resolvent spaces. Suppose that $Y$ is a manifold with corners with degrees, let $\mathcal{H}$ be a collection of boundary hypersurfaces of $Y$ and let $R$ be a product of defining functions over all elements of $\mathcal{H}$. Form the space $Z=[Y \times \mathbb{C} ; \mathcal{H} \times\{0\}]$, and let $\pi$ be the stretched projection down to $Y$. Define degrees for $Z$ by setting $d\left(\pi^{-1}(K \times \mathbb{C})\right)=d(K)$ and $d\left(\pi^{-1}(H \times\{0\})\right)=d(H)$ for $H \in \mathcal{H}$.

Lemma 7.1. There is an isomorphism

$$
\Omega_{D}\left(\left[Y \times \mathbb{C}_{\lambda} ; \mathcal{H} \times\{0\}\right]\right) \equiv \pi^{*} \Omega_{D}(Y) \otimes\left|\frac{d \lambda d \bar{\lambda}}{\lambda \bar{\lambda}+R^{2}}\right| .
$$

Proof. The result is clear away from $\lambda=0$, so suppose we are near $\lambda=0$ and near a corner of codimension $k$ in $Y \times \mathbb{C}$. Let $H_{1}, \ldots H_{k}$ be the boundary hypersurfaces forming the corner, with $H_{1} \ldots H_{l} \in \mathcal{H}$ let $r_{1}, \ldots r_{k}$ be boundary defining functions and suppose that $H_{1} \ldots H_{l}$ are blown up in that order, creating new hypersurfaces $H_{1}^{0} \ldots H_{l}^{0}$. Then the new boundary defining functions are $\rho_{i}^{0}, 1 \leq i \leq l$ where these are defined inductively by

$$
\begin{aligned}
\left(\rho_{i}^{0}\right)^{2} & =r_{i}^{2}+\left|\lambda_{i-1}\right|^{2}, \text { where } \\
\lambda_{0}=\lambda, \quad \lambda_{i} & =\frac{\lambda_{i-1}}{\rho_{i}^{0}} .
\end{aligned}
$$

Let $\rho_{i}^{\mathbb{C}}=r_{i} / \rho_{i}^{0}=\left(1+\left|\lambda_{i-1}\right|^{2}\right)^{-1 / 2}$ be a boundary defining function for the lift $H_{i}^{\mathbb{C}}$ of $H_{i} \times \mathbb{C}, 1 \leq i \leq l$. Notice that $\rho_{i}^{\mathbb{C}}=1$ on the lift of $H_{j} \times\{0\}$ for $j>i$. We first check (7.2) at $H_{1}^{0} \cap \cdots \cap H_{l}^{0} \cap \cdots \cap H_{k} \times \mathbb{C}$ and then at an 
intersection $H_{1}^{0} \cap \ldots \cap H_{m}^{0} \cap H_{m}^{\mathbb{C}} \cap \ldots$ Ignoring the $y$ factors in $Y$, which are irrelevant here, the right hand side of (7.2) is

$$
\prod_{i=1}^{k}\left(\frac{d r_{i}}{r_{i}^{d\left(H_{i}\right)+1}}\right) \frac{d \lambda d \bar{\lambda}}{|\lambda|^{2}+\left(r_{1} \ldots r_{k}\right)^{2}}
$$

After blowup of $H_{1} \times\{0\}$, we get

$$
\begin{gathered}
\prod_{i=2}^{k}\left(\frac{d r_{i}}{r_{i}^{d\left(H_{i}\right)+1}}\right) \frac{1}{\left(\rho_{1}^{0} \rho_{1}^{\mathbb{C}}\right)^{d\left(H_{i}\right)}} \frac{d r_{1}}{r_{1}} \frac{d \lambda d \bar{\lambda}}{|\lambda|^{2}+\left(r_{1} \ldots r_{k}\right)^{2}} \\
=\prod_{i=2}^{k}\left(\frac{d r_{i}}{r_{i}^{d\left(H_{i}\right)+1}}\right) \frac{1}{\left(\rho_{1}^{0} \rho_{1}^{\mathbb{C}}\right)^{d\left(H_{i}\right)}} \frac{d \rho_{1}^{0}}{\rho_{1}^{0}} \frac{\left(\rho_{1}^{0}\right)^{2} d \lambda_{1} d \bar{\lambda}_{1}}{\left(\rho_{1}^{0}\right)^{2}\left(\left|\lambda_{1}\right|^{2}+\left(\rho_{1}^{\mathbb{C}} r_{2} \ldots r_{k}\right)^{2}\right)} \text { at } H_{1}^{0} \\
=\prod_{i=2}^{k}\left(\frac{d r_{i}}{r_{i}^{d\left(H_{i}\right)+1}}\right) \frac{d \rho_{1}^{0}}{\left(\rho_{1}^{0}\right)^{d\left(H_{i}\right)+1}} \frac{1}{\left(\rho_{1}^{\mathbb{C}}\right)^{d\left(H_{1}\right)}} \frac{d \lambda_{1} d \bar{\lambda}_{1}}{\left|\lambda_{1}\right|^{2}+\left(\rho_{1}^{\mathbb{C}} r_{2} \ldots r_{k}\right)^{2}} .
\end{gathered}
$$

This is an expression of essentially the same form as (7.3) near (the lifts of) $H_{2} \times\{0\}, \ldots H_{k} \times\{0\}$ since $\rho_{1}^{\mathbb{C}}=1$ there. Hence applying this reasoning $l$ times gives

$$
\prod_{i=1}^{l}\left(\frac{d \rho_{i}^{0}}{\left(\rho_{i}^{0}\right)^{d\left(H_{i}\right)+1}\left(\rho_{i}^{\mathbb{C}}\right)^{d\left(H_{i}\right)}}\right) \prod_{i=l+1}^{k}\left(\frac{d r_{i}}{r_{i}^{d\left(H_{i}\right)+1}}\right) \frac{d \lambda_{l} d \bar{\lambda}_{l}}{\left|\lambda_{l}\right|^{2}\left(\rho_{1}^{\mathbb{C}} \ldots \rho_{l}^{\mathbb{C}} r_{l+1} \ldots r_{k}\right)^{2}}
$$

which is explicitly a $D$-density near $H_{1}^{0} \cap \cdots \cap H_{l}^{0} \cap \cdots \cap H_{k} \times \mathbb{C}$ since all the $\rho_{i}^{\mathbb{C}}, 1 \leq i \leq l$ are equal to 1 there.

We also need to check (7.2) near intersections $H_{m}^{0} \cap H_{m}^{\mathbb{C}}$. To do this, we only need to blow up $H_{1} \times\{0\} \ldots H_{m} \times\{0\}$, since all other blowups lie away from $H_{m}^{\mathbb{C}}$. Thus we can assume $m=l$. Then we have the boundary defining functions $\rho_{l}^{\mathbb{C}}$ or $\tilde{\rho}_{l}^{\mathbb{C}}=r_{l} / \lambda_{l-1}$ for $H_{l}^{\mathbb{C}}, \rho_{l}^{0}$ or $\lambda_{k-l}$ for $H_{l}^{0}$ and 
$\omega=\bar{\lambda}_{l-1} /\left|\lambda_{l-1}\right|$ as angular coordinate. We have

$$
\begin{array}{r}
\prod_{i=1}^{l-1}\left(\frac{d \rho_{i}^{0}}{\left(\rho_{i}^{0}\right)^{d\left(H_{i}\right)+1}\left(\rho_{i}^{\mathbb{C}}\right)^{d\left(H_{i}\right)}}\right) \prod_{i=l}^{k}\left(\frac{d r_{i}}{r_{i}^{d\left(H_{i}\right)+1}}\right) \frac{d \lambda_{l-1} d \bar{\lambda}_{l-1}}{\left|\lambda_{l-1}\right|^{2}+\left(\rho_{1}^{\mathbb{C}} \ldots \rho_{l-1}^{\mathbb{C}} r_{k}\right)^{2}} \\
=\prod_{i=1}^{l-1}\left(\frac{d \rho_{i}^{0}}{\left(\rho_{i}^{0}\right)^{d(H)_{i}+1}\left(\rho_{i}^{\mathbb{C}}\right)^{d\left(H_{i}\right)}}\right) \\
\times \prod_{i=l+1}^{k}\left(\frac{d r_{i}}{r_{i}^{d\left(H_{i}\right)+1}}\right) \frac{\lambda_{l-1} d \tilde{\rho}_{l}^{\mathbb{C}}}{\left(\tilde{\rho}_{l}^{\mathbb{C}} \lambda_{l-1}\right)^{d\left(H_{l}\right)+1}} \frac{\left|\lambda_{l-1}\right| d \lambda_{l-1} d \omega}{\left|\lambda_{l-1}\right|^{2}\left(1+\left(\rho_{1}^{\mathbb{C}} \ldots \rho_{l-1}^{\mathbb{C}} \tilde{\rho}_{l}^{\mathbb{C}}\right)^{2}\right)} \\
=\prod_{i=1}^{l-1}\left(\frac{d \rho_{i}^{0}}{\left(\rho_{i}^{0}\right)^{d\left(H_{i}\right)+1}\left(\rho_{i}^{\mathbb{C}}\right)^{d\left(H_{i}\right)}}\right) \\
\times \prod_{i=l+1}^{k}\left(\frac{d r_{i}}{r_{i}^{d\left(H_{i}\right)+1}}\right) \frac{d \tilde{\rho}_{l}^{\mathbb{C}} d \lambda_{l-1}}{\left(\tilde{\rho}_{h}^{\mathbb{C}} \lambda_{l-1}\right)^{d\left(H_{l}\right)+1}} \frac{\bar{\omega} d \omega}{1+\left(\rho_{1}^{\mathbb{C}} \ldots \rho_{l-1}^{\mathbb{C}} \tilde{\rho}_{l}^{\mathbb{C}}\right)^{2}} .
\end{array}
$$

Again, this is explicitly a $D$-density, so we have proved the lemma.

Taking $Y=X_{\mathrm{Ls}}^{j}, \mathcal{H}=M_{1}\left(X_{\mathrm{Ls}}^{j}\right)$, Lemma 7.1 yields

$$
\Omega_{D}\left(X_{\mathrm{LsR}}^{j}\right) \equiv \pi^{*} \Omega_{D}\left(X_{\mathrm{Ls}}^{j}\right) \otimes\left|\frac{d \lambda d \bar{\lambda}}{\lambda \bar{\lambda}+(\operatorname{ias} \epsilon)^{2}}\right|
$$

\subsection{Operator Calculus.}

To carry out the constructions of the previous section for the full resolvent space, we need the ingredients of that section - small pseudodifferential calculus, boundary terms, an iteratively-residual ideal - all extended to the 'full resolvent' setting. Here we can take advantage of the functorial nature of the constructions of section 4 , and simply observe that we obtain all these things from that section by replacing the spaces $X_{\mathrm{Ls}}^{j}$ with $X_{\mathrm{LsR}}^{j}$. We will denote the resulting spaces of LsR-pseudodifferential operators by

$$
\Psi_{\mathrm{Ls}, \mathrm{sml}}^{m}(X), \quad \Psi_{\mathrm{Ls}, \mathrm{bdy}}^{\mathcal{F}}(X) \quad \text { and } \Psi_{\mathrm{LsR}}^{m, \mathcal{F}}(X) .
$$

The composition properties of these operators are almost exactly as in section 4. Indeed, the combinatorics of the maps $\pi_{\mathrm{LsR}, O}^{3}: X_{\mathrm{LsR}}^{3} \rightarrow X_{\mathrm{LsR}}^{2}$ are easy to describe. For each boundary hypersurface $G$ (respectively $H$ ) of 
$X_{\mathrm{LS}}^{3}$ (resp. $X_{\mathrm{Ls}}^{2}$ ) there are two boundary hypersurfaces, $G^{\mathbb{C}}$ and $G^{0}$ (resp. $\left.H^{\mathbb{C}}, H^{0}\right)$ of $X_{\mathrm{LsR}}^{3}\left(\right.$ resp. $\left.X_{\mathrm{LsR}}^{2}\right)$ and if $\pi_{\mathrm{Ls}, O}^{3} G=H$ then

$$
\pi_{\mathrm{LsR}, O}^{3} G^{\mathbb{C}}=H^{\mathbb{C}} \text { and } \pi_{\mathrm{LsR}, O}^{3} G^{0}=H^{0} .
$$

Thus the mapping properties of the stretched projections on $X_{\mathrm{LsR}}^{j}$ are the 'same' as for $X_{\mathrm{Ls}}^{j}$. It follows that all the composition formulae in section 4 hold in the logarithmic resolvent calculus, and there is an iteratively-residual ideal $\mathcal{I}_{R}$ defined as in Lemma 4.4 which satisfies Lemma 4.5.

\subsection{Full Parametrix.}

We will look for a parametrix $G$ in the space $\lambda^{-2} \Psi_{\mathrm{LSR}}^{-2, \mathcal{F}}(X)$ such that

$$
\left(\Delta-\lambda^{2}\right) G-\operatorname{Id}=R \in \mathcal{I}_{R}
$$

away from the continuous spectrum, that is, in the set $X_{\mathrm{LsR}}^{0} \backslash\{\lambda \in \mathbb{R}, z=$ $\infty$. This includes the region where $z$ is finite, down to ias $\epsilon=0$, and joins the region $|z|$ large, $\delta<|\arg (z)|<\pi-\delta$ with $\lambda$ small, $\delta<|\arg (\lambda)|<\pi-\delta$.

We will construct the parametrix $G$ as before, as a finite Taylor series off the boundary hypersurfaces at ias $\epsilon=0$. We will continue to write $G_{m n}^{(j)}$ for the term at $B_{m n}$ of order (ias $\left.\epsilon\right)^{j}$ and write $G_{m n}^{(\mathbb{C})}$ for the term at $B_{m n}^{\mathbb{C}}$ of order 0 (there will be no other nonzero terms on the faces with $\lambda \neq 0$ ).

As in Chapter 6, we need to specify coordinates in order to define the higher order models. Let $\sigma$ denote the canonical density on $X_{\mathrm{LsR}}^{2}$. Recall the densities $\nu_{m n}$ defined in section 6.2. If $A_{m n}(z)\left(A_{m n}(\lambda)\right)$ is a D-half-density on $B_{m n}\left(X_{\mathrm{Ls}}^{2}\right)$ depending holomorphically on $z(\lambda)$, then write $A_{m n}(z)=$ $a\left(\mathcal{C}_{m n}, z\right) \nu_{m n}^{1 / 2}$ or $A_{m n}(\lambda)=a\left(\mathcal{C}_{m n}, \lambda\right) \nu_{m n}^{1 / 2}$, that is, express these half densities in terms of the coordinates comprising $\mathcal{C}_{m n}$ and the half density $\nu_{m n}^{1 / 2}$. Then

$$
\begin{aligned}
\tilde{A}_{m n}^{(0)} & =a\left(\mathcal{C}_{m n}, z\right) \sigma^{\frac{1}{2}} \text { or } \\
\tilde{A}_{m n}^{(\mathbb{C})} & =a\left(\mathcal{C}_{m n}, \lambda\right) \sigma^{\frac{1}{2}}
\end{aligned}
$$

represents an extension of $A_{m n}$ to a neighbourhood of $B_{m n}\left(B_{m n}^{\mathbb{C}}\right) \subset X_{\mathrm{LsR}}^{2}$. We then define higher order models according to (6.2).

The appropriate models for nonzero $\lambda$ can be read off from the parametrix constructed in Part I. Lifting their results to $X_{\mathrm{LsR}}^{2}$, we have

$$
\begin{aligned}
& G_{00}^{(\mathbb{C})}=\left(\Delta_{\bar{M}}-\lambda^{2}\right)^{-1} \\
& G_{11}^{(\mathbb{C})}=\left(\Delta_{\bar{H}}-\lambda^{2}\right)^{-1} \\
& G_{33}^{(\mathbb{C})}=\left(\Delta_{\bar{H}}-\lambda^{2}\right)^{-1}
\end{aligned}
$$


and all others are zero. Indeed, it is shown in Part I that the error in this parametrix is conormal of positive order on the original surgery space $X_{s}^{2}$, and so after logarithmic blowup vanishes to infinite order at all other boundary hypersurfaces. One can also derive these models directly on the logarithmic resolvent space, but that would conceal the simplicity of the results. We have defined the $G_{m n}^{(j)}$ in the previous section, so as to satisfy the model operator equations and compatibility amongst themselves. We need to check that the two sets of models are compatible. As explained in section 6 , for $G_{22}^{(0)}$ and $G_{22}^{(1)}$ we only need to check that adjacent faces are $\operatorname{Ker}\left(\Delta_{\bar{H}}\right) \otimes \operatorname{Ker}\left(\Delta_{\bar{H}}\right)$-valued and compatible amongst themselves; it is not necessary to write down explicit models for these terms.

Compatibility with $G_{00}^{(\mathbb{C})} \quad$ The face $B_{00}^{\mathbb{C}}$ intersects $B_{00}^{0}, B_{20}^{0}, B_{22}^{0}, B_{33}^{0}$ and $B_{02}^{0}$. Consider compatibility with $G_{22}^{(-1)}$. In the interior of the intersection, we may take boundary defining functions $\lambda$ for $B_{22}^{0}$ and $1 / z$ for $B_{00}^{\mathbb{C}}$, and coordinates $\lambda \log x, \operatorname{ilg}\left(x^{\prime} / x\right) / \operatorname{ilg} x, y$ and $y^{\prime}$ along the intersection. We refer back to $(6.8)$ for the compatibility condition. We calculate from (6.12) that $\left(G_{00}^{(\mathbb{C})}\right)_{22,-1}$ is

$$
\frac{1}{2 i}\left(\left(x^{\prime} / x\right)^{ \pm i \lambda} \phi_{J}(y) \phi_{J}\left(y^{\prime}\right)+x^{i \lambda} x^{i \lambda} \phi_{J}(y) S_{J K}(0) \phi_{K}\left(y^{\prime}\right)\right),
$$

and from (5.10), $\left(G_{22}^{(-1)}\right)_{00, \mathbb{C}, 1}$ is given by

$$
\frac{e^{-i z\left|s-s^{\prime}\right|}+e^{-i z\left|s-\left(2-s^{\prime}\right)\right|}}{2 i} \operatorname{proj} \Lambda_{ \pm}^{D}+\frac{e^{-i z\left|s-s^{\prime}\right|}-e^{-i z\left|s-\left(2-s^{\prime}\right)\right|}}{2 i} \operatorname{proj} \Lambda_{ \pm}^{N} .
$$

These agree since $S(0)=\operatorname{proj} \Lambda_{ \pm}^{D}-\operatorname{proj} \Lambda_{ \pm}^{N}$ and $e^{-i z\left|s-s^{\prime}\right|}=\left(x^{\prime} / x\right)^{ \pm i \lambda}$ and $e^{-i z\left|s-\left(2-s^{\prime}\right)\right|}=x^{i \lambda} x^{i \lambda}$.

Next consider compatibility with $G_{02}^{(j)}$. We may use boundary defining functions $\lambda$ for $B_{02}^{0}$ and $1 / z$ for $B_{00}^{\mathbb{C}}$, and coordinates $\lambda \log x^{\prime}$, ilg $x, y$ and $y^{\prime}$ along the intersection. Let us do the calculation at $s=s^{\prime}=1$. The calculation for the compatibility of $G_{00}^{(\mathbb{C})}$ and $G_{02}^{(-1)}$ is very similar to that above, so we omit it. To check compatibility of $G_{00}^{(\mathbb{C})}$ and $G_{02}^{(0)}$, we compute from (6.14)

$$
\left(G_{00}^{\mathbb{C}(0)}\right)_{22,0}=\frac{i}{2} e^{i \lambda \log x^{\prime}} \Phi_{J}^{(1)}(x, y) \phi_{J}\left(y^{\prime}\right)
$$


and from (5.10) and (6.21)

$$
\begin{aligned}
\left(G_{02}^{(0)}\right)_{00,0} & =\mathfrak{T}\left(K_{02}^{(0)}\right)+\frac{i}{2} \psi\left(\left(1-s^{\prime}\right) / 2\right) e^{-i z\left(1-s^{\prime}\right)} \Phi_{j}^{(1)}(p) S_{j k}^{(1)} \phi_{k}\left(y^{\prime}\right) \\
& =\mathfrak{T}\left(\partial_{s} \bar{K}(1,1-0, z)\right)+\frac{i}{2} e^{-i z\left(1-s^{\prime}\right)} \Phi_{j}^{(1)}(p) S_{j k}^{(1)} \phi_{k}\left(y^{\prime}\right) \\
& =\frac{1}{2} e^{-i z\left(1-s^{\prime}\right)} \Phi_{\alpha}^{(1)} \phi_{\alpha}+\frac{i}{2} e^{-i z\left(1-s^{\prime}\right)} \Phi_{j}^{(1)}(p) S_{j k}^{(1)} \phi_{k}\left(y^{\prime}\right)
\end{aligned}
$$

which agree. To check compatibility of $G_{00}^{(\mathbb{C})}$ and $G_{02}^{(1)}$, we compute

$$
\begin{gathered}
\left(G_{00}^{(\mathbb{C})}\right)_{22,1}=\frac{1}{2 i} e^{i \lambda \log x^{\prime}} \Phi_{J}^{(2)}(p) \phi_{J}\left(y^{\prime}\right) \\
\left(G_{02}^{(0)}\right)_{00, \mathbb{C},-1}=\left(\mathfrak{T}\left(K_{02}^{(1)}\right)\right)_{00, \mathbb{C},-1}+\frac{1}{2 i} e^{-i z\left(1-s^{\prime}\right)} \Phi_{\alpha}^{(2)}(p) \phi_{\alpha}\left(y^{\prime}\right) \\
=\frac{1}{2 i} e^{-i z\left(1-s^{\prime}\right)} \Phi_{j}^{(2)}(p) \phi_{j}\left(y^{\prime}\right)+\frac{1}{2 i} e^{-i z\left(1-s^{\prime}\right)} \Phi_{\alpha}^{(2)}(p) \phi_{\alpha}\left(y^{\prime}\right)
\end{gathered}
$$

using (5.10) again, which agrees.

Checking the compatibility of $G_{00}^{(\mathbb{C})}$ and $G_{33}^{(j)}$ requires calculations very similar to those above, so we omit the details. Let us check the compatibility of $G_{00}^{(\mathbb{C})}$ and $G_{00}^{(j)}$. Again we use $\lambda$ and $1 / z$ as boundary defining functions. Then

$$
\left(G_{00}^{(\mathbb{C})}\right)_{00,-1}=\operatorname{Res} \frac{(-1)}{M}=-i \operatorname{proj}\left\{v \mid \Delta_{\bar{M}} v=0, v \text { bounded }\right\}
$$

as remarked after (6.9), and

$$
\left(G_{00}^{(-1)}\right)_{00, \mathbb{C}, 1}=\mathfrak{T}\left((\bar{K}(1,1, z))_{00, \mathbb{C}, 1}\right)=\mathfrak{T}\left(-i \operatorname{proj} \Lambda_{ \pm}^{D}\right)
$$

by (5.10), which agrees. For $j=0$ we have

$$
\left(G_{00}^{(\mathbb{C})}\right)_{00,0}=\operatorname{Res} \frac{(0)}{M}
$$

and from (6.18)

$$
\left(G_{00}^{(0)}\right)_{00, \mathbb{C}, 0}=\operatorname{Res} \frac{(0)}{M}
$$

since $A(z)$ is rapidly decreasing in $z$.

Compatibility with $G_{33}^{(\mathbb{C})} \quad$ The face $B_{33}^{\mathbb{C}}$ intersects $B_{22}^{0}$ and $B_{33}^{0}$. In the interior of $B_{33}^{\mathbb{C}} \cap B_{22}^{0}$ we may use boundary defining functions $\lambda, 1 / z$ and 
coordinates $\lambda \log \left(x^{\prime} / x\right),\left(s+s^{\prime}\right) / 2, y, y^{\prime}$. We need check compatibility only with $G_{22}^{(-1)}$.

For $G_{33}^{(\mathbb{C})}$ we have an explicit expression in terms of the eigenvalues $\sigma_{j}$ and eigenspaces $V_{j}$ of $\Delta_{H}$ (where $\sigma_{0}=0$ and $V_{0}=V$ ):

$$
G_{33}^{(\mathbb{C})}=\left(\Delta_{\bar{H}}-\lambda^{2}\right)^{-1}=\sum_{j=0}^{\infty} \frac{e^{-\sqrt{\sigma_{j}^{2}-\lambda^{2}}\left|\log \left(x^{\prime} / x\right)\right|}}{2 \sqrt{\sigma_{j}^{2}-\lambda^{2}}} \operatorname{proj}_{V_{j}} .
$$

Hence

$$
\left(G_{33}^{(\mathbb{C})}\right)_{22,-1}=\frac{e^{-i \lambda\left|\log \left(x^{\prime} / x\right)\right|}}{2 i} \operatorname{proj}_{V}
$$

and by (5.10) and (5.9)

$$
\left(G_{22}^{(-1)}\right)_{33, \mathbb{C}, 1}=\frac{e^{-i z\left|s-s^{\prime}\right|}}{2 i} \operatorname{proj}_{V}
$$

which agree. (Note that, in (5.10), $e^{-i z\left(2-s-s^{\prime}\right)}$ and $e^{-i z\left(-2+s+s^{\prime}\right)}$ are rapidly vanishing at $B_{33}^{\mathbb{C}}$.)

We next check compatibility of $G_{33}^{(\mathbb{C})}$ and $G_{33}^{(j)}$. Again we use boundary defining functions $\lambda, 1 / z$. Then from (7.6) we calculate

$$
\begin{aligned}
\left(G_{33}^{(\mathbb{C})}\right)_{33,-1} & =\frac{\operatorname{proj}_{V}}{2 i} \\
\left(G_{33}^{(\mathbb{C})}\right)_{33,0} & =\operatorname{Res} \frac{(0)}{H} \\
\left(G_{33}^{(\mathbb{C})}\right)_{33,1} & =-\frac{\left(\log \left(x^{\prime} / x\right)\right)^{2}}{4 i} \operatorname{proj}_{V} .
\end{aligned}
$$

From (5.10) and (5.9), again observing that $e^{-i z\left(2-s-s^{\prime}\right)}$ is rapidly vanishing at $B_{33}^{\mathbb{C}}$, we see that the first one agrees with $\left(G_{33}^{(-1)}\right)_{33, \mathbb{C}, 1}$. From $(6.20)$, since $D(s, z)$ vanishes rapidly as $|z| \rightarrow \infty$, the second line agrees with $\left(G_{33}^{(0)}\right)_{33, \mathbb{C}, 0}$. Finally we calculate $\left(G_{33}^{(1)}\right)_{33, \mathbb{C},-1}$. Using coordinates as in (6.19), we have

$$
\left(G_{33}^{(1)}\right)_{33, \mathbb{C},-1}=\mathfrak{T}\left(\left(K_{33}^{(1)}\right)_{33, \mathbb{C},-1}\right)
$$

and

$$
\begin{aligned}
\left(K_{33}^{(1)}\right)_{33, \mathbb{C}, 1}=\left(\frac{e^{-i z(\operatorname{ias} \epsilon) \log \left(x^{\prime} / x\right)}}{2 i z} \operatorname{proj} V+O\left(|z|^{-\infty}\right)\right)_{33, \mathbb{C}, 1} \\
=\frac{-\left(\log \left(x^{\prime} / x\right)^{2}\right.}{4 i} \operatorname{proj} V
\end{aligned}
$$


which checks with the third line of (7.7).

Compatibility with $G_{11}^{(\mathbb{C})} \quad$ The checking of these compatibility conditions follows the same lines, but is considerably simpler; we leave these as an exercise for the reader.

Compatibility with the diagonal singularity We showed in the last section that the models $G_{j}^{(m n)}$ were compatible with the diagonal singularity. On the faces $B_{m m}^{\mathbb{C}}$, the singularities of $\Delta_{\epsilon}\left\lceil B_{m m}=\Delta_{\bar{M}}\right.$ and $\Delta_{\bar{H}}$ are equal to infinite order after logarithmic blowup, so these models are consistent with the singularity too. This is just lifting the compatibility result from Part I to this space.

It follows that one can construct a parametrix $G$ restricting to all the given models and compatible with diagonal singularity.

\subsection{Full Parametrix to Full Resolvent.}

This is done in exactly the same way as in section 6 . We may also argue as before to show that the resolvent is smooth away from the diagonal. Let us summarize the results of sections 6 and 7 :

Theorem 7.2. The resolvent family $\operatorname{Res}(\lambda)=\left(\Delta-\lambda^{2}\right)^{-1}$ lifts to the resolvent double space to an element of $\Psi_{\mathrm{LsR}}^{-2,0}(X)+\lambda^{-2} \Psi_{\mathrm{LsR}}^{-\infty, 0}(X)$ away from the continuous spectrum $\{\lambda$ real, $|z|=\infty\}$. The leading terms at each boundary hypersurface are:

$$
\begin{aligned}
\operatorname{Res}_{m n}^{(-1)} & =\mathfrak{T}\left(\left(\operatorname{RN}(\Delta)-z^{2}\right)^{-1}\right) \\
\operatorname{Res}_{00}^{(-2)} & =\operatorname{proj}_{L^{2}} \\
\operatorname{Res}_{00}^{(\mathbb{C})} & =\left(\Delta_{\bar{M}}-\lambda^{2}\right)^{-1} \\
\operatorname{Res}_{33}^{(\mathbb{C})} & =\left(\Delta_{\bar{H}}-\lambda^{2}\right)^{-1} \\
\operatorname{Res}_{11}^{(\mathbb{C})} & =\left(\Delta_{\bar{H}}-\lambda^{2}\right)^{-1} \text { and } \\
\operatorname{Res}_{m n}^{(\mathbb{C})} & \equiv 0 \text { otherwise. }
\end{aligned}
$$

Proof. The statement about smoothness follows from the argument given in section 6.7. The models $\operatorname{Res}_{m n}^{(j)}$ are given by $G_{m n}^{(j)}+(G \cdot S)_{m n}^{(j)}$. By Theorem 4.2, $G \cdot S$ has no order -1 terms up to the boundary, so the $\operatorname{Res}_{m n}^{(-1)}$ terms are given by $G_{m n}^{(-1)}=\mathfrak{T}\left(\left(\operatorname{RN}(\Delta)-z^{2}\right)^{-1}\right)$. At $B_{m n}^{\mathbb{C}}$ the parametrix is good 
to infinite order so the models of the resolvent agree with those of our parametrix.

\section{Heat Kernel.}

In this section we will define the logarithmic heat space and associated spaces, and prove Theorem 1.2. The heat kernel $e^{-t \Delta_{\epsilon}}$ is given by the operator valued contour integral

$$
\frac{i}{2 \pi} \int_{\Gamma} e^{-t \lambda^{2}}\left(\Delta_{\epsilon}-\lambda^{2}\right)^{-1} 2 \lambda d \lambda
$$

where $\Gamma \in \mathbb{C}$ is a contour that encloses (in a suitable sense) spec $\Delta_{\epsilon} \subset[0, \infty)$. In the spirit of this paper, we will perform the integral as a pushforward under a b-fibration between appropriate spaces. Then the Pushforward theorem will tell us that the result is polyhomogeneous and will give us the top order terms explicitly at each face. In the next subsection we will define the 'heat-resolvent space', lying above both the heat space and the resolvent space on which the integrand of (8.1) lies.

\subsection{The Heat Space and the Heat-Resolvent Space.}

The heat kernel for finite time $\left[0, C^{2}\right]$ has been treated in Part I. There are no changes that need to be made to treat the case when $\Delta_{H}$ has null space; the heat space $X_{\mathrm{hs}}^{2}$ defined in Part I is a suitable space to carry the heat kernel in general. It is in the behaviour of the heat kernel for large times that the situation is very different; in Part I the heat kernel was rapidly decreasing as $t \rightarrow \infty$ uniformly in $\epsilon$ (up to finite rank) but here this is no longer the case. In fact, the structure of the heat kernel near $t=\infty$ has the same degree of complexity as the resolvent near $\lambda=0$.

To define the heat space for large times $t \geq C^{2}$, let $\tau=\sqrt{t}$ and denote by $[C, \infty]_{\tau}$ the compactification of the interval $\{C \leq \tau<\infty\}$ with boundary defining function $\tau^{-1}$ at infinity. Then we define the logarithmic heat space to be, in the notation of Lemma 2.11 ,

$$
X_{\mathrm{LHs}}^{2,[C, \infty]}=\left[X_{\mathrm{Ls}}^{2} \times[C, \infty]_{\tau} ; M_{1}\left(X_{\mathrm{Ls}}^{2}\right) \times\{\tau=\infty\}\right] .
$$

Denote the lifts of $B_{m n} \times[C, \infty]_{\tau}$ by $B_{m n}\left(X_{\mathrm{LHs}}^{2,[C, \infty]}\right)$ or just $B_{m n}$ if this is unambiguous in context, and denote the result of blowing up $B_{m n} \times\{\infty\}$ by $B_{m n}^{\infty}$. Let the degrees of $B_{m n}\left(X_{\mathrm{LHs}}^{2,[C, \infty]}\right)$ and $B_{m n}^{\infty}$ be the same as that of 
$B_{m n}$, and define the degree of (the lift of) $X_{\mathrm{Ls}}^{2} \times\{\infty\}$ to be 0 and the degree of $X_{\mathrm{Ls}}^{2} \times\{C\}$ to be -1 . This means that the densities are smooth up to $\tau=C$, reflecting the fact that this is an artificial, and ignorable, boundary.

The heat-resolvent space is a space which maps down to both the heat space and the resolvent space, ie, it has both $t$ and $\lambda$ variables. It is not quite the space on which the integrand of (8.1) lies, since we also have to restrict to a contour of integration; this is done in the next subsection. To define the heat-resolvent space, let $\mathcal{B}_{j}\left(\mathcal{B}_{j}^{\infty}\right)$ be the set of $B_{m n}\left(B_{m n}^{\infty}\right)$ for $X_{\mathrm{LHs}}^{2}$ of degree $j$, for $j=1,2,3$. The heat-resolvent space is defined to be

$$
\begin{array}{r}
X_{\mathrm{LSHR}}^{2,[C, \infty]}=\left[X_{\mathrm{LHs}}^{2,[C, \infty]} \times \overline{\mathbb{C}}_{\lambda} ; \mathcal{B}_{3}^{\infty} \times\{0\}_{\lambda} ; \mathcal{B}_{3} \times\{0\}_{\lambda} ; \mathcal{B}_{2}^{\infty} \times\{0\}_{\lambda} ; \mathcal{B}_{2} \times\{0\}_{\lambda} ;\right. \\
\left.\mathcal{B}_{1}^{\infty} \times\{0\}_{\lambda} ; \mathcal{B}_{1} \times\{0\}_{\lambda}\right]
\end{array}
$$

The $\mathcal{B}_{3}^{\infty} \times\{0\}$ blowup separates the $\mathcal{B}_{3} \times\{0\}$ faces from the $\mathcal{B}_{2}^{\infty} \times\{0\}$ and the $\mathcal{B}_{1}^{\infty} \times\{0\}$ faces, and the $\mathcal{B}_{2}^{\infty} \times\{0\}$ blowup separates the $\mathcal{B}_{2} \times\{0\}$ faces from the $\mathcal{B}_{1}^{\infty} \times\{0\}$ faces. Hence, the heat-resolvent space is also given by

$$
\begin{aligned}
X_{\mathrm{LsHR}}^{2,[C, \infty]}=\left[X_{\mathrm{LHs}}^{2,[C, \infty]} \times \overline{\mathbb{C}}_{\lambda} ; \mathcal{B}_{3}^{\infty} \times\{0\}_{\lambda} ; \mathcal{B}_{2}^{\infty} \times\{0\}_{\lambda} ; \mathcal{B}_{1}^{\infty} \times\{0\}_{\lambda}\right. \\
\left.\mathcal{B}_{3} \times\{0\}_{\lambda} ; \mathcal{B}_{2} \times\{0\}_{\lambda} ; \mathcal{B}_{1} \times\{0\}_{\lambda}\right]
\end{aligned}
$$

We shall use both descriptions in order to lift maps to $X_{\mathrm{LsHR}}^{2,[C, \infty]}$. Let us denote the lifts of $B_{m n}\left(X_{\mathrm{LHs}}^{2,[C, \infty]}\right) \times\{0\}, B_{m n}\left(X_{\mathrm{LHs}}^{2,[C, \infty]}\right) \times \overline{\mathbb{C}}, B_{m n}^{\infty}\left(X_{\mathrm{LHs}}^{2,[C, \infty]}\right) \times$ $\{0\}$, and $B_{m n}^{\infty}\left(X_{\mathrm{LHs}}^{2,[C, \infty]}\right) \times \overline{\mathbb{C}}$ by $B_{m n}^{0}, B_{m n}^{\mathbb{C}}, B_{m n}^{\infty, 0}$ and $B_{m n}^{\infty, \mathbb{C}}$ respectively. Denoting the stretched projection $X_{\mathrm{LsHR}}^{2,[C, \infty]} \rightarrow X_{\mathrm{LHs}}^{2,[C, \infty]}$ by $\pi$, define degrees for the hypersurfaces of $X_{\mathrm{LSHR}}^{2,[C, \infty]}$ by letting $d(H)=d(\pi(H))$ for all $H \in$ $M_{1}\left(X_{\mathrm{LsHR}}^{2,[C, \infty]}\right)$.

These spaces have been defined with good mapping properties in mind. By Lemma 2.7, the b-fibration $X_{\mathrm{Ls}}^{2} \times[C, \infty]_{\tau} \rightarrow X_{\mathrm{Ls}}^{2}$ lifts to a b-fibration $\pi_{H}: X_{\mathrm{LHs}}^{2,[C, \infty]} \rightarrow X_{\mathrm{Ls}}^{2}$, and the b-fibration $X_{\mathrm{LHs}}^{2,[C, \infty]} \times \overline{\mathbb{C}}_{\lambda} \rightarrow X_{\mathrm{LHs}}^{2,[C, \infty]}$ lifts to a b-fibration $\pi_{\text {heat }}: X_{\mathrm{LsHR}}^{2,[C, \infty]} \rightarrow X_{\mathrm{LHs}}^{2,[C, \infty]}$. By Lemma 2.5, the b-fibration $X_{\mathrm{LHs}}^{2,[C, \infty]} \times \overline{\mathbb{C}} \rightarrow X_{\mathrm{Ls}}^{2} \times \overline{\mathbb{C}}$ lifts to a b-fibration $\pi_{\text {res }}: X_{\mathrm{LsHR}}^{2,[C, \infty]} \rightarrow X_{\mathrm{LsR}}^{2}$.

We then have the bundle isomorphisms: 


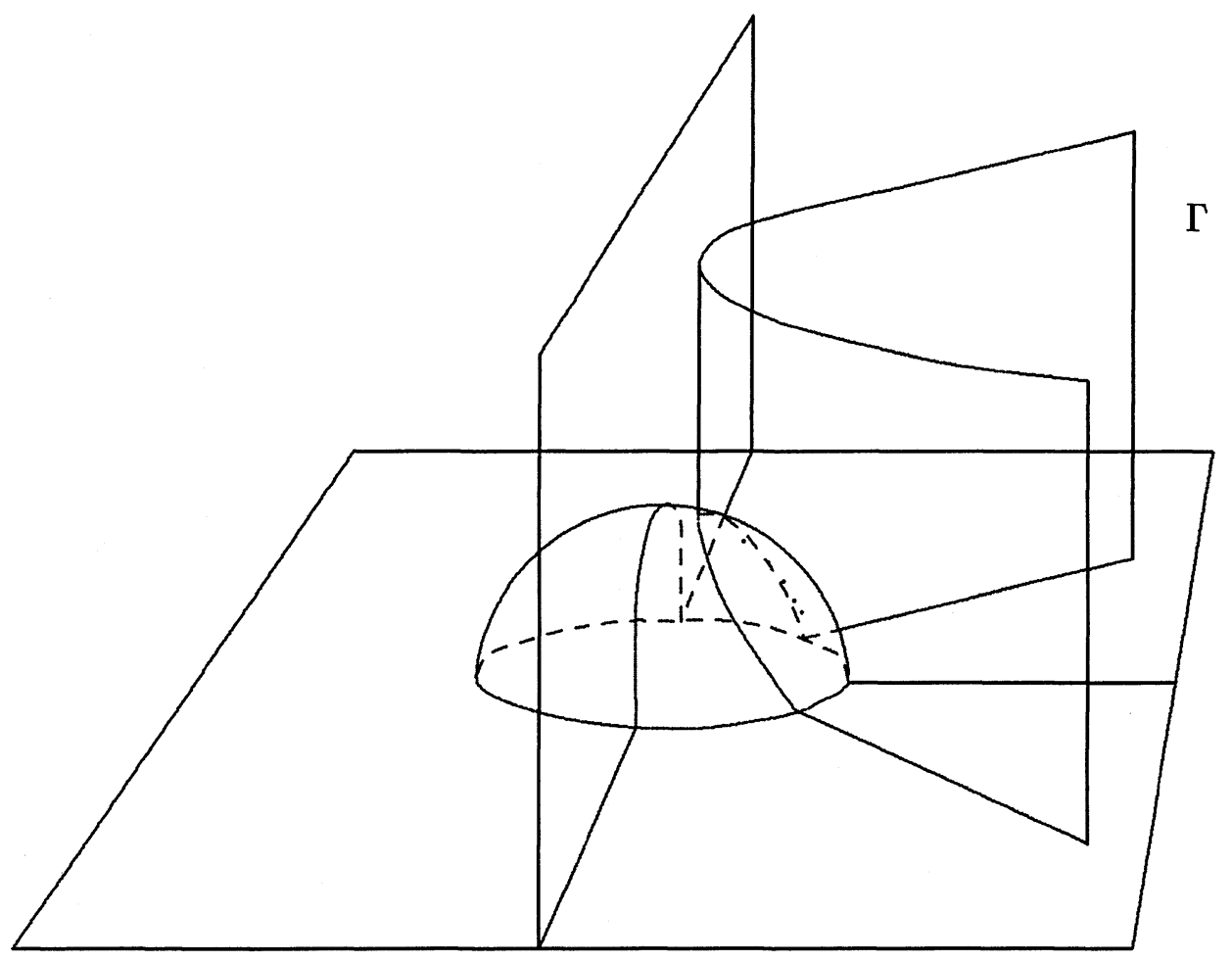

Figure 3.

\section{Lemma 8.1.}

$$
\begin{aligned}
& \Omega_{D}\left(X_{\mathrm{LHs}}^{2,[C, \infty]}\right) \equiv \pi_{H}^{*} \Omega_{D}\left(X_{\mathrm{Ls}}^{2}\right) \otimes\left|\frac{d \tau}{\tau}\right| \\
& \Omega_{D}\left(X_{\mathrm{LsHR}}^{2,[C, \infty]}\right) \equiv \pi_{\mathrm{res}}^{*} \Omega_{D}\left(X_{\mathrm{LsR}}^{2}\right) \otimes\left|\frac{d \tau}{\tau}\right| \\
& \Omega_{D}\left(X_{\mathrm{LsHR}}^{2,[C, \infty]}\right) \equiv \pi_{\mathrm{heat}}^{*} \Omega_{D}\left(X_{\mathrm{LHs}}^{2,[C, \infty]}\right) \otimes\left|\frac{d \lambda d \bar{\lambda}}{\lambda \bar{\lambda}+(\text { ias } \epsilon)^{2}}\right| .
\end{aligned}
$$

Proof. The first two follow easily from the fact that the b-density bundle lifts to the b-density bundle when a boundary face is blown up. The third equation follows from Lemma 7.1. 


\subsection{Contour spaces.}

The contour of integration, $\Gamma$, with which we perform the integral (8.1) is a p-submanifold of $X_{\mathrm{LsR}}^{0}$ of codimension one as illustrated in Figure 3. It should have the following properties:

(i) $\Gamma$ lies in the right half plane;

(ii) For $|z|>1$,

$$
\Gamma=\text { closure }\left\{\arg z= \pm \frac{\pi}{6}\right\}
$$

(iii) For $|z|<2, \Gamma$ is given by a relation $\{\operatorname{Re} z=f(\operatorname{Im} z)\}$, with $f$ smooth, such that for $|z|>1$ this relation is given by (ii) above and $f(0)=a$, where $a>0$ is real and $a^{2}$ is strictly less than the smallest nonzero eigenvalue of $\mathrm{RN}(\Delta)$;

(iv) $\Gamma$ is disjoint from the poles of $\left(\Delta-\lambda^{2}\right)^{-1}$, for ilg $\epsilon$ small.

Condition (iv) is possible and compatible with (iii) by the results of section 6 .

It follows that $\Gamma$ 'encloses' all of the spectrum of $\Delta$ except that corresponding to $L^{2}$ null space of $\Delta_{\bar{M}}$ and $z_{0} \in \operatorname{spec} \mathrm{RN}(\Delta)$, if $z_{0}=0$. We denote $\Gamma \cap B^{0}$ by $\mathcal{C}$, a contour in the right half $z$-plane enclosing all positive spectrum of $\mathrm{RN}(\Delta)$, and $\Gamma \cap B^{\mathbb{C}}$ by $\gamma_{+} \cup \gamma_{-}$, where $\gamma_{ \pm}=\{\arg \lambda= \pm \pi / 6\}$.

Since $\Gamma$ is an interior p-submanifold of $X_{\mathrm{LsR}}^{0}$ of codimension one, the inverse images of $\Gamma$ in $X_{\mathrm{LsR}}^{2}$ and $X_{\mathrm{LsHR}}^{2,[C, \infty]}$ are also interior p-submanifolds of codimension one, which we will denote $X_{\mathrm{LsC}}^{2}$ and $X_{\mathrm{LsHC}}^{2}$ respectively, the 'double contour space' and the 'double heat-contour space'. Moreover the maps $X_{\mathrm{LsHC}}^{2} \rightarrow X_{\mathrm{LsC}}^{2} \rightarrow \Gamma$ are simple b-fibrations. Since these spaces are interior p-submanifolds of the respective resolvent space, their boundary hypersurfaces are given by their intersection with boundary hypersurfaces of the ambient space. We will use the same notation for maps between the resolvent spaces and their restrictions to the contour spaces. The map $X_{\mathrm{LsHC}}^{2} \rightarrow \Gamma$ will be denoted $\pi_{\mathrm{par}}$. We also use the same notation for the boundary hypersurface of the contour spaces as for the corresponding resolvent space, and assign the same degree. With these degrees,

$$
\left|\frac{d \text { ias } \epsilon}{(\operatorname{ias} \epsilon)^{2}} \frac{d \lambda}{\lambda}\right|
$$

is a nonzero smooth section of $\Omega_{D}(\Gamma)$. Similarly, we have

$$
\begin{gathered}
\Omega_{D}\left(X_{\mathrm{LsC}}^{2}\right) \cong \pi^{*} \Omega_{D}\left(X_{\mathrm{Ls}}^{2}\right) \otimes\left|\frac{d \lambda}{\lambda}\right| \\
\Omega_{D}\left(X_{\mathrm{LsHC}}^{2}\right) \cong \pi^{*} \Omega_{D}\left(X_{\mathrm{LHs}}^{2,[C, \infty]}\right) \otimes\left|\frac{d \lambda}{\lambda}\right|
\end{gathered}
$$


It will be convenient in the proof of Proposition 8.2 to have the densities written in this product form.

We need one more space before carrying out the integral (8.1). This is a space to carry the function $e^{-t \lambda^{2}}$. Note that this is smooth, everywhere away from $\left\{|\lambda|=\infty, \operatorname{Re} \lambda^{2}<0\right\}$, on the space

$$
\left[[T, \infty]_{\tau} \times \overline{\mathbb{C}}_{\lambda} ;\{\tau=\infty, \lambda=0\}\right] .
$$

Hence by Lemma 2.1, this lifts to a smooth function on the space

$$
\begin{array}{r}
\tilde{X}_{\mathrm{LsHR}}^{0} \\
=\left[X_{\mathrm{Ls}}^{0} \times[T, \infty]_{\tau} \times \overline{\mathbb{C}}_{\lambda} ;\{\operatorname{ilg} \epsilon=0, \tau=\infty, \lambda=0\} ;\{\tau=\infty, \lambda=0\}\right. \\
\{\operatorname{ilg} \epsilon=0, \lambda=0\} ;\{\operatorname{ilg} \epsilon=0, \tau=\infty\}] \\
=\left[X_{\mathrm{Ls}}^{0} \times[T, \infty]_{\tau} \times \overline{\mathbb{C}}_{\lambda} ;\{\operatorname{ilg} \epsilon=0, \tau=\infty\} ;\{\operatorname{ilg} \epsilon=0, \tau=\infty, \lambda=0\} ;\right. \\
\\
\quad\{\operatorname{ilg} \epsilon=0, \lambda=0\} ;\{\tau=\infty, \lambda=0\}] .
\end{array}
$$

Indeed, by Lemmas 2.1, 2.5 and 2.7, this space maps down to both the space (8.4) and $X_{\mathrm{LsR}}^{0}$ with b-fibrations. Lifting $\Gamma \subset X_{\mathrm{LsR}}^{0}$ to $\tilde{X}_{\mathrm{LsHR}}^{0}$, we obtain the 'zero-heat-contour space' $X_{\mathrm{LsHC}}^{0}=\pi^{-1} \Gamma$. This is an appropriate space to carry the function $e^{-t \lambda^{2}}$, which is now smooth everywhere since $\Gamma \subset\{\operatorname{Re} \lambda \geq 0\}$. Now observe that, in fact, $\pi^{-1} \Gamma$ does not meet the face $\{\tau=\infty, \lambda=0\}$, the last face to be blown up above, and so for the purpose of defining $X_{\mathrm{LsHC}}^{0}$ this blowup could be omitted. Let us define the 'zeroheat-resolvent space' $X_{\mathrm{LsHR}}^{0}$ (without tilde) to be the space in (8.5) with this blowup omitted:

$$
\begin{array}{r}
X_{\mathrm{LsHR}}^{0}=\left[X_{\mathrm{Ls}}^{0} \times[C, \infty]_{\tau} \times \overline{\mathbb{C}} ;\{\operatorname{ilg} \epsilon=0, \tau=\infty\} ;\{\operatorname{ilg} \epsilon=0, \tau=\infty, \lambda=0\} ;\right. \\
\{\operatorname{ilg} \epsilon=0, \lambda=0\}]
\end{array}
$$

Then, by Lemma 2.5, and using the second description (8.3) of $X_{\mathrm{LsHR}}^{2,[C, \infty]}$, the map

$$
X_{\mathrm{Ls}}^{2} \times[C, \infty]_{\tau} \times \overline{\mathbb{C}} \longrightarrow X_{\mathrm{Ls}}^{0} \times[C, \infty]_{\tau} \times \overline{\mathbb{C}}
$$

lifts to a b-fibration

$$
X_{\mathrm{LSHR}}^{2,[C, \infty]} \longrightarrow X_{\mathrm{LsHR}}^{0}
$$

and so

$$
X_{\mathrm{LsHC}}^{2} \longrightarrow X_{\mathrm{LsHC}}^{0}
$$

is also a b-fibration. The point of this discussion of $\tilde{X}_{\mathrm{LsHR}}^{0}$ and $X_{\mathrm{LsHR}}^{0}$ is to show that $e^{-t \lambda^{2}}$ lifts to be smooth on $X_{\mathrm{LsHC}}^{2}$, although it is not smooth on 
$X_{\mathrm{LSHR}}^{2,[C, \infty]}$. Finally we have the bundle isomorphism which we need to perform the integral (8.1):

$$
\Omega_{D}\left(X_{\mathrm{LsHC}}^{2}\right) \cong \Omega_{D}^{\frac{1}{2}}\left(X_{\mathrm{LsR}}^{2}\right) \otimes \Omega_{D}^{\frac{1}{2}}\left(X_{\mathrm{LHs}}^{2,[C, \infty]}\right) \otimes \Omega_{D}^{\frac{1}{2}}\left(X_{\mathrm{LsHC}}^{0}\right) \otimes\left|\frac{d(\operatorname{ilg} \epsilon)}{(\mathrm{ilg} \epsilon)^{2}}\right|^{-\frac{1}{2}}
$$

\subsection{Behaviour as $t \rightarrow \infty$.}

The contour $\Gamma$ does not enclose the eigenvalues of $\Delta_{\epsilon}$ corresponding to $L^{2}$ zero modes of $\Delta_{\bar{M}}$ or the value $0 \in \operatorname{spec} \mathrm{RN}(\Delta)$. Hence, the integral (8.1) will yield the heat kernel projected off these modes. We will denote this by $e^{-t \Delta_{\epsilon}^{\perp}}$. Similarly, $\mathrm{RN}(\Delta)^{\perp}$ and $\Delta \frac{\perp}{M}$ denote these operators projected off their $L^{2}$ zero modes. Also let $\Pi_{0}$ denote projection onto the 0 eigenvalue of $\mathrm{RN}(\Delta)$. In the previous subsection, spaces and maps were constructed as follows:

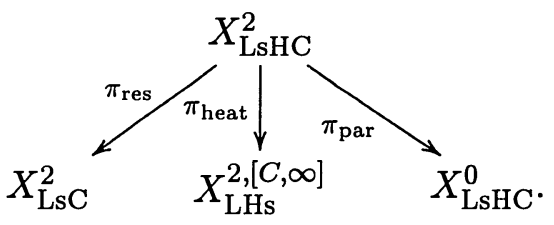

Explicitly, including density factors, $e^{-t \Delta_{\epsilon}^{\perp}}$ is given by

$$
\begin{aligned}
& e^{-t \Delta_{\epsilon}^{\perp}} \cdot \kappa^{\frac{1}{2}} \\
= & \pi_{\text {heat } *}\left(\pi_{\text {res }}^{*}\left(\Delta_{\epsilon}-\lambda^{2}\right)^{-1} \cdot \pi_{\text {heat }}^{*} \kappa^{\frac{1}{2}} \cdot \pi_{\text {par }}^{*}\left(2 \lambda^{2} e^{-t \lambda^{2}} \omega^{\frac{1}{2}}\right) \cdot\left|\frac{d(\operatorname{ilg} \epsilon)}{(\operatorname{ilg} \epsilon)^{2}}\right|^{-\frac{1}{2}}\right)
\end{aligned}
$$

where $\kappa$ is the canonical density on $X_{\mathrm{LHs}}^{2,[C, \infty]}$, and $\omega$ is the $D$-density $\mid \frac{d t}{t} \frac{d \operatorname{ilg} \epsilon}{\operatorname{lig} \epsilon^{2}}$

$\frac{d \lambda}{\lambda} \mid$ on $X_{\mathrm{LsHC}}^{0}$. The ' $2 \lambda^{2}$ ' arises because the measure in (8.1) is $2 \lambda d \lambda$ and there is are formal factors of $\lambda^{-1 / 2}$ in both the resolvent and in $\omega^{1 / 2}$ to be cancelled. Since $\left(\Delta_{\epsilon}-\lambda^{2}\right)^{-1} \in \lambda^{-2} \Psi_{\mathrm{LsR}}^{-2,0}(X)$, the product in (8.7) is a smooth $D$-density on $X_{\text {LsHC }}^{2}$. Note that $\pi_{\text {heat }}$ has the property that every boundary hypersurface in $X_{\mathrm{LsHC}}^{2}$ maps to a boundary hypersurface of the same degree, and each boundary hypersurface of $X_{\mathrm{LHs}}^{2,[C, \infty]}$ is the image of exactly two boundary hypersurfaces of $X_{\mathrm{LsHC}}^{2}$, which intersect. Hence, by the pushforward theorem,

$$
e^{-t \Delta_{\epsilon}^{\perp}} \in \mathcal{A}_{\mathrm{phg}}^{0 \overline{\mathrm{U} 0}}\left(X_{\mathrm{LHs}}^{2,[C, \infty]} ; \Omega_{D}\right),
$$


where $0 \overline{\mathrm{U} 0} 0$ is the natural index family which assigns to each boundary hypersurface the index set

$$
\{(n, 0),(n, 1) ; n \in \mathbb{N}\}
$$

To calculate the coefficients of these terms, we shall use Lemma 2.4. Note that the index set allows logarithmic terms at every boundary hypersurface. We show below that in fact the logarithmic terms do not appear. If $e^{-t \Delta_{\epsilon}^{\perp}}$ is in $\rho_{G}^{k} C^{\infty}\left(X_{\mathrm{LHs}}^{2,[C, \infty]} ; \Omega_{D}^{1 / 2}\right)$ at a boundary hypersurface $G$ write the restriction of (ias $\epsilon)^{-k} e^{-t \Delta_{\epsilon}^{\perp}} \mid G$ as $H^{\perp(k)}(G)$. The following proposition contains the 'long time information' of Theorem 1.2 and more:

Proposition 8.2. The heat kernel projected off null modes, $e^{-t \Delta_{\epsilon}^{\perp}}$, is a smooth $D$-density on the long-time heat space $X_{\mathrm{LHs}}^{2,[C, \infty]}$. The top models at each face are given by:

$$
\begin{aligned}
H^{\perp(1)}\left(B_{m n}^{\infty}\right) & =\mathfrak{T}\left(e^{-T \mathrm{RN}(\Delta)^{\perp}}\left\lceil B_{m n}^{\infty}\right)\right. \\
H^{\perp(j)}\left(B_{t \infty}\right) & =\emptyset \text { for all } j \\
H^{\perp(0)}\left(B_{00}\right) & =e^{-t \Delta \frac{\perp}{M}} \\
H^{\perp(0)}\left(B_{11}\right) & =e^{-t \Delta_{\bar{H}}} \\
H^{\perp(0)}\left(B_{33}\right) & =e^{-t \Delta_{\bar{H}}} \text { and } \\
H^{\perp(1)}\left(B_{m n}\right) & =\mathfrak{T}\left(-\Pi_{0}\left\lceil B_{m n}\right)\right. \text { otherwise. }
\end{aligned}
$$

Proof. First consider the faces $B_{m n}^{\infty}$. The boundary hypersurfaces in $X_{\mathrm{LsHC}}^{2}$ that map to $B_{m n}^{\infty}$ are $B_{m n}^{\infty, 0}$ and $B_{m n}^{\infty, \mathbb{C}}$. At $B_{m n}^{\infty, \mathbb{C}}$ the function $e^{-t \lambda^{2}}$, lifted to $X_{\mathrm{LsHC}}^{2}$, vanishes to infinite order so there is no contribution to the asymptotic expansion at $B_{m n}^{\infty}$ from $B_{m n}^{\infty, C}$, and moreover, (2.15) shows that there are no logarithmic terms at $B_{m n}^{\infty}$.

At $B_{00}^{\infty, 0}$ the top term in the integrand (8.7) is

$$
\begin{aligned}
& \lambda^{-2} \operatorname{proj}_{L^{2}}\left|d \nu d \nu^{\prime} \frac{d t}{t}\right| e^{-T z^{2}} 2 \lambda d \lambda \\
= & z^{-2} \operatorname{proj}_{L^{2}}\left|d \nu d \nu^{\prime} \frac{d t}{t}\right| e^{-T z^{2}} 2 z d z .
\end{aligned}
$$

This is integrated over a contour which encloses no poles of the integrand, and which vanishes rapidly as $z \rightarrow \infty$. Hence, this integral gives zero; there is no contribution to the face $B_{00}^{\infty}$ of order (ias $\left.\epsilon\right)^{0}$. 
The top order contribution from each other face $B_{m n}^{\infty, 0}$, is

$$
\begin{aligned}
H^{\perp(1)}\left(B_{m n}^{\infty}\right) & =(\operatorname{ias} \epsilon)^{-1} \int_{\mathcal{C}} e^{-t \lambda^{2}} \frac{\mathfrak{T}\left(K_{m n}^{(-1)}\right)}{\operatorname{ias} \epsilon} 2 \lambda d \lambda\left|d \nu d \nu^{\prime} \frac{d \operatorname{ias} \epsilon}{(\operatorname{ias} \epsilon)^{2}} \frac{d t}{t}\right| \\
& =\int_{\mathcal{C}} e^{-T z^{2}} \mathfrak{T}\left(K_{m n}^{(-1)}\right) 2 z d z\left|d \nu d \nu^{\prime} \frac{d \operatorname{ias} \epsilon}{(\operatorname{ias} \epsilon)^{2}} \frac{d t}{t}\right| \\
& =\mathfrak{T}\left(e^{T \operatorname{RN}(\Delta)^{\perp}} \mid B_{m n}^{\infty}\right),
\end{aligned}
$$

since the integral encloses all points of the spectrum of $\mathrm{RN}(\Delta)$ except 0 .

At $B_{t \infty}, e^{-t \lambda^{2}}$ vanishes to infinite order so $e^{-t \Delta_{\epsilon}^{\perp}} \equiv 0$ to all orders at $B_{t \infty}$.

Next consider the faces $B_{m n}$. The boundary hypersurfaces that map to $B_{m n}$ are $B_{m n}^{0}$ and $B_{m n}^{\mathbb{C}}$. Recall that, by Theorem 7.2, the resolvent has just one term in the full Taylor series at $B_{00}^{\mathbb{C}}, B_{11}^{\mathbb{C}}, B_{33}^{\mathbb{C}}$, namely $\left(\Delta_{\bar{M}}-\lambda^{2}\right)^{-1}$, $\left(\Delta_{\bar{H}}-\lambda^{2}\right)^{-1}$ and $\left(\Delta_{\bar{H}}-\lambda^{2}\right)^{-1}$ respectively, and vanishes to infinite order at the other faces $B_{m n}^{\mathbb{C}}$. At the faces $B_{m n}^{0}$, the top terms are, for $B_{00}^{0}, G_{00}^{(-2)}=$ $z^{-2} \operatorname{proj}_{L^{2}}$, and for the other faces, $G_{m n}^{(-1)}=\mathfrak{T}\left(K_{m n}^{(-1)}\right)$. The integrand in (8.7) also contains the factor $\lambda^{2}$, which increases the power at which the terms on the faces $B_{m n}^{0}$ appear by two. Therefore, by (2.15), the only possibility for a logarithmic term is at $B_{00}\left(X_{\mathrm{LHs}}^{2,[C, \infty]}\right)$, at order zero. This term comes from the corner $B_{00}^{0} \cap B_{00}^{\mathbb{C}}$, which consists of two copies of $B_{00}\left(X_{\mathrm{LHs}}^{2,[C, \infty]}\right)$. The integrand is, at this corner, equal to $2 \operatorname{proj}_{L^{2}} \cdot \mid d \nu d \nu^{\prime} d \operatorname{ilg} \epsilon /$ ilg $\epsilon d t / t \mid$. Note that the direction of integration is opposite on the two copies: at one the contour is coming in from infinity, and at the other the contour is going out to infinity. Since the contour integral is a directed integral, these two contributions cancel, giving no logarithmic term on $X_{\mathrm{LHs}}^{2,[C, \infty]}$.

The smooth term at $B_{00}$ is, by Lemma 2.4, given by the sum of the b-integrals on $B_{00}^{0}$ and $B_{00}^{\mathbb{C}}$. The regularized integral on $B_{00}^{\mathbb{C}}$ is

$$
\lim _{\delta \downarrow 0}\left[\sum_{ \pm} \int_{\gamma_{ \pm} \cap|\lambda \geq \delta|}\left(\Delta-\lambda^{2}\right)^{-1} e^{-t \lambda^{2}} 2 \lambda^{2} \frac{d \lambda}{\lambda} \pm \log \delta \operatorname{proj}_{L^{2}}\right] .
$$

The $\log \delta$ term is zero after summing over \pm , as also follows from the vanishing of the term coming from the corner. Expanding the integrand,

$$
\left(\left(\Delta-\lambda^{2}\right)^{-1} e^{-t \lambda^{2}} 2 \lambda^{2} \frac{d \lambda}{\lambda}\right)=\operatorname{proj}_{L^{2}}\left(\frac{d \lambda}{\lambda} 2 e^{-t \lambda^{2}}\right)+\lambda\left(\Delta \frac{\perp}{M}-\lambda^{2}\right)^{-1} 2 e^{-t \lambda^{2}} \alpha_{1}^{\prime} \lambda
$$

the second term is holomorphic near zero, and so is absolutely integrable. Letting $\delta \rightarrow 0$ we obtain $e^{-t \Delta \frac{1}{M}}$ from the integral. For the first term we may 
write

$$
\begin{array}{r}
\operatorname{proj}_{L^{2}} \int_{\gamma_{ \pm} \cap|\lambda \geq \delta|} 2 e^{-t \lambda^{2}} \frac{d \lambda}{\lambda}= \\
\int_{\delta e^{ \pm i \pi / 6}}^{1} 2 e^{-t \lambda^{2}} \operatorname{proj}_{L^{2}} \frac{d \lambda}{\lambda} \mp \int_{1}^{\infty e^{ \pm i \pi / 6}} 2 e^{-t \lambda^{2}} \operatorname{proj}_{L^{2}} \frac{d \lambda}{\lambda} \text { and so } \\
\lim _{\delta \downarrow 0} \sum_{ \pm} \operatorname{proj}_{L^{2}} \int_{\gamma_{ \pm} \cap|\lambda \geq \delta|} 2 e^{-t \lambda^{2}} \frac{d \lambda}{\lambda}=\lim _{\delta \downarrow} \sum_{ \pm} \int_{\delta e^{ \pm i \pi / 6}}^{1} 2 \operatorname{proj}_{L^{2}} \frac{d \lambda}{\lambda} .
\end{array}
$$

Now consider $B_{00}^{0}$. This regularized integral is

$$
\lim _{\delta \downarrow 0} 2 \operatorname{proj}_{L^{2}}\left[\int_{\mathcal{C} \cap|\lambda \leq 1 / \delta|} \frac{d z}{z}\right]
$$

By changing variable of integration from $z$ to $1 / z$ in this integral, one finds that the sum of this term and (8.10) is zero. Therefore the total contribution to $H^{\perp(0)}\left(B_{00}\right)$ is $e^{-t \Delta \frac{\perp}{M}}$.

The top term at $B_{11}$ and $B_{33}$ is given by

$$
H^{\perp(0)}\left(B_{11}\right)=H^{\perp(0)}\left(B_{33}\right)=\int_{\gamma_{ \pm}}\left(\Delta-\lambda^{2}\right)^{-1} e^{-t \lambda^{2}} 2 \lambda^{2} \frac{d \lambda}{\lambda}=e^{-t \Delta_{\bar{H}}}
$$

Finally, let us calculate the $H^{\perp(1)}$ term at each face $B_{m n}$, for $(m n) \neq$ (00), (11), (33). This comes from the top term at $B_{m n}^{0}$ :

$$
H^{\perp(1)}\left(B_{m n}\right)=\int_{\mathcal{C}} \mathfrak{T}\left(\left(\mathrm{RN}(\Delta)-z^{2}\right)^{-1}\right) 2 z d z .
$$

From (5.9) it follows that on these faces $\left(\mathrm{RN}(\Delta)-z^{2}\right)^{-1}$ is exponentially decreasing as $|z| \rightarrow \infty, \arg z= \pm \pi / 6$. Hence, by dominated convergence we can calculate this integral as

$$
\begin{aligned}
H^{\perp(1)}\left(B_{m n}\right) & =\lim _{T \downarrow 0} \int_{\mathcal{C}} e^{-T z^{2}} \mathfrak{T}\left(\left(\operatorname{RN}(\Delta)-z^{2}\right)^{-1} 2 z d z\right) \\
& =\lim _{T \downarrow 0} \mathfrak{T}\left(e^{-T \operatorname{RN}(\Delta)}-\Pi_{0}\right) \\
& =-\mathfrak{T}\left(\Pi_{0}\right)
\end{aligned}
$$


on these faces. This completes the proof of the proposition.

\subsection{Very small eigenvalues.}

In the last subsection we split off from $\Delta_{\epsilon}$ the projection onto eigenfunctions corresponding to very small eigenvalues. We will denote by $\Pi_{\epsilon}$ the projection onto these eigenfunctions, and write $\Pi_{L^{2}}$ (respectively $\Pi_{0}$ ) for the projection onto eigenvalues corresponding to $L^{2}$ zero modes (resp zero modes of $\mathrm{RN}(\Delta)$ ). Let us analyze the behaviour of $\Pi_{\epsilon} e^{-t \Pi_{\epsilon}}$.

Proposition 8.3. The operator $\Pi_{\epsilon} e^{-t \Pi_{\epsilon}}$ is a uniformly finite rank operator, with Schwartz kernel smooth on $X_{\mathrm{LHs}}^{2,[C, \infty]}$ everywhere except possibly up to $B_{t \infty}$. The top terms at each face $\neq B_{\mathrm{t} \infty}$ are

$$
\begin{aligned}
\left(\Pi_{\epsilon} e^{-t \Pi_{\epsilon}}\right)^{(0)}\left(B_{00}\right)= & \left(\Pi_{\epsilon} e^{-t \Pi_{\epsilon}}\right)^{(0)}\left(B_{00}^{\infty}\right)=\Pi_{L^{2}} \\
& \left(\Pi_{\epsilon} e^{-t \Pi_{\epsilon}}\right)^{(1)}\left(B_{m n}\right)=\mathfrak{T}\left(\Pi_{0}\right) \\
\left(\Pi_{\epsilon} e^{-t \Pi_{\epsilon}}\right)^{(1)}\left(B_{m n}^{\infty}\right) & =\mathfrak{T}\left(\Pi_{0}\right) .
\end{aligned}
$$

Proof. The operator $\Pi_{\epsilon} e^{-t \Pi_{\epsilon}}$ is given by a contour integral

$$
\Pi_{\epsilon} e^{-t \Pi_{\epsilon}}=\frac{i}{2 \pi} \int_{|z|=c}\left(\Delta-\lambda^{2}\right)^{-1} e^{-t \lambda^{2}} 2 \lambda d \lambda
$$

for $c$ small enough that the contour excludes all points of $\operatorname{spec} \mathrm{RN}(\Delta)$ apart from zero. By the results of section 6 , the contour encloses precisely those eigenfunctions corresponding to $\Pi_{\epsilon}$ and no others, for $\epsilon$ sufficiently small. The problem with this integral is that the function $e^{-t \lambda^{2}}$ is growing rapidly on the (appropriate) heat-contour space. To fix this, weight both sides with a factor small enough to kill this growth:

$$
e^{-t c^{2}(\mathrm{ias} \epsilon)^{2}} \cdot \Pi_{\epsilon} e^{-t \Pi_{\epsilon}}=\frac{i}{2 \pi} \int_{|z|=c}\left(\Delta-\lambda^{2}\right)^{-1} e^{-t c^{2}(\mathrm{ias} \epsilon)^{2}} e^{-t \lambda^{2}} 2 \lambda d \lambda .
$$

By the pushforward theorem this is smooth on $X_{\mathrm{LHs}}^{2,[C, \infty]}$. The added factor $e^{-t c^{2}(\text { ias } \epsilon)^{2}}$ is smooth and nonzero on all faces except $B_{\mathrm{t} \infty}$, where it vanishes rapidly. Hence $\Pi_{\epsilon} e^{-t \Pi_{\epsilon}}$ itself is smooth everywhere except possibly at $B_{\mathrm{t} \infty}$.

Notice that there is no reason to believe that $\Pi_{\epsilon} e^{-t \Pi_{\epsilon}}$ is in fact smooth up to this face, since in principle the small eigenvalues of $\supset_{\epsilon}$ could cross zero 
infinitely often as $\epsilon \rightarrow 0$.

\subsection{Full Heat Kernel.}

To obtain the full heat kernel we need to discuss the heat kernel for small time $\left[0, C^{2}\right]$ and combine it with the long time heat kernel constructed above. This is a simple matter of lifting the result from Part I to our logarithmic spaces.

In Part I, the heat kernel was constructed on the space

$$
X_{\mathrm{hs}}^{2}=\left[X_{s}^{2} \times[0, \infty]_{t} ;\left(\Delta_{s} \times\{t=0\}\right),\{d t\}\right] .
$$

Here $\Delta_{s}$ is the diagonal p-submanifold in $X_{s}^{2}$ and the blowup is performed parabolically, with the extra argument, $d t$, giving the directions in which the homogeneity is two. (See [22], chapter 7). For our purpose we just need this space restricted to $\left\{t \leq C^{2}\right\}$. The heat kernel for finite time lifts to the space

$$
X_{\mathrm{LHs}}^{2,[0, C]}=\left[X_{\mathrm{Ls}}^{2} \times\left[0, C^{2}\right]_{t} ;\left(\Delta_{\mathrm{Ls}} \times\{t=0\}\right),\{d t\}\right] .
$$

In fact, since $\Delta_{s}$ is transverse to the boundary of $X_{s}^{2}$, the logarithmic and total blowups that turn $X_{s}^{2}$ into $X_{\mathrm{Ls}}^{2}$ commute with the diagonal blowup. Thus there is a blow-down map $X_{\mathrm{LHs}}^{2,[0, C]} \rightarrow X_{\mathrm{hs}}^{2} \cap\left\{t \leq C^{2}\right\}$ and we lift the heat kernel by pulling back under this map. (We must also multiply the heat kernel by $(t \epsilon)^{-1 / 2}$ to correct for different normalization of densities used in Part I.) Then the heat kernel lifts to be a $D$-half-density on $X_{\mathrm{LHs}}^{2,[0, C]}$, with the models

$$
\begin{aligned}
& H^{(0)}\left(B_{00}\right)=e^{-t \Delta_{\bar{M}}} \\
& H^{(0)}\left(B_{11}\right)=e^{-t \Delta_{\bar{H}}} \\
& H^{(0)}\left(B_{33}\right)=e^{-t \Delta_{\bar{H}}}
\end{aligned}
$$

and all other models are identically zero. The two heat spaces $X_{\mathrm{LHs}}^{2,[0, C]}$ and $X_{\mathrm{LHs}}^{2,[C, \infty]}$ are both just a product of $X_{\mathrm{Ls}}^{2}$ with a time interval (canonically) away from $t=0$ and infinity. Hence the spaces can be identified at $t=C^{2}$ to produce the full heat space $X_{\mathrm{LHs}}^{2}$. Since the heat kernel is unique, it extends smoothly across $t=C^{2}$ to be the full heat kernel of this space. Let us summarize the properties of the full heat kernel:

Theorem 8.4. The heat kernel is a $D$-density on the heat space $X_{\mathrm{Ls}}^{2}$, $t^{-n / 2} \times$ smooth near $t=0$, and smooth everywhere else except (possibly) at 
the face $B_{\mathrm{t} \infty}$. The top terms at each face $\neq B_{\mathrm{t} \infty}$ are given by:

$$
\begin{aligned}
H^{(0)}\left(B_{00}^{\infty}\right) & =\Pi_{L^{2}} \\
H^{(1)}\left(B_{m n}^{\infty}\right) & =\mathfrak{T}\left(e^{-T \operatorname{RN}(\Delta)}\right), T=t(\operatorname{ias} \epsilon)^{2} \text { for other } B_{m n}^{\infty} ; \\
H^{(0)}\left(B_{00}\right) & =e^{-t \Delta_{\bar{M}}} \\
H^{(0)}\left(B_{11}\right) & =e^{-t \Delta_{\bar{H}}} \\
H^{(0)}\left(B_{33}\right) & =e^{-t \Delta_{\bar{H}}} \text { and } \\
H^{(j)}\left(B_{m n}\right) & =0 \text { for other } B_{m n} .
\end{aligned}
$$

\section{Limit of Eta Invariant.}

\subsection{Eta invariant.}

The eta invariant of $\partial_{\epsilon}$ is given for each $\epsilon>0$ by the formula (1.1):

$$
\eta\left(\widetilde{\partial}_{\epsilon}\right)=\frac{1}{\sqrt{\pi}} \int_{0}^{\infty} t^{\frac{1}{2}} \operatorname{Tr}\left(\widetilde{\partial}_{\epsilon} e^{-t \widetilde{\partial}_{\epsilon}^{2}}\right) \frac{d t}{t} .
$$

At $\epsilon=0$, the b-eta invariant is defined by

$$
\eta_{b}\left(\check{\partial}_{M_{ \pm}}\right)=\frac{1}{\sqrt{\pi}} \int_{0}^{\infty} t^{\frac{1}{2}} b-\operatorname{Tr}\left(\check{\partial}_{M_{ \pm}} e^{-t \widetilde{\widetilde{J}}_{M_{ \pm}}^{2}}\right) \frac{d t}{t},
$$

where b-Tr is defined in section 2.3. We will show below that this is independent of the choice of boundary defining function in the definition of b-Tr.

As in the previous section we split the operator $\partial_{\epsilon}$ into two pieces, $\partial_{\epsilon}^{\perp}$, orthogonal to the finite number of eigenfunctions corresponding to the $L^{2}$ null space of $\partial_{0}$ and $0 \in \operatorname{spec} R N(\widetilde{\partial})$, and the projection $\Pi_{\epsilon}$ onto these eigenfunctions. Then

$$
\eta\left(\widetilde{\partial}_{\epsilon}\right)=\eta\left(\widetilde{\partial}_{\epsilon}^{\perp}\right)+\eta\left(\Pi_{\epsilon}\right)=\eta\left(\partial_{\epsilon}^{\perp}\right)+\eta_{\mathrm{fd}}(\epsilon)
$$

where $\eta_{\mathrm{fd}}(\epsilon)$ is just the signature of $\Pi_{\epsilon}$, that is, the dimension of the space of eigenfunctions with positive eigenvalue minus the dimension of the space of eigenfunctions with negative eigenvalue. Hence $\eta_{\mathrm{fd}}$ takes values in $\{0, \pm 1, \ldots$,

$\pm N\}$, where $N$ is the rank of $\Pi_{\epsilon}$. The $\partial^{\perp}$ part of (9.3) we calculate by applying the Pushforward theorem to the integral (9.1), replacing $\varpi_{\epsilon}$ with $\delta_{\epsilon}^{\perp}$. As 
this involves the trace of $\widetilde{\partial}_{\epsilon} e^{-t \widetilde{\partial}_{\epsilon}^{2}}$ we first consider the diagonal submanifold of $X_{\mathrm{LHs}}^{2}$.

\subsection{The diagonal of the Logarithmic heat space.}

The diagonal $\Delta_{\mathrm{LHs}}$ of $X_{\mathrm{LHs}}^{2}$ is the lift of $\Delta_{\mathrm{Ls}} \times[0, \infty]_{\tau} \subset X_{\mathrm{Ls}}^{2}$ to $X_{\mathrm{LHs}}^{2}$. It is transversal to all boundaries and has the form

$$
\begin{array}{r}
\Delta_{\mathrm{LHs}}=\left[\Delta_{\mathrm{Ls}} \times[0, \infty]_{\tau} ;\left(B_{33} \cap \Delta_{\mathrm{Ls}}\right) \times\{\tau=\infty\} ;\left(B_{00} \cap \Delta_{\mathrm{Ls}}\right) \times\{\tau=\infty\} ;\right. \\
\left.\left(B_{11} \cap \Delta_{\mathrm{Ls}}\right) \times\{\tau=\infty\}\right] .
\end{array}
$$

We define the degrees of boundary hypersurfaces of $\Delta_{\mathrm{LHs}}$ to be those of the corresponding hypersurfaces of $X_{\mathrm{LHs}}^{2}$. On a compact manifold without boundary, $N$, one has the canonical density bundle isomorphism

$$
\Omega^{\frac{1}{2}}(N \times N) \uparrow \Delta \equiv \Omega(\Delta) .
$$

This means that, given a half-density on $N \times N$ representing the Schwartz kernel of a (suitable) operator, one can restrict to the diagonal and integrate the resulting density to obtain the trace. Here, our version of this isomorphism is, from (4.16),

$$
\left.\Omega_{D}^{\frac{1}{2}}\left(X_{\mathrm{LHs}}^{2}\right)\left|\Delta_{\mathrm{LHs}} \otimes\right| \frac{d(\mathrm{ilg} \epsilon)}{(\mathrm{ilg} \epsilon)^{2}}\right|^{\frac{1}{2}} \equiv \Omega_{D}\left(\Delta_{\mathrm{LHs}}\right) .
$$

Hence, if $\iota$ is the inclusion $\Delta_{\mathrm{LHs}} \hookrightarrow X_{\mathrm{LHs}}^{2}$ and $p$ is the map $X_{\mathrm{LHs}}^{2} \rightarrow$ $\left[0, \text { ilg } \epsilon_{0}\right]_{i l g} \epsilon$, we can express the eta invariant for $\widetilde{\partial}_{\epsilon}^{\perp}$ as

$$
\eta\left(\partial_{\epsilon}^{\perp}\right)=\frac{1}{\sqrt{\pi}} p_{*}\left(t^{\frac{1}{2}} \cdot \iota^{*}\left(\operatorname{tr} \partial_{\epsilon}^{\perp} e^{-t \delta_{\epsilon}^{\perp 2}}\right) \cdot\left|\frac{d(\operatorname{ilg} \epsilon)}{(\operatorname{ilg} \epsilon)^{2}}\right|^{\frac{1}{2}}\right) \cdot\left|\frac{d(\mathrm{ilg} \epsilon)}{(\mathrm{ilg} \epsilon)^{2}}\right|^{-1} .
$$

\subsection{Asymptotic expansion of $\eta$ as $\epsilon \rightarrow 0$.}

Consider the integral (9.4). To apply the Pushforward Theorem (Theorem 2.3), we must check the integrability condition $\mathcal{K}(G)>d(G)$ for all $G$ such that $p(G)=\left[0\right.$, ilg $\left.\epsilon_{0}\right]$, that is, for $G=B_{\mathrm{tf}}, B_{\mathrm{t} \infty}$. Proposition 8.2 asserts that the integrand is rapidly vanishing at $B_{\mathrm{t} \infty}$. At $B_{\mathrm{tf}}$ it is well known that the heat kernel has growth of order $t^{-n / 2}$. However after taking the pointwise trace, Patodi in [27] showed that extensive cancellations occur in 
the integrand and the growth is of order $t^{1 / 2}$, making (9.4) integrable. This also follows rather simply using Getzler's rescaling (see [13]).

The structure of the integrand on the double heat space follows readily from Theorem 8.2.

Proposition 9.1. On $X_{\mathrm{LHs}}^{2}$, the integrand $I$ in $(9.4)$ is in $C^{\infty}\left(X_{\mathrm{LHs}}^{2} ; \Omega_{D}^{\frac{1}{2}}\right)$ and the leading terms at each diagonal face at ias $\epsilon=0$ are

$$
\begin{aligned}
& I^{(1)}\left(B_{m n}^{\infty}\right)=\frac{1}{\sqrt{\pi}} \mathfrak{T}\left(T^{\frac{1}{2}} \operatorname{tr} \operatorname{RN}\left(\check{\partial}_{\epsilon}\right) e^{-T \operatorname{RN}\left(\varpi_{\epsilon}\right)^{2}}\right) \\
& I^{(0)}\left(B_{00}\right)=\frac{1}{\sqrt{\pi}} t^{\frac{1}{2}} \operatorname{tr} \delta_{\bar{M}} e^{-t \bar{\partial}_{\bar{M}}^{2}} \\
& I^{(0)}\left(B_{11}\right)=\frac{1}{\sqrt{\pi}} t^{\frac{1}{2}} \operatorname{tr} \varlimsup_{\bar{H}} e^{-t \widetilde{\partial}_{\bar{H}}^{2}} \\
& I^{(0)}\left(B_{33}\right)=\frac{1}{\sqrt{\pi}} t^{\frac{1}{2}} \operatorname{tr} \varlimsup_{\bar{H}} e^{-t \widetilde{\partial}_{\bar{H}}^{2}} .
\end{aligned}
$$

Proof. The last three lines are immediate from Theorem 8.2. The first line follows for $B_{22}^{\infty}$ since there $ð=\check{\partial}_{H}+($ ias $\epsilon) \operatorname{RN}(ð)+O\left((\text { ias } \epsilon)^{2}\right)$ and $t^{1 / 2}=$ (ias $\epsilon)^{-1} T^{1 / 2}$. This determines the other top terms on $B_{m n}^{\infty}$ by compatibility.

Of the six boundary faces at ias $\epsilon=0$, four, $B_{00}, B_{11}, B_{00}^{\infty}$ and $B_{11}^{\infty}$ have degree one and the other two, $B_{33}, B_{33}^{\infty}$ have degree two. Using the Pushforward theorem and the result above, we see that for small $\epsilon$

$$
\eta\left(\widetilde{\partial}_{\epsilon}\right) \in \mathcal{A}_{\text {phg }}^{\mathcal{E}}\left(\left[0, \mathrm{ilg} \epsilon_{0}\right]_{\text {ilg } \epsilon}\right),
$$

where $\mathcal{E}$ is the index set

$$
\mathcal{E}=\{(-1,0)\} \cup\{(n, 0),(n, 1) ; n \in \mathbb{N}\},
$$

that is, asymptotically

$$
\eta\left(\check{\partial}_{\epsilon}\right) \sim \sum_{(n, k) \in \mathcal{E}} a_{n, k}(\operatorname{ias} \epsilon)^{n}(\log (\operatorname{ias} \epsilon))^{k} .
$$

There are exactly two terms in this expansion that could prevent $\eta\left(\widetilde{\partial}_{\epsilon}\right)$ from having a limit as $\epsilon \rightarrow 0, a_{-1,0}$ and $a_{0,1}$. The $(-1,0)$ term comes from $B_{33}$ and the $(0,1)$ term comes from $B_{11} \cap B_{33}^{\infty}$ and $B_{00} \cap B_{33}^{\infty}$. Let us compute 
these coefficients. The term $a_{-1,0}$ is the integral of $I^{(0)}\left(B_{33}\right)$. Consider the pointwise trace

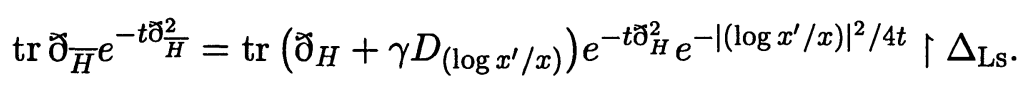

Note that $e^{-t \delta_{H}^{2}}$ is diagonal with respect to the grading of the spinor bundle $S=S^{+} \oplus S^{-}$, while $\partial_{\bar{H}}$ is the sum of a piece which is off diagonal with respect to the grading of $S$ and a piece which kills $e^{-\left|\left(\log x^{\prime} / x\right)\right|^{2} / 4 t}$ at $\Delta_{\mathrm{Ls}}=$ $\left\{\left(\log x^{\prime} / x\right)=0\right\}$. Hence this term is identically zero, and therefore $a_{-1,0}=0$. This also proves the remark above that (9.2) is independent of the choice of boundary defining function. In fact, after taking the pointwise trace, the integral is absolutely convergent.

Next, $a_{0,1}$ is an integral over $B_{11} \cap B_{33}^{\infty}$ and $B_{00} \cap B_{33}^{\infty}$. The integrand is the restriction of

$$
I^{(1)}\left(B_{33}^{\infty}\right)=\mathfrak{T}\left(T^{\frac{1}{2}} \mathrm{RN}\left(\varpi_{\epsilon}\right)^{\perp} e^{-T \mathrm{RN}\left(\widetilde{\partial}_{\epsilon}\right)^{\perp 2}}\right)
$$

to each corner. To compute this, refer to the explicit formula (5.7) for $e^{-T R N(\Delta)}$. The integral is at $T=0$, so the only terms that contribute are

$$
\frac{1}{\sqrt{4 \pi T}}\left(e^{-\left|s-s^{\prime}\right|^{2} / 4 T} \mathrm{Id}+e^{-\left|2-s-s^{\prime}\right|^{2} / 4 T} S_{+}+e^{-\left|-2-s-s^{\prime}\right|^{2} / 4 T} S_{-}\right) \text {. }
$$

As noted in section $5, \operatorname{tr} \gamma=\operatorname{tr} \gamma S_{ \pm}=0$. Hence $a_{0,1}$ is also zero.

It follows that $\eta\left(\partial_{\epsilon}\right)$ does have a limit as $\epsilon \rightarrow 0$. As the $(0,0)$ term is constant up to $\epsilon=0$ and all other terms vanish at $\epsilon=0$, the limiting value is

$$
\begin{aligned}
& a_{0,0}=\frac{1}{\sqrt{\pi}} \int_{0}^{\infty} t^{-\frac{1}{2}} d t \int_{\bar{M}} \operatorname{tr}\left(\delta_{\bar{M}} e^{-t \widetilde{\delta}_{\bar{M}}^{2}}\right) \\
& +\frac{1}{\sqrt{\pi}} \int_{0}^{\infty} t^{-\frac{1}{2}} d t \int_{\bar{H}} \operatorname{tr}\left(\check{\partial}_{\bar{H}} e^{-t \widetilde{\partial}_{\bar{H}}^{2}}\right) \\
& +\frac{1}{\sqrt{\pi}} \int_{0}^{\infty} T^{-\frac{1}{2}} d T \int_{-1}^{1} d s \operatorname{tr} \mathfrak{T}\left(\operatorname{RN}\left(\check{\partial}_{\epsilon}\right) e^{-T \operatorname{RN}\left(\widetilde{\jmath}_{\epsilon}\right)^{2}}\right) .
\end{aligned}
$$

The first integral gives $\eta_{b}\left(\check{\bar{M}}_{\bar{M}}\right)$, the second gives zero, since $I^{(1)}\left(B_{33}\right)=0$ and the third is the eta invariant of $\mathrm{RN}(\widetilde{\varnothing})$. Hence we have shown

$$
\lim _{\epsilon \downarrow 0} \eta\left(\check{\partial}_{\epsilon}^{\perp}\right)=\eta_{b}\left(\check{\partial}_{M_{+}}\right)+\eta_{b}\left(\check{\partial}_{M_{-}}\right)+\eta(\mathrm{RN}(ð))
$$

and therefore

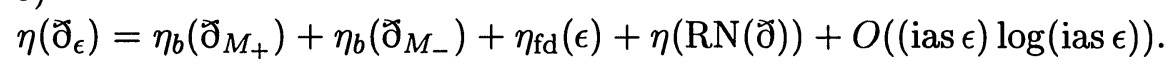


In fact, it is not hard to see that all logarithmic terms $a_{n, 1}, n>0$ vanish. By (2.15), there are no contributions to $a_{n, 1}$ from the faces at finite time becuase they have no terms in their Taylor series at order $n$. So, a logarithmic term can only come from the corners $B_{00}^{\infty} \cap B_{33}^{\infty}$ or $B_{11}^{\infty} \cap B_{33}^{\infty}$. On $B_{00}^{\infty}, \frac{\partial}{\partial t}+\Delta_{\epsilon}$ has the model operator (ias $\epsilon) \frac{\partial}{\partial T}+\Delta_{\bar{M}}$. Hence $H^{(1)}\left(B_{00}^{\infty}\right)$ takes values in $C^{\infty}\left([0, \infty]_{\sqrt{T}} ; \operatorname{Ker} \Delta_{\bar{M}} \otimes \operatorname{Ker} \Delta_{\bar{M}}\right)$ and inductively it follows that

$$
H^{(n)}\left(B_{00}^{\infty}\right) \in C^{\infty}\left([0, \infty]_{\sqrt{T}} ;\left[\operatorname{span} \Phi^{(0)} \ldots \Phi^{(n-1)}\right]^{2}\right),
$$

where the $\Phi^{(i)}$ are defined in section 6.2. Hence, $H^{(n)}$ has, at this corner, an expansion of the form $\sum_{j=0}^{n} \sum_{k=0}^{\infty} c_{j k}(\mathrm{ilg} x)^{-j}(1-s)^{k}$ with only nonnegative powers of $(1-s)$ and only nonpositive powers of (ilg $x)$. As $\operatorname{ex}\left(B_{33}^{\infty}\right)=1$ and $\operatorname{ex}\left(B_{00}^{\infty}\right)=0$, the result follows from (2.15). This improves the error estimate in (9.5) to $O$ (ias $\epsilon)$.

An explicit formula for $\eta(\mathrm{RN}(ð))$ in terms of the scattering matrix is given by Proposition 5.2.

\section{References.}

[1] M.F. Atiyah, V.K. Patodi and I.M. Singer, Spectral asymmetry and Riemannian geometry $I_{2}$ Math. Proc. Camb. Phil. Soc., 77(1975), 43-69.

[2] N. Berline, E. Getzler and M. Vergne, Heat kernels and Dirac Operators, Springer-Verlag 1991.

[3] J.-M. Bismut and J. Cheeger, $\eta$-Invariants and their adiabatic limits, Jour. of the Amer. Math. Soc., 2(1989), 33-70.

[4] J. Brüning and M. Lesch, In preparation.

[5] U. Bunke, $\eta$-invariants for manifolds with boundary, Humboldt SFB 288 preprint no. 52, 1993.

[6] U. Bunke, On the gluing formula for the $\eta$ invariant, Jour. Diff. Geom., 41 (1995) no. 2, 397-448.

[7] Splitting the spectral flow, Humboldt SFB 288 preprint no. 63, 1993.

[8] S.E. Cappell, R. Lee and E.Y. Miller, Self-adjoint operators and manifold decomposition Part I: Low eigenmodes and stretching, preprint, 1993.

[9] J. Cheeger, Spectral Geometry of Singular Riemannian spaces, Jour. Diff. Geom., 18(1983), 575-657. 
[10] , $\eta$-invariants, the adiabatic approximation and conical singularities, Jour. Diff. Geom., 26(1987), 175-221.

[11] X. Dai, Eta invariants for manifolds with boundary, preprint, 1993.

[12] R. D. Douglas and K. P. Wojciechowski, Adiabatic limits of the $\eta$-invariants. The odd dimensional Atiyah-Patodi-Singer problem, Commun. Math. Phys., 142(1991), 139-168.

[13] E. Getzler, A short proof of the Atiyah-Singer index theorem, Topology, 25(1986), 111-117.

[14] A. Hassell, Analytic surgery and analytic torsion, Preprint, 1995.

[15] A. Hassell, R. Mazzeo and R.B. Melrose, A signature theorem on manifolds with corners, Preprint, 1995.

[16] L. Hörmander, The analysis of linear partial differential operators III, Springer Verlag 1985.

[17] L. Ji, Spectral Degeneration of Hyperbolic Riemann Surfaces, J. Diff. Geom., 38(1993), 263-313.

[18] H.B. Lawson Jr. and M.-L. Michelson, Spin Geometry, Princeton Mathematical Series 38, Princeton Univ. Press 1989.

[19] M. Lesch and K.P. Wojciechowski, On the $\eta$-invariant of generalized AtiyahPatodi-Singer boundary value problems, To appear, Ill. Math. J.

[20] R. R. Mazzeo and R. B. Melrose, Analytic surgery and the eta invariant, Geom. Funct. Anal., 5 (1995), no. 1, 14-75.

[21] P. McDonald, The Laplacian on spaces with conic singularities, Thesis, MIT (1990).

[22] R.B. Melrose, The Atiyah-Patodi-Singer index theorem, A. K. Peters 1993.

[23] Differential analysis on manifolds with corners, In preparation.

[24] Calculus of conormal distributions on manifolds with corners, International Mathematics Research Notices 1992 No.3, 51-61. Pseudodifferential Operators, Corners and Singular Limits, Proceedings of the International Congress of Mathematicians (Kyoto, 1990), Math. Soc. Japan 1991, 217-234.

[26] W. Müller, Eta Invariants and Manifolds with Boundary, Jour. Diff. Geom., 40 no. 2(1994), 311-378. 
[27] V.K. Patodi, Curvature and the eigenforms of the Laplace operator, Jour. Diff. Geom., 5(1971), 233-249.

[28] R.T. Seeley and I.M. Singer, Extending $\bar{\partial}$ to singular Riemann surfaces, Jour. Geom. Phys., 5 no. 1(1988), 121-136.

[29] K. P. Wojciechowski, On the additivity of the $\eta$-invariant. The case of a singular tangential operator, Preprint, 1993.

[30] S. Wolpert Spectral limits of hyperbolic surfaces I,II, Invent. Math., 108 (1992), 67-89, 91-129.

MIT

Cambridge, MA 02139

E-MAIL: RBM@MATH.MIT.EDU

AND

STANFORD UNIVERSITY

STANFORD, CA 94305

E-MAIL: MAZZEO@MATH.STANFORD.EDU

AND

E-MAIL: HASSELLA@MATH.STANFORD.EDU

RECeIVEd JANUARY 18Th, 1995. 